\title{
ADAPTATION OF VT-DBR LASERS FOR LIDAR
}

\author{
A Thesis Presented to the \\ Electrical Engineering Department Faculty of \\ California Polytechnic State University, San Luis Obispo \\ In Partial Fulfillment \\ of the Requirements for the \\ Master of Science Degree in Electrical Engineering
}

By

Luke Daniel Horowitz

June 2018 
(C) 2018

Luke Daniel Horowitz

ALL RIGHTS RESERVED 


\section{COMMITTEE MEMBERSHIP}

$\begin{aligned} \text { TITLE: } & \text { Adaptation of VT-DBR Lasers for LIDAR } \\ \text { AUTHOR: } & \text { Luke Daniel Horowitz } \\ \text { DATE SUBMITTED: } & \text { June } 29,2018\end{aligned}$

COMMITTEE CHAIR: Dennis Derickson, Ph.D.

Electrical Engineering Department Chair

COMMITTEE MEMBER:

Chris Wood, Ph.D.

VP Spectroscopy, Insight Photonic Solutions

COMMITTEE MEMBER:

Glen D. Gillen, Ph.D.

Associate Professor of Physics 


\section{ABSTRACT \\ Adaptation of VT-DBR Lasers for LIDAR}

Luke Daniel Horowitz

Vernier Tuned Distributed Bragg Reflector (VT-DBR) lasers have had great success in the field of Swept-Source Optical Coherence Tomography (SS-OCT) due to their continuous and nearly $40 \mathrm{~nm}$ wavelength tuning range in a single longitudinal mode. Fast sweeps allow for real time imaging with micrometer resolution at a distance of a few centimeters. While this laser has proven quite useful as a medical imaging tool via OCT, it has yet to similarly prove itself for general light detection and ranging (LIDAR) applications due to range limitations that arise from a finite laser coherence length. The goal of this thesis is to explore LIDAR applications for VT-DBR lasers and how to improve VT-DBR performance for LIDAR. In the scope of this work, LIDAR is laser imaging at tens or hundreds of meters with a resolution finer than $10 \mathrm{~cm}$. In order to achieve this kind of LIDAR performance with a VT-DBR laser, the laser must have a linewidth less than $1 \mathrm{MHz}$ over a tuning range of around $10 \mathrm{GHz}$. This thesis outlines two methods towards this goal. The bulk of this work is dedicated to looking for and characterizing VT-DBR tuning paths with fundamentally narrow linewidth using microampere currents in both forward and reverse bias conditions. The second part of this thesis is a preliminary design of an optical frequency-locked loop to reduce laser phase noise, which subsequently reduces the laser linewidth.

By tuning with small currents in the forward bias condition, nearly the entire range of laser wavelengths could be tuned to, but areas of narrow linewidth were both sparse and very sensitive to any change in bias. The reverse bias case showed limited but continuous tuning with increased reverse current magnitude. In this reverse biased photo-detector mode the laser exhibited narrower linewidth less than $15 \mathrm{MHz}$, with the linewidth at intrinsically narrow levels when all three sections reverse biased. Also promising was a subset of reverse bias conditions that only used a variable resistance across a laser section with no externally applied bias. This resistance tuning method gave a tuning range of more than $7 \mathrm{GHz}$ while maintaining an intrinsically narrow linewidth.

The optical frequency-locked loop was able to achieve DC frequency locking but unable to reduce laser linewidth. More work needs to be done to achieve enough phase noise reduction to see an appreciable reduction in linewidth. 


\section{ACKNOWLEDGMENTS}

I would first and foremost like to thank Dr. Dennis Derickson for giving me the opportunity to work on this project and for all of the insight he has given me into not only photonics, but also many other far-reaching topics in engineering and life.

I would also like to thank Dr. Chris Wood, Marc Bernard, and all the folks at Insight Photonic Solutions for not only supplying the VT-DBR laser and control PCBA for this project but also giving extremely helpful feedback through the entire process. My time spent as a summer intern at Insight gave me abundant experience as an engineer and was the birthplace of this project.

I would like Dr. Glen Gillen for his continued support of my interest in lasers, optics, and photonics from my undergraduate to my graduate studies. It was his advanced optics course three years ago that first sparked my interest in the field of photonics.

Finally, I would like to thank my family for their continuous love and support throughout my college career. I would not be here without it. 
$\begin{array}{lll}20 & \text { Page }\end{array}$

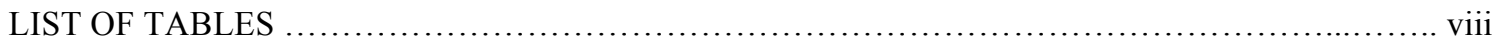

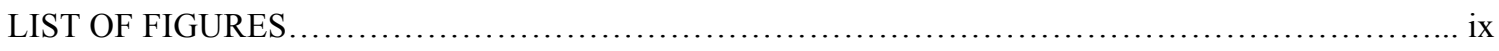

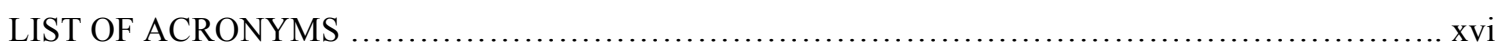

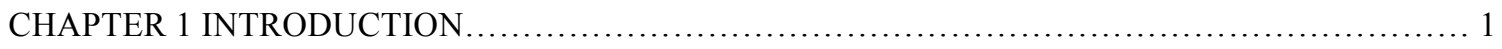

CHAPTER 2 FMCW LIDAR AND SS-OCT BACKGROUND .................................... 3

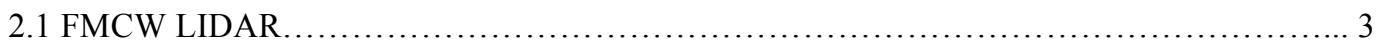

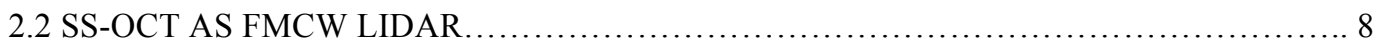

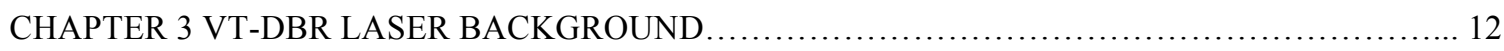

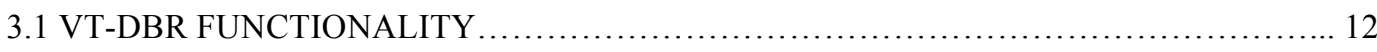

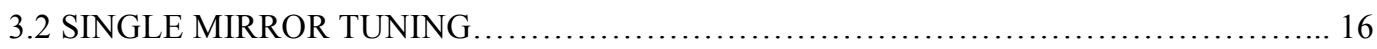

3.3 SIMULTANEOUS TUNING OF FRONT AND BACK MIRRORS ....................... 17

3.4 SIMULTANEOUS TUNING OF FRONT MIRROR, BACK MIRROR, AND PHASE

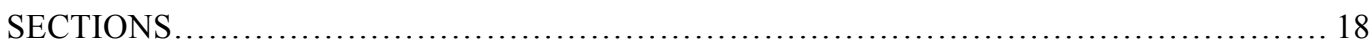

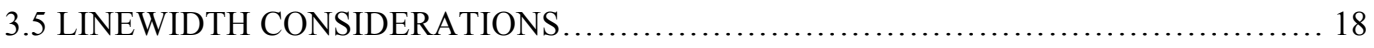

CHAPTER 4 COMMON SOURCES OF NOISE IN OPTICAL MEASURMENTS..................... 21

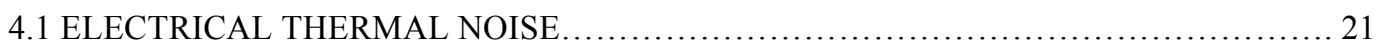

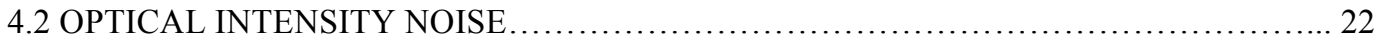

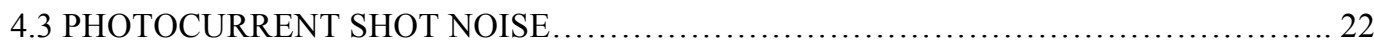

4.4 OPTICAL PHASE NOISE.............................................................. 23

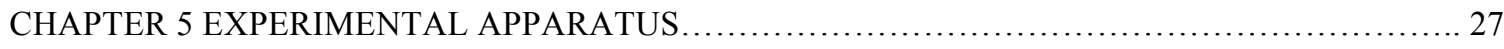

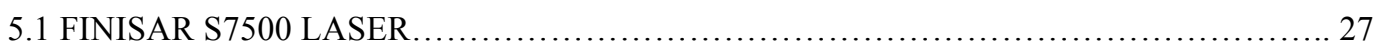

5.2 BREAKOUT BOARD FOR LASER BIASING ................................... 27

5.3 BATTERY-POWERED CURRENT SUPPLIES FOR LOW NOISE BIASING.............. 29

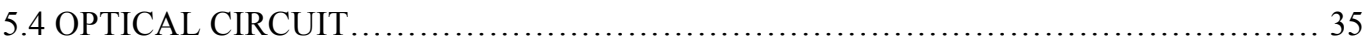

5.5 SELF-HOMODYNE LINEWIDTH ESTIMATION..................................... 36 
CHAPTER 6 BIASING FOR NARROW LINEWIDTH................................... 37

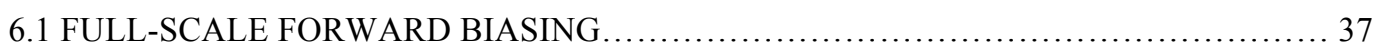

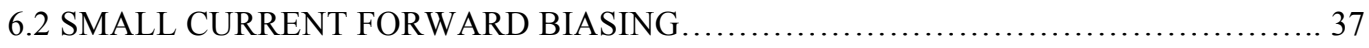

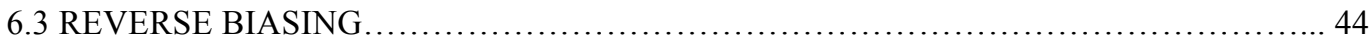

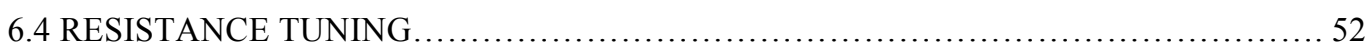

6.5 FREQUENCY RESPONSE COMPARISON OF FORWARD BIASING AND

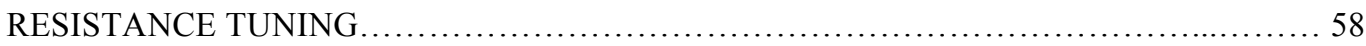

CHAPTER 7 REDUCING LINEWIDTH USING OPTOELECTRONIC FEEDBACK................65

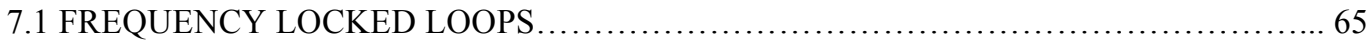

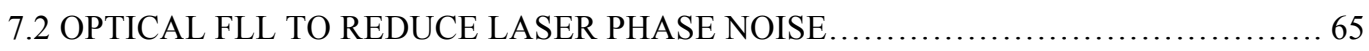

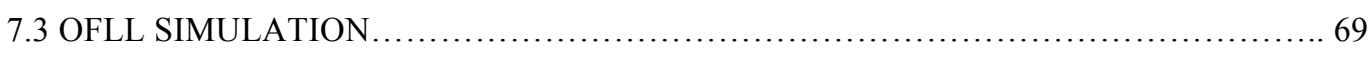

7.4 OPEN LOOP AND CLOSED LOOP PERFORMANCE $\ldots \ldots \ldots \ldots \ldots \ldots \ldots \ldots \ldots \ldots \ldots \ldots . \ldots . \ldots \ldots$

CHAPTER 8 CONCLUSIONS AND FUTURE DIRECTIONS $\ldots \ldots \ldots \ldots \ldots \ldots \ldots \ldots \ldots \ldots \ldots \ldots \ldots \ldots \ldots . \ldots \ldots$

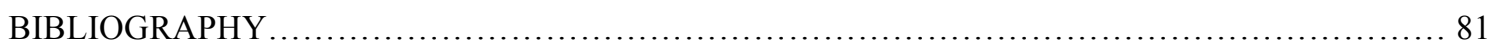




\section{LIST OF TABLES}

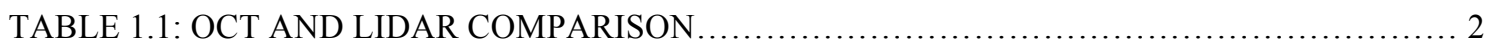

TABLE 8.1: SUMMARY OF LIDAR POTENTIAL FOR EACH MODE OF

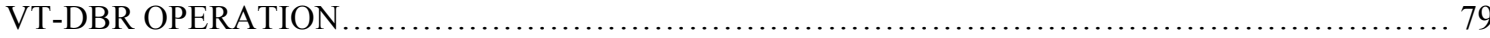


FIGURE 2.2: TRANSMITTED AND RECEIVED FMCW WAVEFORMS DEPICTING VERTICAL DOPPLER SHIFT FROM TARGET SPEED AND HORIZONTAL SHIFT DUE TO TIME DELAY FROM TARGET DISTANCE.

FIGURE 2.3: DEMONSTRATION OF RANGE AMBIGUITY. AT $t=T / 2$, THESE TWO FREQUENCY DIFFERENCES ARE EQUAL, AND $t>T / 2$ GIVES AMBIGUOUS RANGE MEASUREMENTS.

FIGURE 2.4: DEMONSTRATION OF RANGE AMBIGUITY FOR A TIME DELAY $t$ LONGER THAN THE FMCW CHIRP PERIOD $T$.

FIGURE 2.5: OCT CROSS-SECTION OF A HUMAN RETINA (LEFT) AND THE SKIN ON THE PALMAR ASPECT OF THE THUMB (RIGHT). [33]...

FIGURE 2.6: MACROSCOPIC IMAGES SHOWING B-SCAN PATH (LEFT) AND CORRESPONDING CROSS-SECTION (RIGHT) OF THE EPITHELIAL TISSUE IN CANINE CORNEA. [16].

FIGURE 3.1: TOP-DOWN (ABOVE) AND SIDE (BELOW) VIEW OF THE VT-DBR LASER CHIP SHOWING THE VARIOUS SECTIONS OF THE LASER. [9].

FIGURE 3.2: MACROSCOPIC (LEFT) AND MICROSCOPIC (RIGHT) VIEW OF A VT-DBR LASER CHIP TO SHOW SIZE. RIGHTMOST IMAGE SHOWS WIRE-BONDING FOR CURRENT INJECTION INTO EACH SECTION. [18]. 13

FIGURE 3.3: CARRIER DENSITY AS A FUNCTION OF INJECTED CURRENT (LEFT) AND PHOTON DENSITY AS A FUNCTION OF INJECTED CURRENT (RIGHT) FOR A SEMICONDUCTOR LASER. [14].....

FIGURE 3.4: REFLECTIVITY SPECTRUM OF TWO SAMPLED BRAGG REFLECTORS WITH SPACING $\mathrm{L}_{1}>\mathrm{L}_{2}$ AND CORRESPONDING $\Delta \lambda_{1}<\Delta \lambda_{2}$. [4] $\ldots$

FIGURE 3.5: VERNIER EFFECT FOR TWO BRAGG REFLECTORS WITH CLOSE SPACING (TOP). BOTH OF THE PLOTS ON THE BOTTOM LEAD TO STABLE OPERATION, BUT A SLIGHT MISALIGNMENT OF THE REFLECTIVITY PEAKS (BOTTOM RIGHT) WILL LEAD TO DECREASED OUTPUT POWER WITH MORE NARROW LINEWIDTH. [17]

FIGURE 3.6: EXAMPLE TUNING MAP FOR A VT-DBR LASER. THE NUMBER ENCLOSED IN EACH REGION, COLLOQUIALLY CALLED A "LILY-PAD”, IS THE WAVELENGTH IN NANOMETERS. IT IS POSSIBLE TO CONSECUTIVELY TUNE TO EACH WAVELENGTH IN THE TUNING RANGE BY FOLLOWING THESE TUNING PATHS, GIVEN BY COLOR. [9] 16

FIGURE 3.7: SINGLE MIRROR TUNING VIA AN INCREASED FRONT MIRROR CURRENT AND A CONSTANT BACK MIRROR CURRENT. THIS TUNING METHOD LEADS TO LARGE AND DISCONTINUOUS JUMPS IN WAVELENGTH, CALLED "MODE HOPS" GIVEN BY THE INTER-PEAK SPACING IN THE REFLECTIVITY SPECTRUM OF THE MIRROR BEING TUNED. [4]. 
FIGURE 3.8: SIMULTANEOUS MIRROR TUNING VIA A SIMULTANEOUS INCREASE OF FRONT AND BACK MIRROR CURRENT. THIS TUNING METHOD LEADS TO SMALLER BUT STILL DISCONTINUOUS JUMPS IN WAVELENGTH OF APPROXIMATELY 300 PICOMETERS CAUSED BY THE CAVITY MODE SPACING OF THE LASER. [4]....

FIGURE 3.9: SIMULATED (A) AND MEASURED (B) LINEWIDTH FOR VARIOUS FRONT AND BACK MIRROR CURRENTS. NOTE THAT $\mathrm{I}_{\mathrm{FM}}=\mathrm{I}_{\mathrm{BM}}=0 \mathrm{~mA}$ IS IN THE UPPER CORNER OF EACH PLOT. SIMULATION SHOWS A TREND OF LINEWIDTH BROADENING WITH INCREASED CURRENT, WHILE EXPERIMENTAL DATA SHOWS LESS GENERAL LINEWIDTH BROADENING WITH NARROW REGIONS OF MUCH LARGER LINEWIDTH. [20].

FIGURE 3.10: THREE POSSIBLE VT-DBR MODES OF OPERATION.

FIGURE 4.1: (A) SIMPLIFIED DIAGRAM OF A PHOTODIODE CONNECTED TO AN ELECTRICAL AMPLIFIER. (B) THE EQUIVALENT CIRCUIT MODEL INCLUDING THERMAL RESISTIVE NOISE USING IDEAL CURRENT SOURCES.

FIGURE 4.2: CONVERSION OF FREQUENCY MODULATION TO INTENSITY MODULATION FOR AN OPTICAL SIGNAL PASSED THROUGH AN INTERFEROMETER WITH TIME DELAY $T_{O}$. AMPLITUDE OF INTENSITY MODULATION DEPENDS ON SLOPE OF FREQUENCY DISCRIMINATOR AT THE FREQUENCY OF INTEREST. NO CONVERSION HAPPENS AT POINTS OF ZERO SLOPE.

FIGURE 4.3: POWER SPECTRAL DENSITY RESULTING FROM THE CONVERSION OF LASER PHASE-NOISE INTO OPTICAL INTENSITY NOISE FOR BOTH THE COHERENT AND INCOHERENT INTERFERENCE

FIGURE 5.1: BREAKOUT BOARD WITH SMA EDGE-LAUNCH CONNECTIONS 27

FIGURE 5.2: LASER AND BREAKOUT BOARDS ON ALUMINUM HEAT SINK ................. 28

FIGURE 5.3: CIRCUIT DIAGRAM FOR FULL-RANGE BATTERY POWERED

CURRENT SUPPLY

FIGURE 5.4: FULL-RANGE 12V BATTERY POWERED CURRENT SUPPLY WITH SMA CURRENT OUTPUTS. 30

FIGURE 5.5: CIRCUIT DIAGRAM FOR SMALL-CURRENT BATTERY POWERED CURRENT SUPPLY 31

FIGURE 5.6: AA BATTERY POWERED SMALL CURRENT SUPPLY WITH SEMI-RIGID COAXIAL OUTPUT. 31

FIGURE 5.7: CIRCUIT DIAGRAM FOR 9V BATTERY POWERED REVERSE CURRENT SUPPLY USING ML-7905A NEGATIVE VOLTAGE REGULATOR. 32

FIGURE 5.8: 9V BATTERY POWERED REVERSE CURRENT SUPPLY WITH SEMI-RIGID COAXIAL OUTPUT. 33

FIGURE 5.9: VCR CIRCUIT WITH MPF102 JFET_............................................ 34

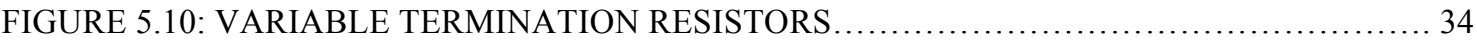

FIGURE 5.11: BLOCK DIAGRAM FOR OPTICAL CIRCUIT SHOWING DIAGNOSTIC SECTION (UPPER RIGHT) AND FEEDBACK SECTION (LOWER LEFT).... 
FIGURE 5.12: BLOCK DIAGRAM FOR SELF-HOMODYNE LINEWIDTH ESTIMATION, WITH CORRESPONDING SPECTRUM AFTER MIXING. [11]....

FIGURE 6.1: SELF-HOMODYNE SIGNAL FOR ALL THREE PASSIVE SECTIONS

FORWARD BIASED. BROAD 112MHz LINEWIDTH CAUSED BY CURRENT

INJECTION INTO BOTH FRONT AND BACK MIRRORS.

FIGURE 6.2: INDEPENDENT SECTION SMALL-CURRENT FORWARD BIAS

WAVELENGTH TUNING. A LARGE PORTION OF THE FULL WAVELENGTH

TUNING RANGE IS COVERED WITH CURRENTS LESS THAN $1 \mathrm{~mA} . .$. 38

FIGURE 6.3: INDEPENDENT SECTION SMALL-CURRENT FORWARD BIAS

LINEWIDTH. TOP PLOT SHOWS THE FULL RANGE OF LINEWIDTH WHILE

THE BOTTOM PLOT SHOWS THE REGIONS OF LINEWIDTH UNDER 100MHz.

IT CAN BE SEEN THAT THERE ARE SOME BIAS CONDITIONS

WITH $>10 \mathrm{MHz}$ LINEWIDTH.

39

FIGURE 6.4: INDEPENDENT SECTION SMALL-CURRENT FORWARD BIAS OUTPUT

POWER VARIATION.

FIGURE 6.5: WAVELENGTH MAP OVER FM AND BM CURRENTS FROM 0 TO 1mA. PHASE SECTION WAS GROUNDED FOR THIS MEASUREMENT. THIS MAP SHOWS LARGE SCALE WAVELENGTH TUNING IS POSSIBLE WITH CURRENTS LESS THAN 1mA

FIGURE 6.6: WAVELENGTH MAP OVER FM AND BM CURRENTS FROM 0 TO 1mA. PHASE CURRENT IS $1 \mathrm{~mA}$ FOR THIS MEASUREMENT. THE EFFECT OF THE ADDITIONAL PHASE CURRENT CAN BE SEEN TO DECREASE THE WAVELENGTH OVER THEN ENTIRE SPACE

FIGURE 6.7: LINEWIDTH MAP OVER FM AND BM CURRENTS FROM 0 TO 1mA. PHASE SECTION IS GROUNDED FOR THIS MEASUREMENT. THERE ARE POCKETS OF NARROW LINEWIDTH BUT A FINER MAPPING OF THIS SPACE IS NEEDED TO DETERMINE THE RANGE OF WAVELENGTHS WITH NARROW LINEWIDTH. .

FIGURE 6.8: LINEWIDTH MAP OVER FM AND BM CURRENTS FROM 0 TO $1 \mathrm{~mA}$. PHASE CURRENT IS 1mA FOR THIS MEASUREMENT. THE ADDITION OF PHASE CURRENT INJECTION IS SEEN TO INCREASE THE LINEWIDTH FOR MOST BIAS CONDITIONS.

FIGURE 6.9: INDEPENDENT SECTION REVERSE BIAS WAVELENGTH TUNING. EACH SECTION EXHIBITS NARROW BUT CONTINUOUS WAVELENGTH TUNING, WITH INCREASED TUNING CAPABILITIES IN THE BACK MIRROR SECTION. 45

FIGURE 6.10: INDEPENDENT SECTION REVERSE BIAS LINEWIDTH. INDEPENDENT FRONT MIRROR TUNING EXHIBITS NARROW LINEWIDTH BUT INDEPENDENT BACK MIRROR TUNING IS SEEN TO EXHIBIT INCREASED LINEWIDTH.

FIGURE 6.11: INDEPENDENT SECTION REVERSE BIAS OUTPUT POWER VARIATION. INCREASED CURRENT MAGNITUDE LEADS TO A DECREASE IN OUTPUT OPTICAL POWER, WITH A GREATER DECREASE IN POWER EXHIBITED BY THE BACK MIRROR SECTION. 
FIGURE 6.12: REVERSE CURRENT WAVELENGTH MAP. PHASE SECTION GROUNDED. REVERSE BIASING OF THE MIRROR SECTIONS LEADS TO A SMOOTH TUNING SURFACE WITH A 40 PICOMETER TUNING RANGE.

FIGURE 6.13: REVERSE CURRENT LINEWIDTH MAP. PHASE SECTION GROUNDED. REVERSE BIASING OF THE MIRROR SECTIONS LEADS TO NARROWER LINEWIDTH COMPARED TO FORWARD BIASING. THIS PLOT SHOWS APPARENT PATHS OF NARROW LINEWIDTH.

FIGURE 6.14: TOP PLOW SHOWS POTENTIAL TUNING PATHS WITH 4-5MHZ LINEWIDTH, WHILE THE BOTTOM PLOT SHOWS THE WAVELENGTH TUNING THAT WOULD ACCOMPANY THESE TUNING PATHS. SOME NARROW LINEWIDTH TUNING PATHS HAVE A LARGER RANGE OF WAVELENGTHS THAN OTHERS.

FIGURE 6.15: REVERSE CURRENT WAVELENGTH MAP. PHASE CURRENT IS -1mA. THE ADDITION OF REVERSE PHASE CURRENT BOTH SHIFTS THE ENTIRE RANGE OF WAVELENGTHS TO THE BLUE, AS WELL AS INCREASE THE MAXIMUM TUNING RANGE GIVEN BY THE FRONT AND BACK MIRRORS ALONE FROM 40 PICOMETERS TO 50 PICOMETERS.

FIGURE 6.16: REVERSE CURRENT LINEWIDTH MAP. PHASE CURRENT IS -1mA. THIS PLOT GIVES THE SURPRISING RESULT THAT REVERSE BIASING ALL THREE SECTIONS CAN LEAD TO LINEWIDTH LESS THAN 5MHZ OVER THE ENTIRE MAP

FIGURE 6.17: CHARACTERISTIC LINESHAPE FOR REVERSE CURRENTS INTO MIRROR SECTIONS AND -1MA PHASE CURRENT. LINEWIDTH IS SEEN TO BE 1.9MHZ. ALL BIAS CONDITIONS SHOWN IN FIGURE 6.15 LEAD TO A LINESHAPE SIMILAR TO THIS ONE.

FIGURE 6.18: RESISTANCE TUNING MECHANISM USES THE VOLTAGE THAT OCCURS ACROSS EACH PASSIVE SECTION AS A SOURCE OF BIAS WITH A VARIABLE RESISTOR TO ADJUST THE CURRENT.

FIGURE 6.19: RELATIONSHIP BETWEEN CURRENT, VOLTAGE, AND WAVELENGTH FOR RESISTANCE TUNING MECHANISM. BACK MIRROR SECTION SHOWN. THE VOLTAGE IS SEEN TO INCREASE FROM ZERO VOLTS TO THE EXPECTED DIODE VOLTAGE DROP OF 0.6 VOLTS, WHILE CURRENT IS SEEN TO DECREASE WITH INCREASED RESISTANCE.

FIGURE 6.20: WAVELENGTH TUNING MAP FOR RESISTANCE TUNING MECHANISM WITH $R_{\text {PHASE }}=0 \Omega$. RESISTANCE TUNING IS SEEN TO EXHIBIT A SMOOTH TUNING SURFACE WITH A TUNING RANGE OF 40 PICOMETERS IN THIS CASE. 54

FIGURE 6.21: WAVELENGTH TUNING MAP FOR RESISTANCE TUNING MECHANISM WITH R RHASE $=10 \mathrm{k} \Omega$. THE ADDITION OF RESISTANCE ACROSS THE PHASE SECTION SHIFTS THE WAVELENGTH TO THE BLUE AND INCREASES THE TUNING RANGE FROM 40 PICOMETERS TO 60 PICOMETERS

FIGURE 6.22: CHARACTERISTIC RESISTANCE TUNING SELF-HOMODYNE SIGNAL WITH LINEWIDTH AROUND 1.8MHz. ALL BIAS CONDITIONS SHOWN IN FIGURES 6.20 AND 6.21 LEAD TO A LINESHAPE SIMILAR TO THIS ONE.

FIGURE 6.23: RESISTANCE TUNING MECHANISM USING A VOLTAGE CONTROLLED RESISTOR COMPRISED OF A JFET IN THE OHMIC REGION OF OPERATION. 
FIGURE 6.24: SIMULATED I-V CHARACTERISTICS FOR MPF102 JFET VCR WITH $\mathrm{R}_{\mathrm{MAX}}=10 \mathrm{k} \Omega$. THIS SIMULATION SHOWS THAT THE VOLTAGE CONTROLLED RESISTOR CAN TAKE ON ANY RESISTANCE BETWEEN ABOUT 700 $\Omega$ AND 10k $\Omega$.

FIGURE 6.25: MEASURED I-V CHARACTERISTICS FOR MPF102 JFET VCR WITH $\mathrm{R}_{\mathrm{MAX}}=10 \mathrm{k} \Omega$ USING A SEMICONDUCTOR PARAMETER ANALYZER.

MEASUREMENT MATCHES WITH SIMULATION.

FIGURE 6.26: VNA MEASUREMENT DIAGRAM. NEED TO DETERMINE $\mathrm{K}_{\text {DISC }}$ AND K $\mathrm{K}_{\text {LASER }}$ FOR THE FORWARD CURRENT INJECTION AND REVERSE BIAS TUNING MECHANISMS. 59

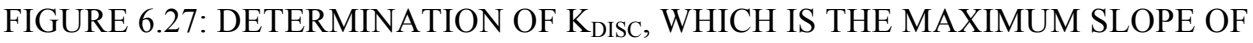
THE PLOT OF DETECTED PHOTOCURRENT VS. FREQUENCY FOR A GIVEN OPTICAL POWER LEVEL. 59

FIGURE 6.28: DETERMINATION OF K $\mathrm{LASER}$ FOR FORWARD CURRENT INJECTION. IT IS FOUND THAT $\mathrm{K}_{\mathrm{LASER}}=-85 \mathrm{GHz} / \mathrm{mA}$ FOR FORWARD CURRENT INJECTION . 60

FIGURE 6.29: DETERMINATION OF K KASER FOR REVERSE CURRENT REVERSE BIASING. IT IS FOUND THAT $\mathrm{K}_{\mathrm{LASER}}=11 \mathrm{GHz} / \mathrm{mA}$ FOR REVERSE BIASING. 60

FIGURE 6.30: LTSPICE SIMULATION SCHEMATIC FOR DIRECT INJECTION (TOP) AND JFET TUNING (BOTTOM). IN BOTH CASES, THE LASER PHASE SECTION IS MODELED AS A VOLTAGE SOURCE AND THE BALANCED PHOTO-DETECTOR IS MODELED AS TWO DEPENDENT CURRENT SOURCES WITH EQUAL AND OPPOSITE CURRENTS GIVEN BY $\mathrm{K}_{\mathrm{DISC}} * \mathrm{~K}_{\mathrm{LASER}} * \mathrm{I}_{\mathrm{PHASE}}$....

FIGURE 6.31: SIMULATED FREQUENCY RESPONSE FOR DIRECT INJECTION TUNING, SHOWN IN THE TOP PLOT OF FIGURE 6.30. THE SOLID LINE SHOWS THE SIGNAL MAGNITUDE WHILE THE DOTTED LINE SHOWS THAT PHASE SHIFT. 62

FIGURE 6.32: SIMULATED FREQUENCY RESPONSE FOR JFET RESISTANCE TUNING, SHOWN IN THE BOTTOM PLOT OF FIGURE 6.30. THE SOLID LINE SHOWS THE SIGNAL MAGNITUDE WHILE THE DOTTED LINE SHOWS THAT PHASE SHIFT. 62

FIGURE 6.33: MEASURED FREQUENCY RESPONSE FOR DIRECT CURRENT INJECTION TUNING MECHANISM. DC GAIN IS SEEN TO BE ABOVE 20dB, WHICH MATCHES THE CORRESPONDING SIMULATION SHOWN IN FIGURE 6.31. DIPS IN SIGNAL MAGNITUDE OCCUR ROUGHLY EVERY 50MHz, BUT THIS EFFECTIS NOT FULLY UNDERSTOOD.

FIGURE 6.34: MEASURED FREQUENCY RESPONSE FOR JFET RESISTANCE TUNING MECHANISM. DC GAIN IS SEEN TO BE LESS THAN -20dB, WHICH MATCHES THE CORRESPONDING SIMULATION SHOWN IN FIGURE 6.31. THE SIGNAL MAGNITUDE RISES MORE THAN 10DB IN THE FIRST 250MHZ AND DIPS IN SIGNAL MAGNITUDE OCCUR ROUGHLY EVERY 50MHz, BUT THESE EFFECTS ARE NOT FULLY UNDERSTOOD. ... 64

FIGURE 7.1: BASIC BLOCK DIAGRAM OF A FLL SHOWING THE MAIN COMPONENTS: A FREQUENCY DISCRIMINATOR, A LOOP FILTER, AND A VOLTAGE-CONTROLLED OSCILLATOR. 
FIGURE 7.2: BASIC BLOCK DIAGRAM OF AN OFLL. THE FREQUENCY DISCRIMINATOR IS A FIBER OPTIC INTERFEROMETER AND BALANCED PHOTO-DETECTOR, THE LOOP FILTER IS AN OP-AMP INTEGRATOR, AND THE VCO IS THE LASER.

FIGURE 7.3: BLOCK DIAGRAM OF EXPERIMENTAL OFLL WITH DIRECT CURRENT INJECTION INTO THE PHASE SECTION THROUGH A $100 \mathrm{k} \Omega$ RESISTOR. THE INTEGRATOR WAS REALIZED USING AN ADA4817 HIGH-SPEED OP-AMP.

FIGURE 7.4: BLOCK DIAGRAM OF EXPERIMENTAL OFLL WITH MPF102 JFET DRIVER ACTING AS A VOLTAGE-CONTROLLED RESISTOR PERFORMING RESISTANCE TUNING ON THE LASER PHASE SECTION.

FIGURE 7.5: DETERMINATION OF OFLL SIGNAL-TO-NOISE RATIO, GIVEN BY THE DIFFERENCE IN dB OF THE FM + AM SIGNAL AND THE AM ONLY SIGNAL. BOTH THE AM ONLY AND FM + AM SIGNALS ARE WELL ABOVE THE INSTRUMENT NOISE FLOOR. 68

FIGURE 7.6: FM TO AM CONVERSION AT TWO DIFFERENT BIAS CONDITIONS SHOWING VARIATION IN FM NOISE SPECTRUM. BOTH PLOTS HAD $\mathrm{I}_{\mathrm{GAIN}} \approx 120 \mathrm{~mA}$ AND $\mathrm{I}_{\mathrm{SOA}} \approx 30 \mathrm{~mA}$. TOP PLOT WAS MEASURED WITH $\mathrm{R}_{\mathrm{FM}}=\mathrm{R}_{\mathrm{BM}}=0 \Omega$ AND THE BOTTOM PLOT WAS MEASURED WITH $\mathrm{R}_{\mathrm{FM}}=0 \Omega \quad$ AND $\mathrm{R}_{\mathrm{BM}} \approx 5 \mathrm{k} \Omega$ 69

FIGURE 7.7: SIMULATED ADA4817 INTEGRATOR SCHEMATIC (TOP) AND FREQUENCY RESPONSE (BOTTOM). THE INTEGRATOR INPUT IS AN AC VOLTAGE SOURCE V4 AND THE OUTPUT IS THE VOLTAGE ACROSS R4. THE SOLID LINE SHOWS THE SIGNAL MAGNITUDE AND THE DASHED LINE SHOWS THE PHASE SHIFT

FIGURE 7.8: OPEN LOOP LTSPICE SCHEMATIC OF DIRECT CURRENT INJECTION OFLL. THIS SIMULATION BUILDS UPON THE SIMULATION SHOWN IN THE TOP PLOT OF FIGURE 6.30 BY ROUTING THE ERROR SIGNAL OUT OF THE TRANSIMPEDENCE AMPLIFIER THROUGH THE ADA4817 INTEGRATOR AND A LOSSLESS 50 $\Omega$ TRANSMISSION LINE WITH A TIME DELAY GIVEN BY THE OVERALL DELAY IN THE LOOP.

FIGURE 7.9: SIMULATED OPEN LOOP FREQUENCY RESPONSE OF OFLL TUNED VIA DIRECT CURRENT INJECTION. THE SOLID LINE SHOWS THE SIGNAL MAGNITUDE AND THE DASHED LINE SHOWS THE PHASE SHIFT. THE SIGNAL AT THE OUTPUT OF THE TRANSMISSION LINE IS GIVEN BY V(feedbackdelay) AND CAN BE SEEN TO HAVE A 75dB GAIN AT DC AND A -3dB BANDWIDTH AROUND 10kHz. PHASE MARGIN IS AROUND 70 AT ROUGHLY 15MHz.

FIGURE 7.10: OPEN LOOP LTSPICE SCHEMATIC OF JFET DRIVEN OFLL. THIS SIMULATION BUILDS UPON THE SIMULATION SHOWN IN THE BOTTOM PLOT OF FIGURE 6.30 BY ROUTING THE ERROR SIGNAL OUT OF THE TRANSIMPEDENCE AMPLIFIER THROUGH THE ADA4817 INTEGRATOR AND A LOSSLESS $50 \Omega$ TRANSMISSION LINE WITH A TIME DELAY GIVEN BY THE OVERALL DELAY IN THE LOOP.... 
FIGURE 7.11: SIMULATED OPEN LOOP FREQUENCY RESPONSE OF JFET DRIVEN OFLL. THE SOLID LINE SHOWS THE SIGNAL MAGNITUDE AND THE DASHED LINE SHOWS THE PHASE SHIFT. THE SIGNAL AT THE OUTPUT OF THE TRANSMISSION LINE IS GIVEN BY V(feedbackdelay) AND CAN BE SEEN TO HAVE A 20dB GAIN AT DC AND A -3dB BANDWIDTH AROUND 10kHz. PHASE MARGIN IS AROUND $75^{\circ}$ AT ROUGHLY $17 \mathrm{MHz}$.

FIGURE 7.12: RESISTANCE TUNING OF THE MIRROR SECTIONS WITH FEEDBACK DISABLED. EACH SECTION SHOWS A WAVELENGTH TUNING RANGE OF ABOUT $30 \mathrm{pm}$. IN THIS MEASUREMENT $\mathrm{I}_{\mathrm{GAIN}} \approx 120 \mathrm{~mA}, \mathrm{I}_{\mathrm{SOA}} \approx 30 \mathrm{~mA}$, AND THE UNUSED PASSIVE SECTIONS WERE SHORTED TO GROUND.

FIGURE 7.13: SELF-HOMODYNE SIGNAL SHOWS LASER LINEWIDTH WITH FEEDBACK DISABLED AROUND 7MHZ. IN THIS MEASUREMENT I $\mathrm{I}_{\mathrm{GAIN}} \approx 120 \mathrm{MA}$, $\mathrm{I}_{\mathrm{SOA}} \approx 30 \mathrm{MA}, \mathrm{R}_{\mathrm{FM}}=0 \Omega, \mathrm{AND} \mathrm{R}_{\mathrm{BM}}=10 \mathrm{~K} \Omega$.

FIGURE 7.14: RESISTANCE TUNING OF THE MIRROR SECTIONS WITH FEEDBACK ENABLED IN THE DIRECT CURRENT INJECTION CASE. IN THIS MEASUREMENT $\mathrm{I}_{\mathrm{GAIN}} \approx 120 \mathrm{~mA}$ AND $\mathrm{I}_{\mathrm{SOA}} \approx 30 \mathrm{~mA}$. THE UNUSED MIRROR SECTION IN EACH MEASUREMENT WAS SHORTED TO GROUND. WHEN THE LOOP IS LOCKED, THERE IS NO CHANGE IN LASER WAVELENGTH FOR A CHANGE IN BIAS CONDITIONS WITHIN THE LOCKING RANGE OF THE LOOP. DIRECT CURRENT INJECTION OFLL IS SEEN TO HAVE A MORE ROBUST LOCKING CAPABILITY OVER A WIDER RANGE OF TUNING RESISTANCE. 75

FIGURE 7.15: RESISTANCE TUNING OF THE MIRROR SECTIONS WITH FEEDBACK ENABLED IN THE JFET DRIVEN CASE. IN THIS MEASUREMENT I $\mathrm{I}_{\mathrm{GAIN}} \approx 120 \mathrm{MA}$ AND $\mathrm{I}_{\mathrm{SOA}} \approx 30 \mathrm{MA}$. THE UNUSED MIRROR SECTION IN EACH MEASUREMENT WAS SHORTED TO GROUND. WHEN THE LOOP IS LOCKED, THERE IS NO CHANGE IN LASER WAVELENGTH FOR A CHANGE IN BIAS CONDITIONS WITHIN THE LOCKING RANGE OF THE LOOP. SMALLER LOCKING RANGE IN THE JFET DRIVEN OFLL SHOWS A LESS ROBUST DC LOCK COMPARED TO THE DIRECT CURRENT INJECTION CASE.

FIGURE 7.16: SELF-HOMODYNE SIGNAL FOR OFLL WITH DIRECT CURRENT INJECTION WITH FEEDBACK ENABLED SHOWS LASER LINEWIDTH AROUND $7 \mathrm{MHz}$. IN THIS MEASUREMENT $\mathrm{I}_{\mathrm{GAIN}} \approx 120 \mathrm{~mA}, \mathrm{I}_{\mathrm{SOA}} \approx 30 \mathrm{~mA}, \mathrm{R}_{\mathrm{FM}}=0 \Omega$, AND $\mathrm{R}_{\mathrm{BM}}=10 \mathrm{k} \Omega$. IN THIS CASE, THE MIRROR SECTIONS ARE UNDER REVERSE BIAS WHILE THE PHASE SECTION IS UNDER FORWARD BIAS.

FIGURE 7.17: SELF-HOMODYNE SIGNAL FOR OFLL WITH JFET DRIVER WITH FEEDBACK ENABLED SHOWS LASER LINEWIDTH AROUND 10MHZ. IN THIS MEASUREMENT $\mathrm{I}_{\mathrm{GAIN}} \approx 120 \mathrm{MA}, \mathrm{I}_{\mathrm{SOA}} \approx 30 \mathrm{MA}, \mathrm{R}_{\mathrm{FM}}=0 \Omega$, AND $\mathrm{R}_{\mathrm{BM}}=10 \mathrm{~K} \Omega$. IN THIS CASE, THE PHASE SECTION AND MIRROR SECTIONS ARE ALL UNDER REVERSE BIAS. 


\section{LIST OF ACRONYMS}

VT-DBR: Vernier Tuned Distributed Bragg Reflector

SS-OCT: Swept-Source Optical Coherence Tomography

FMCW: Frequency-Modulated Continuous Wave

LIDAR: Light Detection and Ranging

FLL: Frequency-Locked Loop

OFLL: Optical Frequency-Locked Loop

FM: Front Mirror section

BM: Back Mirror section

SOA: Semiconductor Optical Amplifier

RIN: Relative Intensity Noise

JFET: Junction Field-Effect Transistor

VCR: Voltage-Controlled Resistor

AM: Amplitude Modulation

FM: Frequency Modulation 


\section{Chapter 1: Introduction}

Vernier Tuned Distributed Bragg Reflector (VT-DBR) lasers have led to great advances in the field of Swept-Source Optical Coherence Tomography (SS-OCT) for a number of reasons. The infrared laser's monolithic semiconductor construction leads to an akinetic structure that allows for high speed tuning, while the VT-DBR topology tuned via the Vernier Effect allows for a nearly 40 nanometer wavelength tuning range in a single longitudinal mode. These fast sweeps allow for real time 3D imaging of structures with micrometer resolution at a distance of a few centimeters. This range and resolution has proven to be ideal for a number of in-vivo medical imaging applications $[4,9,16,26,31,32,33,34]$.

While this is laser has proven quite useful as a medical imaging tool via OCT, it has yet to similarly prove itself for general light detection and ranging (LIDAR) applications due to range limitations that arise from a finite laser coherence length via a non-zero laser linewidth. [31] Centimeter scale range has not been an issue for most OCT applications, so laser linewidth has usually been neglected in favor of an increased side-mode suppression ratio (SMSR), which is more important for OCT imaging. In frequency modulated continuous wave (FMCW) LIDAR, of which SS-OCT is a subset, increased linewidth leads to a degradation of signal-to-noise ratio (SNR) on the LIDAR return signal. This reduction of SNR leads to a limitation on the maximum usable range for a given receiver system with its own characteristic noise floor. VT-DBR lasers typically exhibit intrinsic linewidth around $2 \mathrm{MHz}$, but the linewidth can be as wide as 100s of $\mathrm{MHz}$ depending on the combination of front reflector, back reflector, and phase section currents.

In order to open the application space for VT-DBR lasers in LIDAR, the working range must be increased. An improvement of range follows directly from a reduction in laser linewidth. One methodology would be to only tune the laser around points of narrow linewidth, which sacrifices range resolution due to sweeping over a smaller bandwidth, but maintains a relatively simple LIDAR system. The other methodology is to add electro-optical feedback circuitry to reduce linewidth, which leads to improved range and range resolution over topologies with no feedback, at the expense of increased complexity in the LIDAR system.

Table 1.1 summarizes the difference in requirements for OCT imaging and LIDAR. It shows stark differences in performance that indicate a new mode of VT-DBR operation is needed to create a successful LIDAR system. 


\begin{tabular}{|lll|}
\hline Parameter & Swept-Source OCT & FMCW LIDAR \\
\hline Range Resolution & $30 \mu \mathrm{m}$ & $1.5 \mathrm{~cm}$ \\
\hline Frequency Tuning Bandwidth & $5 \mathrm{THz}$ & $10 \mathrm{GHz}$ \\
\hline Imaging Distance & $2 \mathrm{~cm}$ & $5-100+\mathrm{m}$ \\
\hline Laser Linewidth & $<1 \mathrm{GHz}$ & $<1 \mathrm{MHz}$ \\
\hline Repetition Rate & $200 \mathrm{kHz}$ & $200 \mathrm{kHz}-1 \mathrm{MHz}$ \\
\hline
\end{tabular}

Table 1.1: OCT and LIDAR comparison

This work aims to explore both of the possible methods for narrow linewidth. First, a comparison of three possible narrow linewidth tuning schemes is performed. The first tuning scheme is with microampere scale currents being injected into the passive sections to avoid linewidth broadening due to increased shot noise. The second is to operate each passive laser section as a photo-detector, with a reverse bias drawing current out of the section. The third tuning scheme is to remove the external bias on the passive sections and only have a variable resistance connecting each section to laser ground. This uses the diode voltage across each section as a bias source to draw current from the laser. Finally, a preliminary design of an Optical Frequency-Locked Loop to reduce laser phase noise is introduced. An electro-optical feedback mechanism is added via negative feedback from an asymmetric Mach-Zehnder (MZ) frequency discriminator passed through a loop-filter and fed back into the VT-DBR phase section. 


\section{Chapter 2: FMCW LIDAR and SS-OCT Background}

\subsection{FMCW LIDAR}

Light Detection and Ranging, or LIDAR, is a high-resolution imaging technique that uses laser light to determine the range, velocity, and shape of a remote object. LIDAR has been used in a range of scientific, commercial, and military applications. Scientific LIDAR applications usually fall into the realms of topography, geology, or meteorology, where the LIDAR system is used to map terrain and geological structures or measure atmospheric processes like vortex flow or cloud formation [28, 29]. Commercial applications of LIDAR most often come in the form of vibrometry or computer vision for autonomous vehicles or industrial robotics $[5,6,13]$. Some military applications of LIDAR, often referred to as LADAR, are imaging for unmanned air vehicles (UAVs) or target recognition for weapon systems [7]. Regardless of the application, the characteristics of the LIDAR target like range, speed, and shape are determined through measurement of a phase delay, frequency change, or time delay between transmitted and received waveforms.

In frequency modulated continuous wave (FMCW) LIDAR systems, the target range determines the frequency difference between a transmitted and received chirp waveform. The transmitted chirp waveform is usually a triangle or saw-tooth ramp of frequency over time. Figure 2.1 shows a general

FMCW LIDAR system block diagram.

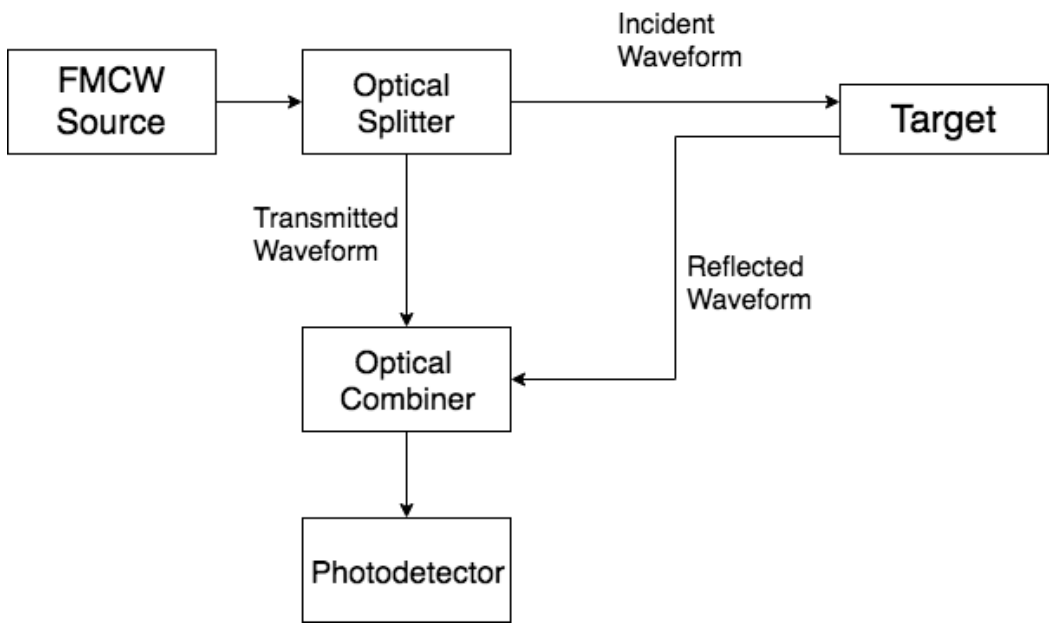

Figure 2.1: General FMCW LIDAR block diagram. 
The FMCW optical signal is split equally into two paths. The incident waveform is reflected off of a target and combined with the original transmitted FMCW signal using an optical combiner. The combined signal is then detected using a photo-detector, whose output is then analyzed in the time and frequency domains. Due to bandwidth limits of the photo-detector, only the down-mixed or difference frequency of the two optical signals will be present at the output. This system is simply a swept-source interferometer with an unknown path length difference in one interferometer arm. The purpose of the system is to determine this path length difference.

The transmitted and received waveforms are shown in Figure 2.2. The changes in the received signal are caused by reflection of the original transmitted signal off of a distant object, and will contain a vertical Doppler shift due to the object's speed and a horizontal shift due to the round-trip time delay caused by distance to the object.

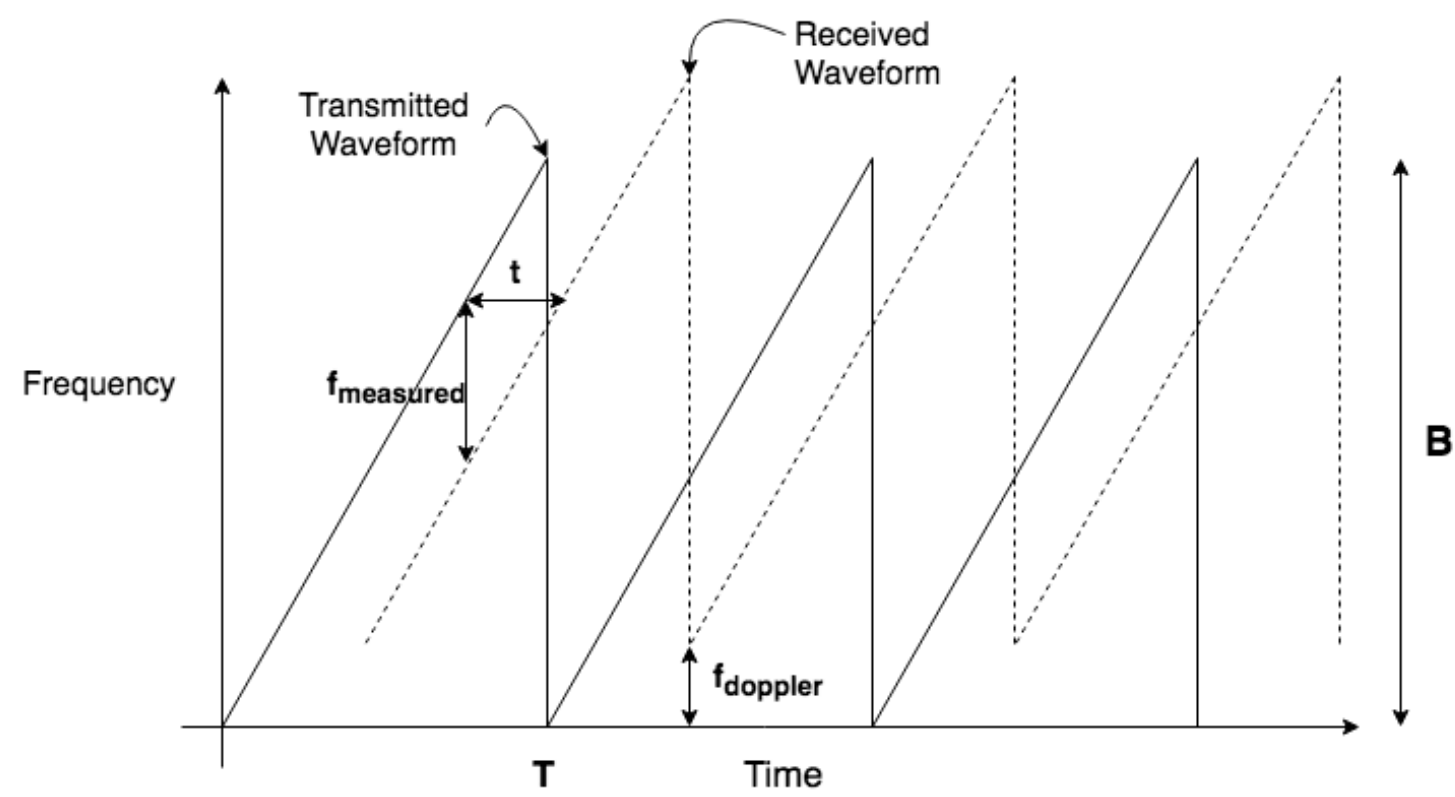

Figure 2.2: Transmitted and received FMCW waveforms depicting vertical Doppler shift from target speed and horizontal shift due to time delay from target distance.

Useful information about the target can be determined using a few simple physical relationships.

First, the range to the target can be determined via the equation

$$
v_{p}=\frac{2 R}{t}
$$

where $v_{p}$ is the velocity of propagation, $t$ is the round trip delay, and $R$ is the range to the target. The velocity of propagation is a known constant of the system, and is equal to the speed of light divided by the 
index of refraction of the medium through which the signal was transmitted. If we assume that the object is stationary, there is no Doppler shift and the time delay is simply the measured frequency difference divided by the slope of the FMCW waveform. This relationship is shown via the equation

$$
t=\frac{f_{\text {measured }}}{\left(\frac{B}{T}\right)}
$$

in which $f_{\text {measured }}$ is the measured frequency difference between the transmitted and received waveform, $B$ is the FMCW chirp bandwidth, $T$ is the FMCW chirp period, and $B / T$ is the slope of the FMCW waveform. By combining Eq. 2.1 and 2.2, the range $R$ can be solved for as

$$
R=\frac{v_{p}}{2} \frac{f_{\text {measured }}}{\left(\frac{B}{T}\right)}
$$

If the target is not stationary, but is traveling with some velocity, the received waveform will experience a vertical shift via the Doppler effect. The magnitude and sign of the Doppler shift is determined by the speed and direction of travel. Higher speeds will lead to a shift of a larger magnitude, and the frequency of the reflected waveform will either increase if the target is moving towards the transmitter or decrease if the object is moving away from the transmitter. The Doppler shift is given by the equation

$$
f_{\text {doppler }}=\frac{2\left(\overrightarrow{v_{r}}-\overrightarrow{v_{s}}\right)}{v_{p}} f_{o}=\frac{2 \Delta v}{v_{p}} f_{o} \cos \theta
$$

or equivalently rearranging for the relative velocity along the line of sight,

$$
\Delta v=\frac{f_{\text {doppler }}}{f_{o}} \frac{v_{p}}{2 \cos \theta}
$$

In Eq. 2.4, $f_{\text {doppler }}$ is the frequency shift due to the Doppler effect, $v_{r}$ is the relative velocity of the receiver in the medium, $v_{s}$ is the relative velocity of the source or target in the medium, $\Delta v$ is the relative velocity between the target and the system, $\theta$ is the angle measured between the target velocity vector and the LIDAR line of sight., and $f_{o}$ is the frequency of the optical output which is a function of time due to the FMCW waveform. The value $\Delta v$ is the radial velocity, or the relative velocity along a straight line connecting the transmitter and target. [10] Measuring the Doppler shift directly is more complicated than measuring the difference frequency, $f_{\text {measured }}$, but since the difference frequency has information about both 
the target range and velocity, it is difficult to determine either the range or speed of the target with the measured frequency difference alone.

The configuration of the FMCW chirp plays an important role in the LIDAR system performance. First, the period of the FMCW ramp, $T$, limits the maximum detection range. Equation 2.1 shows that the time delay, $t$, is dependent on the range to the target. For an unambiguous range measurement, the time delay $t$ must be less than half the FMCW chirp period $T$. The time delay must be less than half the FMCW chirp period because of the multiple difference frequencies that are detected using the FMCW topology. This ambiguity is shown in Figures 2.3 and 2.4 for the case of a stationary target, and the maximum detection range as a function of FMCW chirp period is given in Equation 2.5. Equation 2.5 was found by combining Equations 2.2 and 2.3 with $t=T / 2$.

$$
R_{\max }=\frac{v_{p} T}{4}
$$

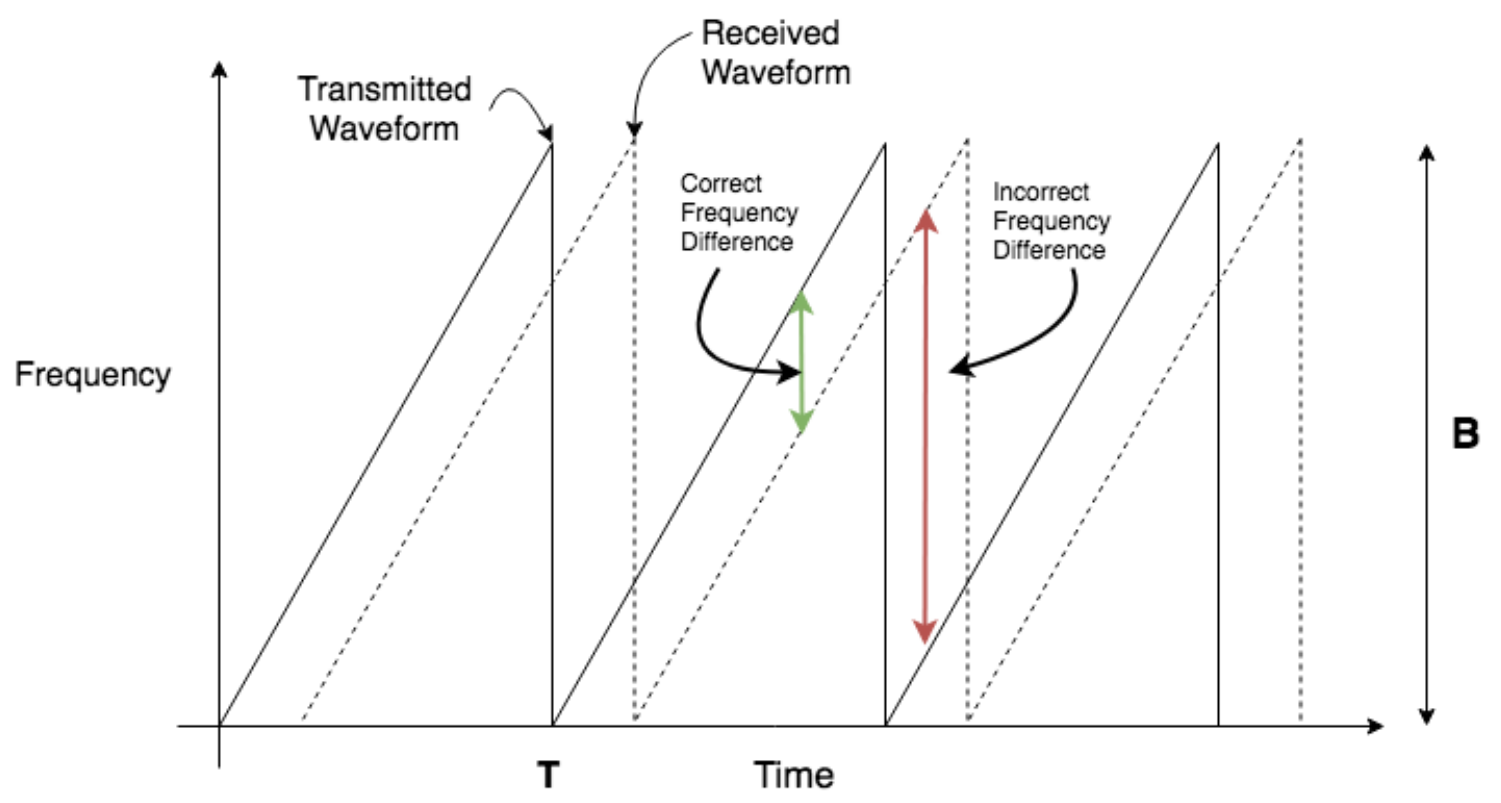

Figure 2.3: Demonstration of range ambiguity. At $t=T / 2$, these two frequency differences are equal, and $t>T / 2$ gives ambiguous range measurements. 


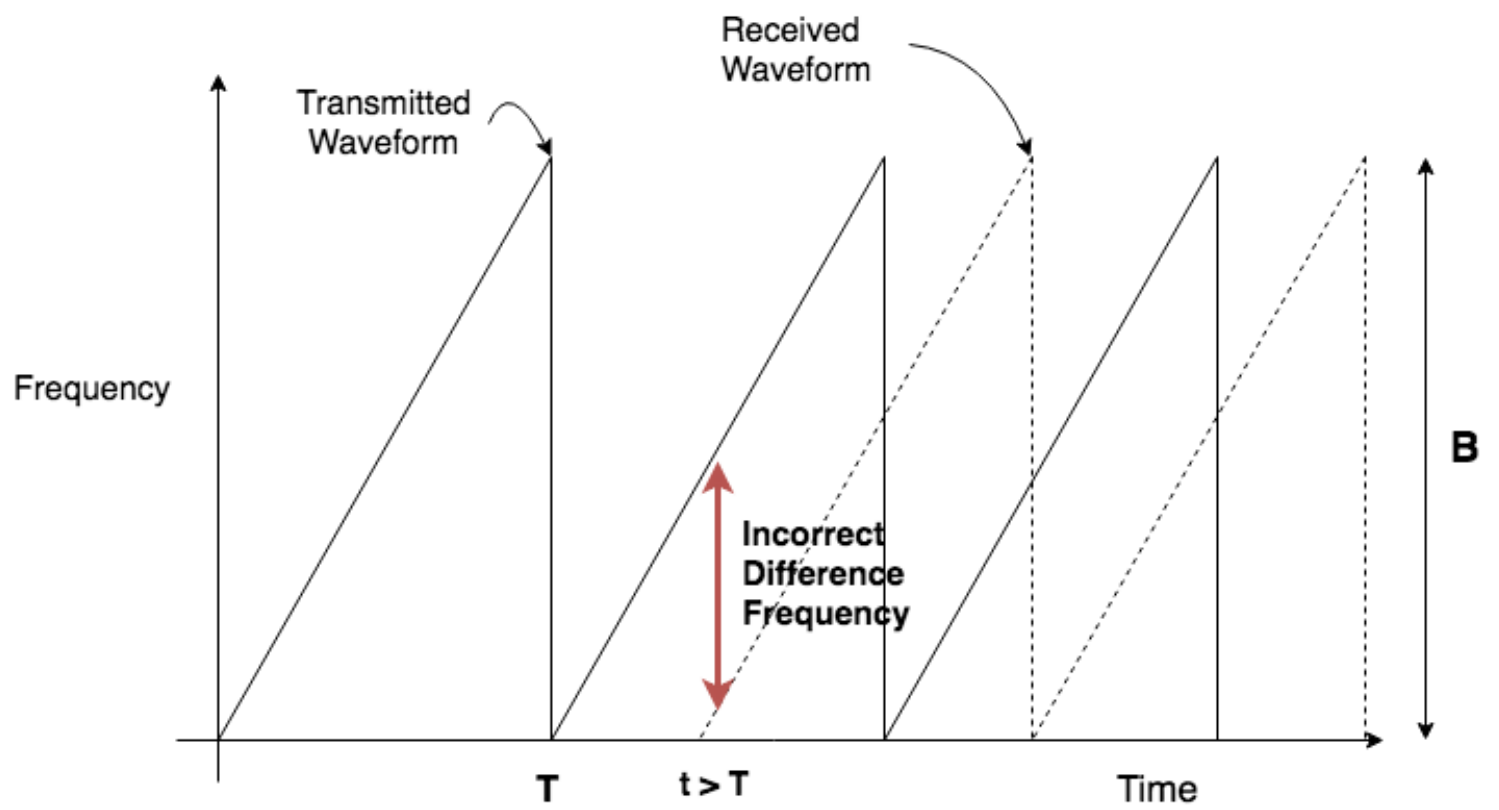

Figure 2.4: Demonstration of range ambiguity for a time delay $t$ longer than the FMCW chirp period $T$.

An obvious conclusion can be drawn from Equation 2.5: the maximum detection range can be extended by an increased FMCW chirp period. Less obvious, however, is the tradeoff between maximum detection range and the sensitivity of the difference frequency with respect to object distance. For a large detection range given by a longer chirp period, the change in measured difference frequency will be very small. This can be seen by rearranging Equation 2.3 to solve for the measured frequency:

$$
f_{\text {measured }}=\frac{2 R}{v_{p}}\left(\frac{B}{T}\right)
$$

Equation 2.6 shows that the measured frequency difference is inversely proportional to the FMCW chirp period. This implies that using a laser with a wide linewidth in a system with a large $T$ may lead to changes in frequency difference that cannot be resolved. The converse is also true: systems with a short chirp period $T$ will be very sensitive to small changes in object distance. Thus linewidth is not an important consideration for systems with small detection range, due to the large changes in measured difference frequency that arise from a change in object distance.

Another important system parameter is the chirp bandwidth B, or the range of frequencies over which the FMCW chirp waveform is swept. The bandwidth of the chirp determines the precision of the 
range measurement, or equivalently the range resolution. The range resolution of the system is given in Equation 2.7:

$$
\delta R=\frac{v_{p}}{2 B}
$$

Equation 2.7 shows that the range resolution is inversely proportional to the chirp bandwidth, which means that the range resolution is smaller for larger chirp bandwidths. Smaller range resolution implies a more precise measurement of the target's range; so large chirp bandwidths are a desirable characteristic of FMCW LIDAR systems. Practically speaking, the chirp bandwidth is limited because the maximum difference frequency must be less than the maximum input frequency of the detector. Thus, both the maximum range and minimum range resolution are constrained by properties of the FMCW source and detection method.

\subsection{SS-OCT as FMCW LIDAR}

LIDAR is also used in medical applications to image below the surface of biological tissues.

Because microwaves and millimeter waves do not penetrate biological tissues, optical frequencies must be used for this imaging scheme. This short range LIDAR technique is commonly known as Optical Coherence Tomography (OCT), and allows biological samples to be imaged at varying depths. Imaging is performed via interferometry to obtain distance information from time-delayed signals. Due to the noninvasive properties of this technique, it is often used for in-vivo imaging of sensitive or hard-to-reach areas in biological systems. Examples of this include imaging the outer structure of the eye or imaging a few millimeters below the surface of biological tissues like skin or arterial walls [16, 33]. This technique could, in principle, image any semi-transparent organic material to a depth of a few millimeters. Figure 2.5 shows an example of OCT cross-sections of a human retina (left) and the skin on the palmar aspect of the thumb (right). Images like the ones in Figure 2.5 allow medical experts to analyze small biological structures and look for potential issues. 

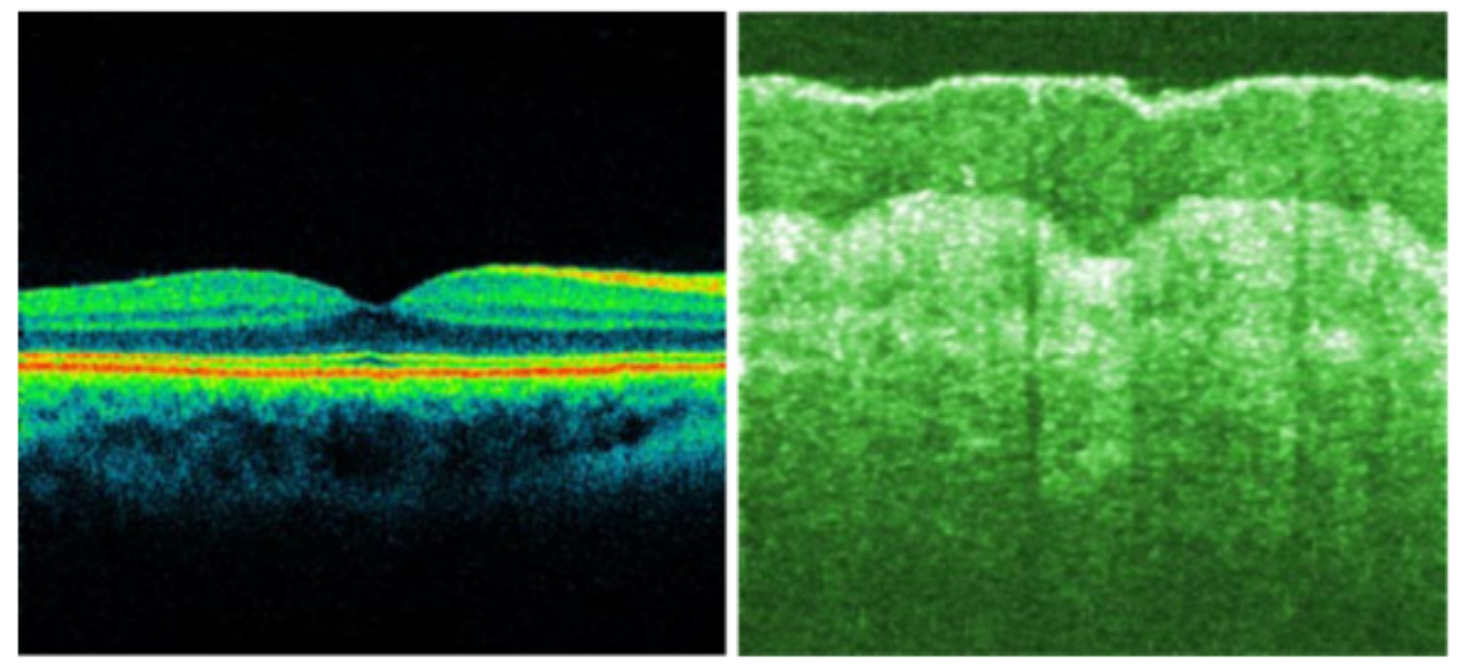

Figure 2.5: OCT cross-section of a human retina (left) and the skin on the palmar aspect of the thumb (right). [33]

OCT allows for imaging in one, two, or three dimensions. For a stationary OCT system, a onedimensional scan is performed on a single point. This is called an axial scan (A-Scan), and reveals a depth profile at the point of the scan. By using a scanning mechanism to move the imaging system in a transverse direction, a cross-sectional image of the structure is revealed, called a B-Scan. In the same manner, many B-scans can be performed as the system moves perpendicular to the B-scan direction, which gives a threedimensional mapping of the structure of interest. This three-dimensional mapping is called a C-Scan. [33] Figure 2.6 shows some examples of B-Scans and their corresponding cross-sections performed on canine cornea. 

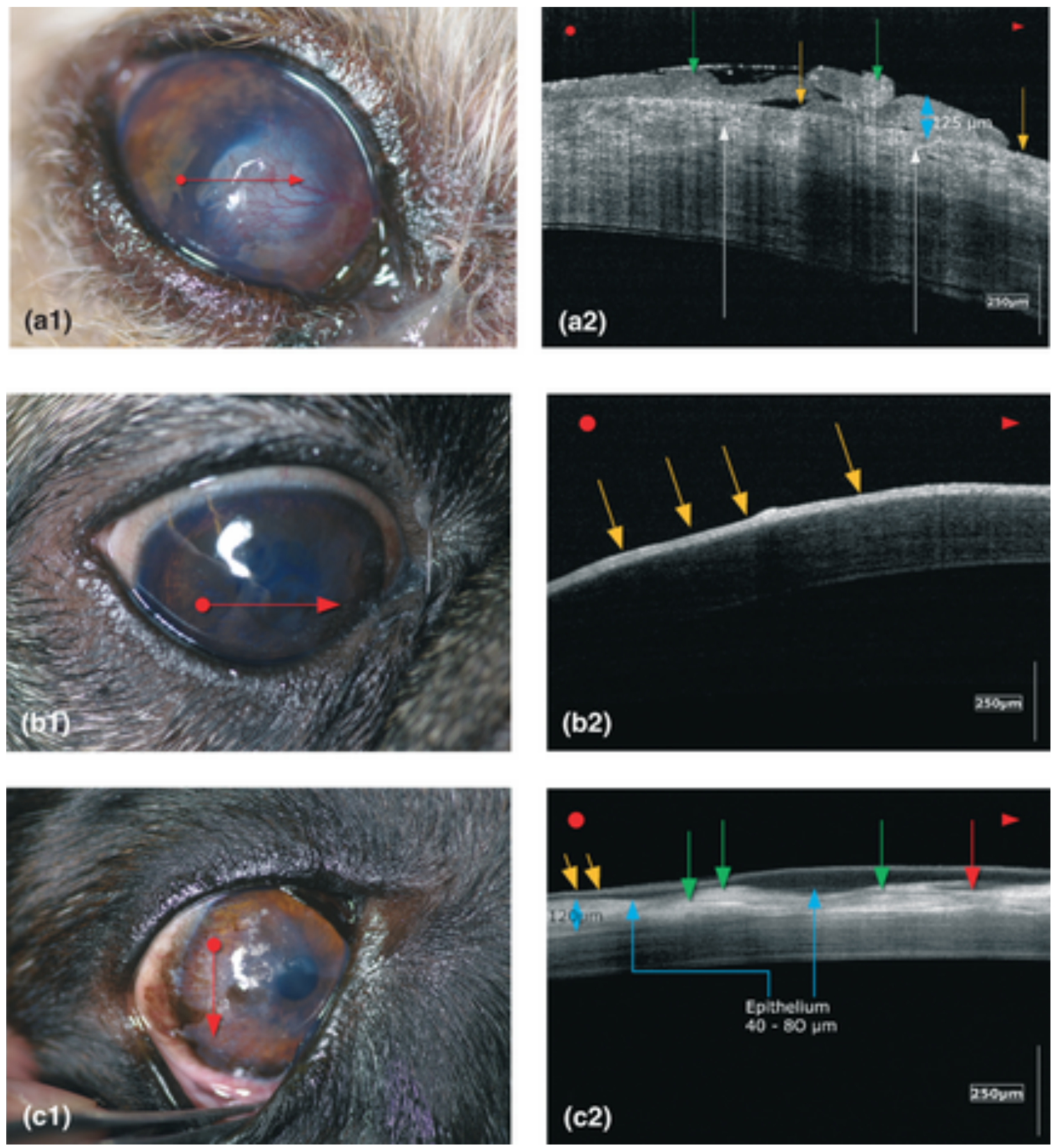

Figure 2.6: Macroscopic images showing B-Scan path (left) and corresponding cross-section (right) of the epithelial tissue in canine cornea. [16]

Depending on the type of source used for OCT, the system topology can change slightly. For Swept-Source OCT (SS-OCT), the topology is identical to the FMCW LIDAR topology shown in Figure 2.1. SS-OCT A-Scans employ a fixed reference path length and a swept source wavelength, and measure the spectral response of the interferometer encoded as an interferogram in optical frequency space. An interferogram is a sum of oscillations with different periods corresponding to reflections at different depths. 
By taking the Fourier transform of this interferogram, the reflectivity profile as a function of the depth of the sample is revealed. [33] Because SS-OCT uses fixed path lengths and an akinetic swept source such as a SG-DBR laser it is possible to perform A-Scans and B-Scans at high repetition rates. This leads to the possibility of real-time imaging of three-dimensional structures. A current SS-OCT system manufactured by Thorlabs offers A-Scan rates as high as $100 \mathrm{kHz}$, which allows for B-Scan rates higher than $1 \mathrm{kHz}$ and CScan rates higher than $1 \mathrm{~Hz}$. The aforementioned system offers penetration depths of $12 \mathrm{~mm}$ with a resolution of $16 \mu \mathrm{m}$. [36]

There are some notable differences between FMCW LIDAR and SS-OCT techniques. In SS-OCT, the distance to the sample is known, fixed, and very close to the source. Additionally, since high-resolution imaging is crucial to OCT, the source must sweep over a much larger optical bandwidth compared to LIDAR applications. On the contrary, target distance in LIDAR applications are unknown, potentially changing, and usually relatively far from the source. Since the distance to the target is so much larger, resolution can be much less precise. This allows for a smaller FMCW bandwidth requirement to achieve this resolution. The ratio of range resolution to range should be on order of $10^{-3}$ to $10^{-4}$ in a practical imaging system, so the range resolution can be increased for long-range imaging systems without degrading system performance. For example, most applications imaging an object at a range of $1 \mathrm{~km}$ do not require a range resolution at the micrometer or centimeter scale: a range resolution of 1 meter would probably suffice. 


\section{Chapter 3: VT-DBR Laser Background}

\subsection{VT-DBR functionality}

Vernier Tuned Distributed Bragg Reflector (VT-DBR) lasers were originally developed to improve optical communications through dense wavelength division multiplexing (DWDM). SG-DBR lasers are good candidates for WDM because they can operate at over ninety $50 \mathrm{GHz}$ spaced optical channels with over $40 \mathrm{~dB}$ side-mode suppression and output power over $10 \mathrm{~mW}$. [20] The laser was used for OCT and LIDAR applications only after it was found to be able to produce continuous linear wavelength sweeps over its wide tuning range. Producing these linear sweeps in VT-DBR lasers has proven challenging and a great deal of research has been done to improve their linearity while maintaining high repetition rates. $[4,9,31,32,34]$

The VT-DBR laser is a monolithic semiconductor device, typically with five distinct sections on the laser chip. Each of these sections is a separate PIN diode. Figure 3.1 shows a top-down and side view of the VT-DBR laser chip with the different sections labeled, while Figure 3.2 shows both a macroscopic and microscopic view of the laser chip.

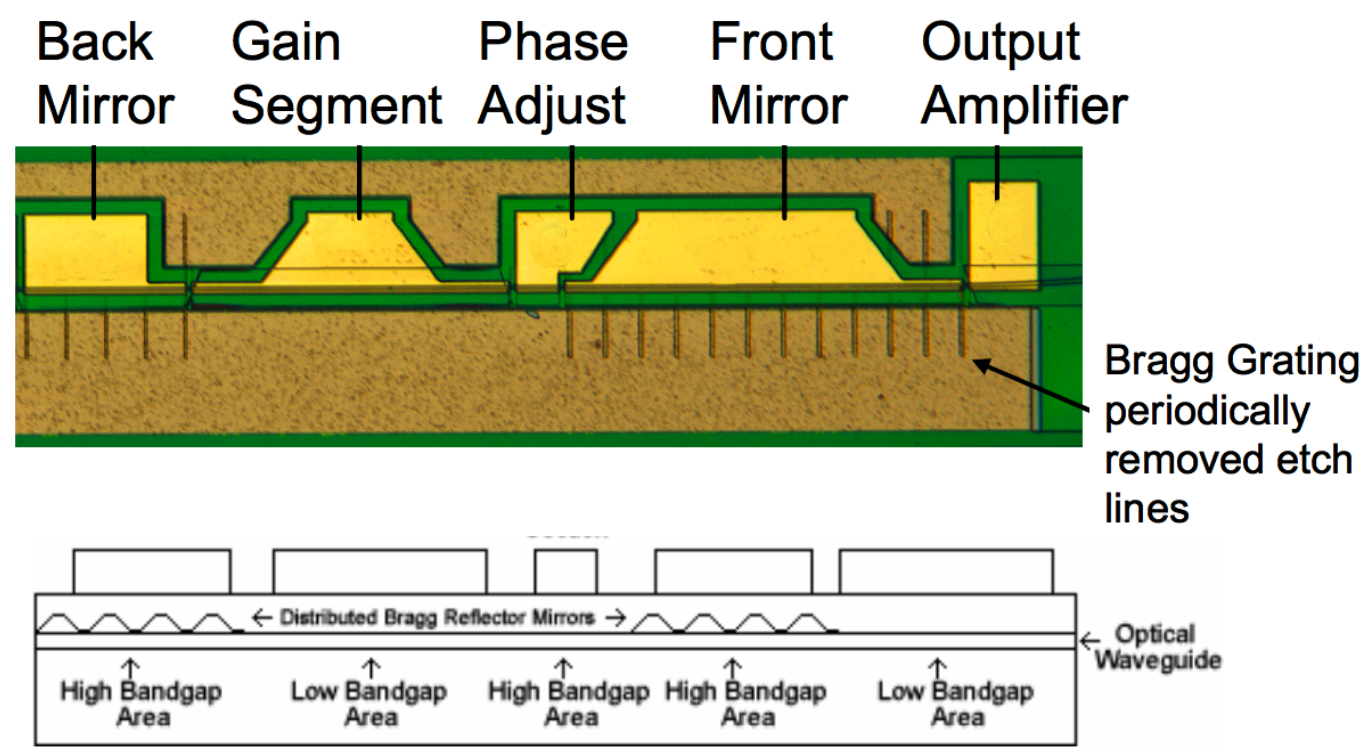

Figure 3.1: Top-Down (above) and side (below) view of the VT-DBR laser chip showing the various sections of the laser. [9] 

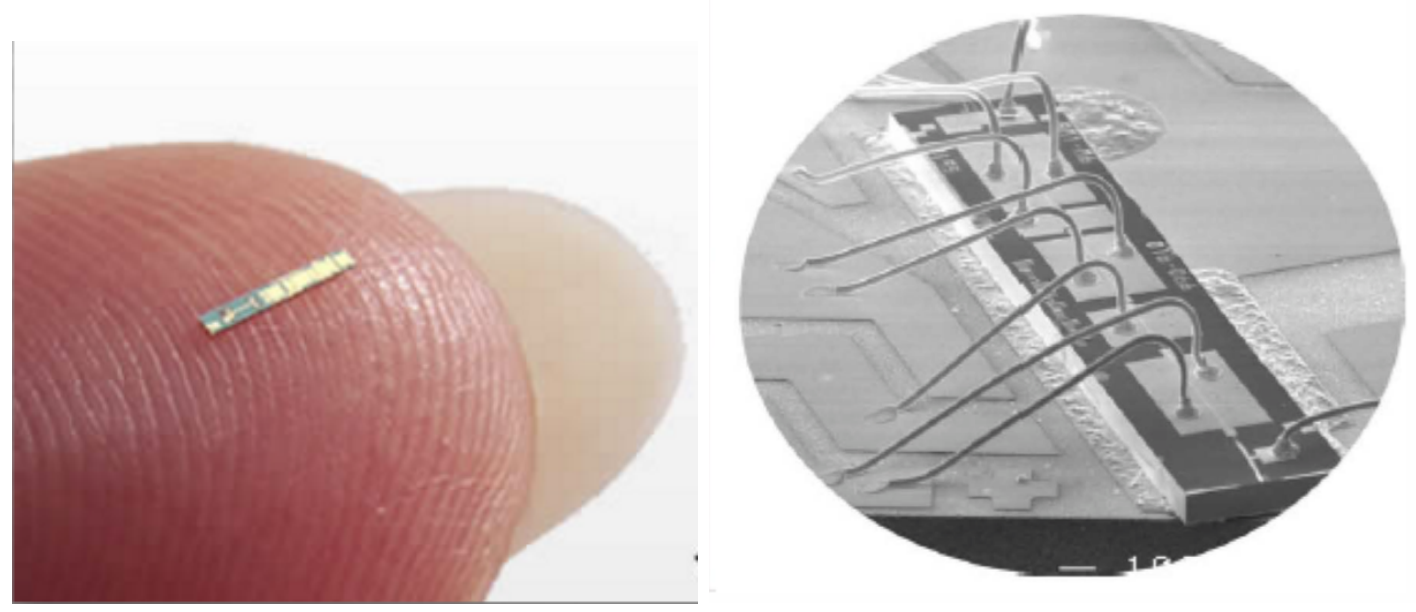

Figure 3.2: Macroscopic (left) and microscopic (right) view of a VT-DBR laser chip to show size. Rightmost image shows wire bonding for current injection into each section. [18]

The five sections of the laser can be categorized into two distinct groups based on function. The gain and semiconductor optical amplifier (SOA) sections are called active sections because they provide the mechanism for stimulated emission required for lasing. The laser itself only requires the gain section, but the SOA section is integrated for increased output power. The active sections provide optical gain via low band-gap material that is controlled by current injection into each section, similar to a basic semiconductor laser. Figure 3.3 shows how stimulated emission occurs as increased currents are injected. Past a threshold current, $\mathrm{I}_{\mathrm{th}}$, stimulated emission processes take over and lasing occurs.
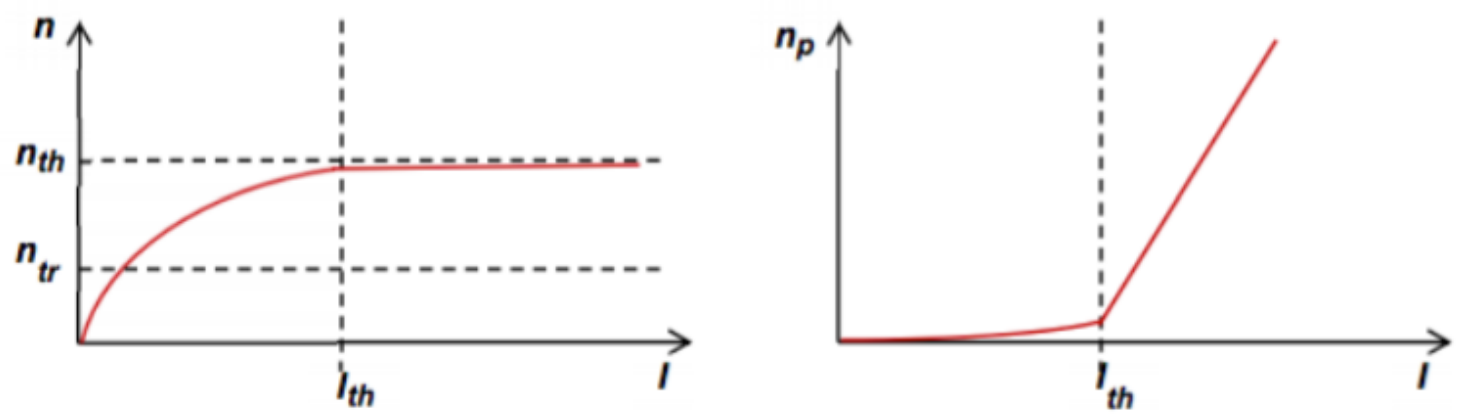

Figure 3.3: Carrier density as a function of injected current (left) and photon density as a function of injected current (right) for a semiconductor laser. [14]

The remaining three sections are the back mirror, front mirror, and phase sections; these are called passive sections because they do not provide any gain. These sections are made with high band-gap materials in order to reduce losses at the lasing wavelength. The purpose of the passive sections is to provide a 
wavelength tuning mechanism via current injection. The front and back "mirrors" are actually PIN diodes with etched sampled Bragg gratings with slightly different spacing, which provide a coarse wavelength tuning mechanism. Sampled Bragg gratings provide a comb-like spectrum of reflectivity vs. wavelength that is dependent on the lasing wavelength $\lambda$, the effective index of refraction $n$, and the spacing between etches in the grating $L$. The inter-peak spacing is described by Bragg's law and shown in Equation 3.1. [9]

$$
\Delta \lambda=\frac{\lambda^{2}}{2 n L}
$$

For an average index of refraction of 3.3 and $5 \mathrm{~nm}$ spacing between reflectivity peaks, the spacing $L$ between etches in the grating is found to be $72 \mu \mathrm{m}$. The reflectivity spectrum is shown in Figure 3.4 for two different but closely spaced sampled gratings.

Back Mirror
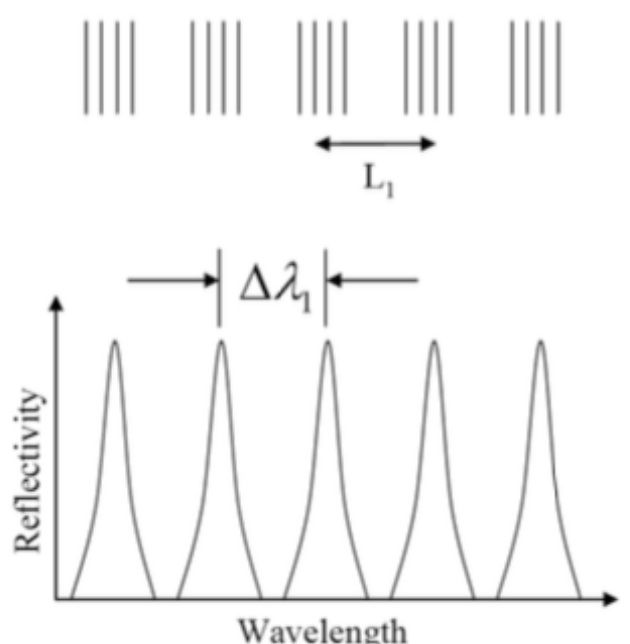

Front Mirror
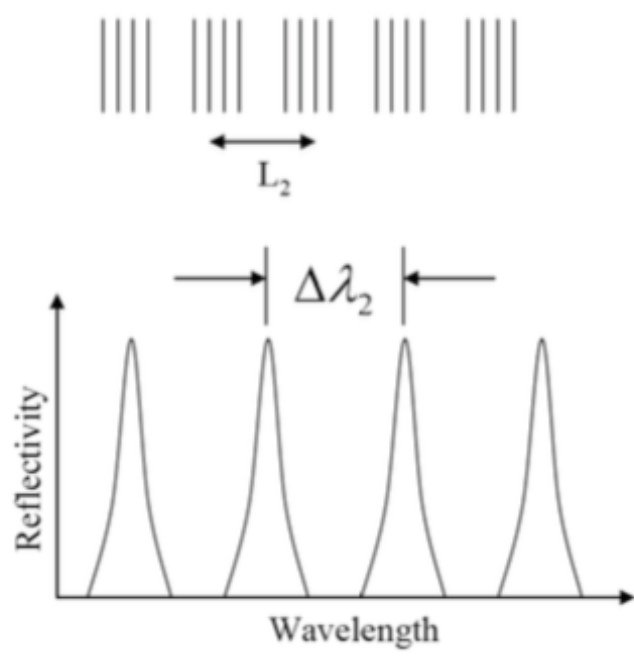

Figure 3.4: Reflectivity spectrum of two sampled Bragg reflectors with spacing $L_{1}>L_{2}$ and corresponding $\Delta \lambda_{1}<\Delta \lambda_{2}$. [4] 
Lasing will only occur at wavelengths where reflectivity peaks of both reflectors line up. Because the spacing of reflectivity peaks is only slightly different between the two mirror sections, only one set of peaks will ever be aligned. The wavelength at which the reflectivity peaks line up is the lasing wavelength. This tuning mechanism is known as the Vernier Effect, which is the namesake of the VT-DBR laser. This effect is shown in Figure 3.5. Since the spacing $L$ is a fixed value for each reflector, the effective index $n$ is used to tune each section. The effective index of refraction of a PIN diode is a function of carrier density, so it is possible to change $n$ with current injection. It is thus possible to tune the laser wavelength by current injection into one or both reflector sections.

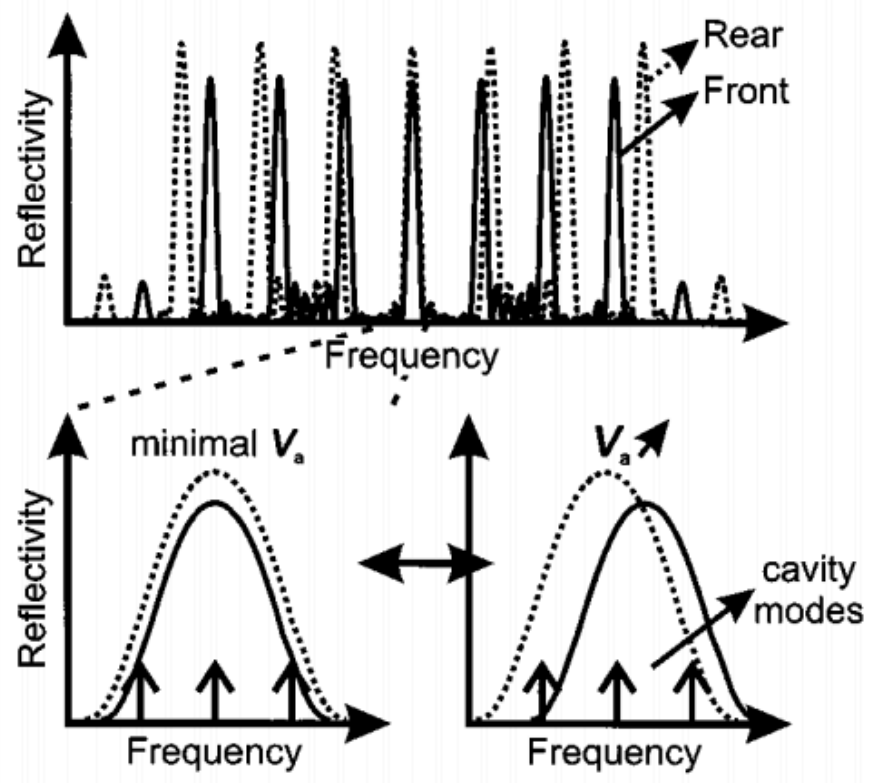

Figure 3.5: Vernier Effect for two Bragg reflectors with close spacing (Top). Both of the plots on the bottom lead to stable operation, but a slight misalignment of the reflectivity peaks (bottom right) will lead to decreased output power with more narrow linewidth. [17]

The phase section is similarly tuned with current injection, changing the effective index of refraction. The difference, however, is that the phase section effectively changes the cavity length of the laser. This mechanism is used to fine tune the laser wavelength around an operating point set by the current levels into the reflector sections. By injecting specific current waveforms into each passive section, it is possible to create a continuous wavelength sweep over the entire tuning range of the laser. Figure 3.6 shows these possible tuning paths to achieve full continuous tuning. 


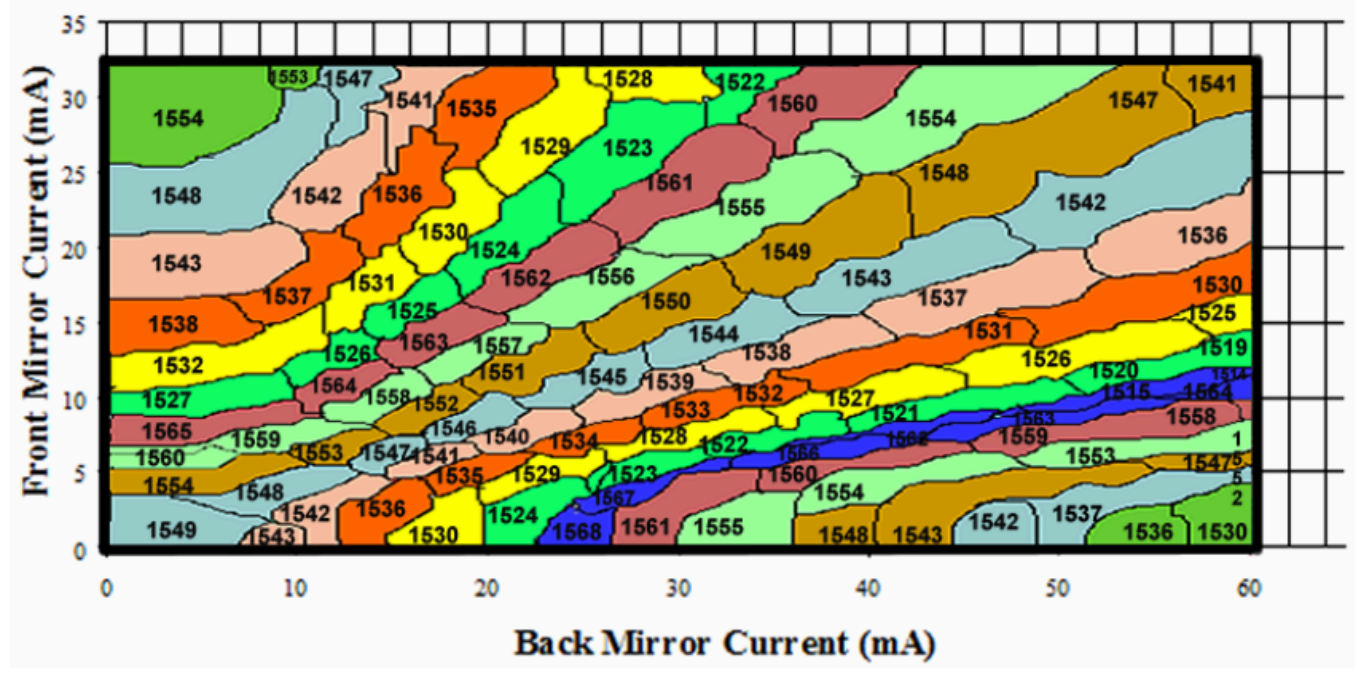

Figure 3.6: Example tuning map for a VT-DBR laser. The number enclosed in each region, colloquially called a "lily-pad", is the wavelength in nanometers. It is possible to consecutively tune to each wavelength in the tuning range by following these tuning paths, given by color. [9]

\subsection{Single Mirror Tuning}

Current injection into only one of the mirror sections leads to the widest tuning range, but this range is not continuous. The wavelength will change depending on which reflectivity peaks are lined up: with one set of peaks shifting and the other stationary the wavelength will change slightly as peaks become misaligned, until another set of peaks is better aligned. When this happens, a "mode hop" occurs and the lasing wavelength will shift by $\Delta \lambda$, approximately $5 \mathrm{~nm}$ in the example given. Figure 3.7 shows an example of single mirror tuning by increasing the injected current on the front mirror only. Note that a similar wavelength shift would occur for an equal decrease of injected current into the back mirror. 

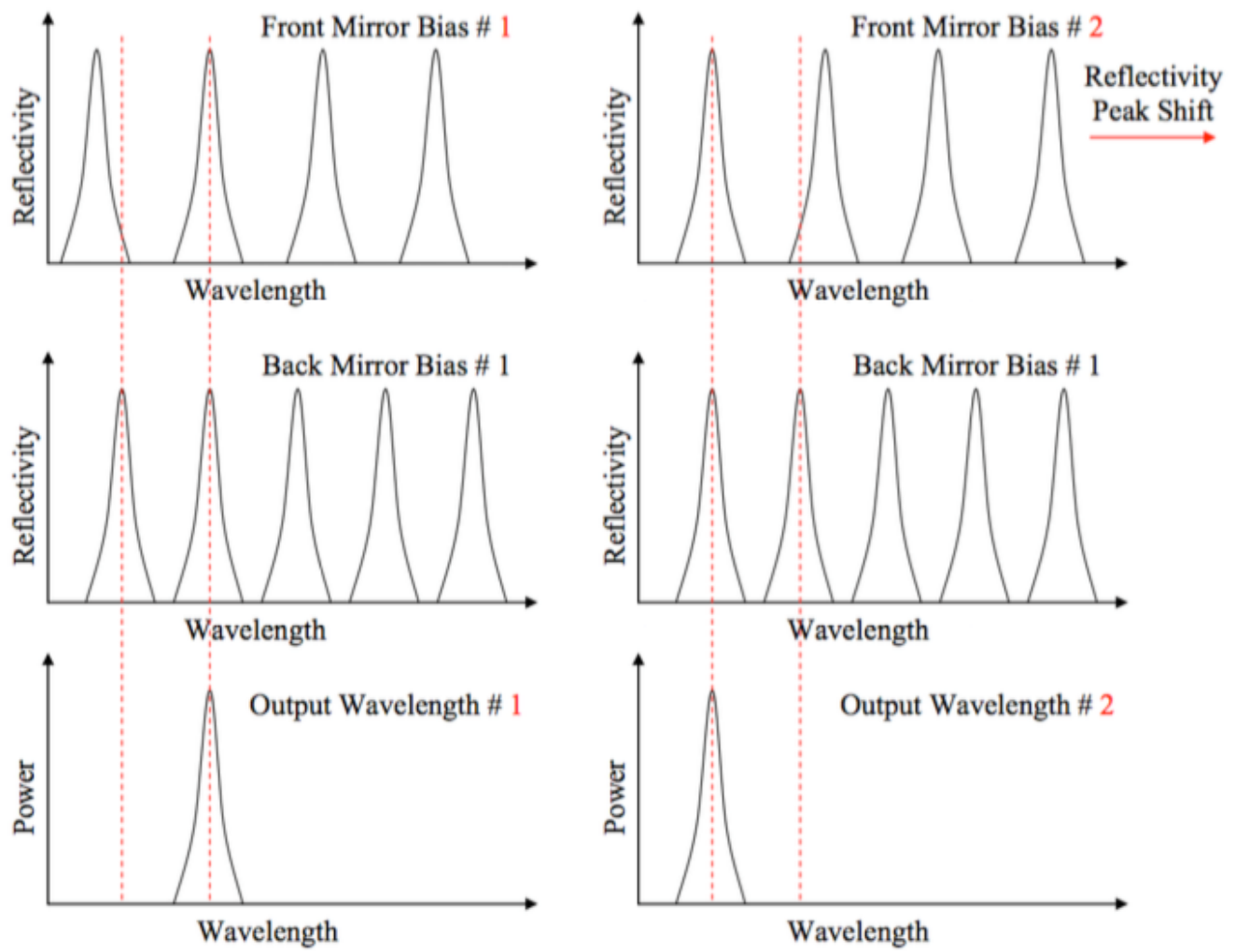

Figure 3.7: Single mirror tuning via an increased front mirror current and a constant back mirror current. This tuning method leads to large and discontinuous jumps in wavelength, called "mode hops" given by the inter-peak spacing in the reflectivity spectrum of the mirror being tuned. [4]

\subsection{Simultaneous Tuning of Front and Back Mirrors}

If instead both mirror sections are biased simultaneously, it is possible to achieve a much finer adjustment of the lasing wavelength. Instead of the $5 \mathrm{~nm}$ mode hops caused by the alignment of a new pair of reflectivity peaks, much finer mode hop of $300 \mathrm{pm}$ is seen to occur. This $300 \mathrm{pm}$ mode hop is due to the cavity mode spacing of the VT-DBR laser. Simultaneous tuning of the mirror sections is shown in Figure 3.8. Note that while these mode hops are much smaller, they still occur in discrete steps. Therefore this method is not sufficient for continuous tuning. 


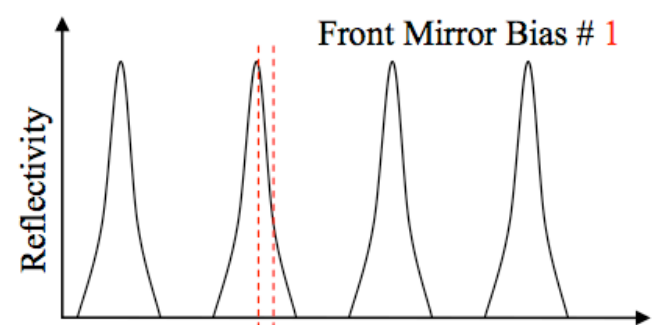

Wavelength

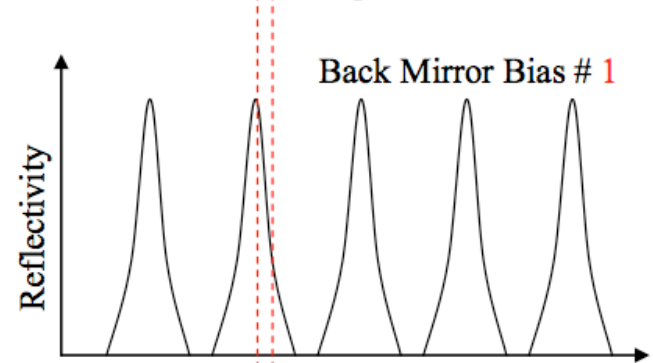

Wavelength

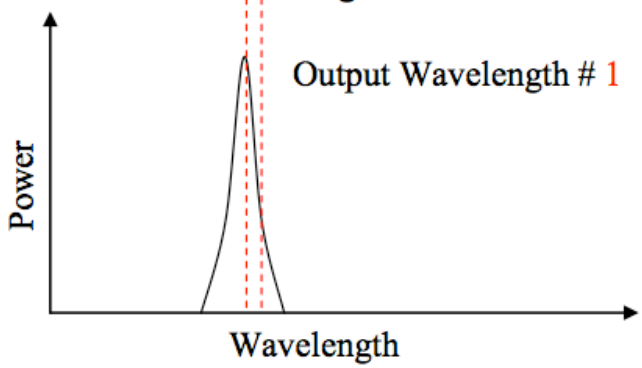

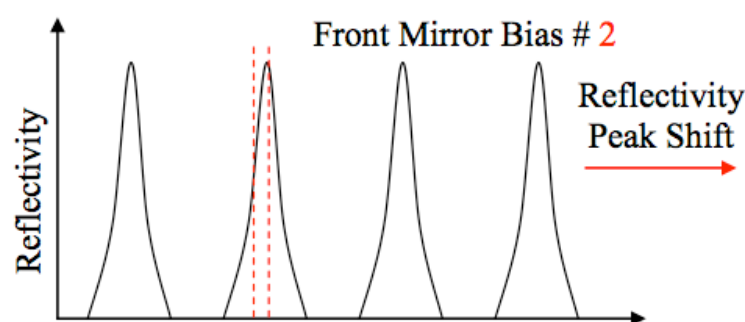

Wavelength

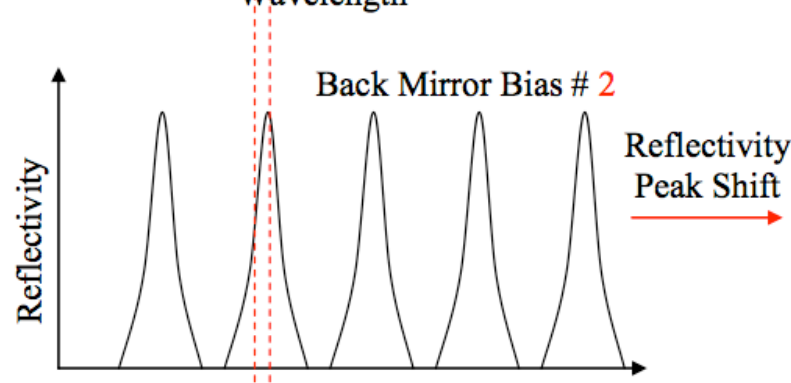

Wavelength

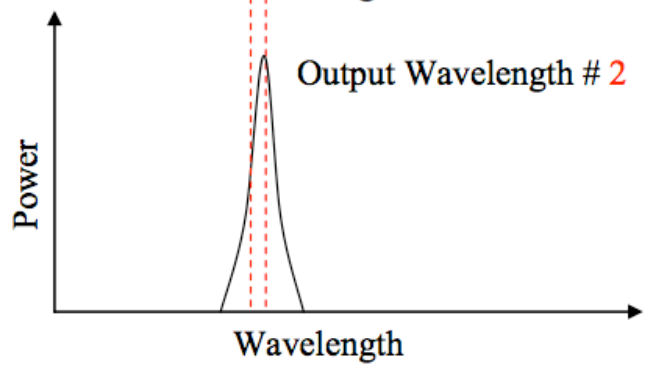

Figure 3.8: Simultaneous mirror tuning via a simultaneous increase of front and back mirror current. This tuning method leads to smaller but still discontinuous jumps in wavelength of approximately 300 picometers caused by the cavity mode spacing of the laser. [4]

\subsection{Simultaneous Tuning of Front Mirror, Back Mirror, and Phase sections}

To achieve truly continuous wavelength tuning, all three passive sections must be used. The addition of the phase section currents allow for continuous adjustment of the cavity mode spacing, which allows for continuous tuning through the wavelengths that could not be achieved through biasing only the mirror sections. A decrease in phase section current leads to an increase in the lasing wavelength, but only for a small bandwidth. By using the $300 \mathrm{pm}$ cavity mode hops in conjunction with the cavity length adjustment given by the phase section, it is possible to achieve a continuous and nearly linear wavelength tuning ramp.

\subsection{Linewidth Considerations}

While it is possible to tune the laser continuously through any wavelength in its tuning range, the laser linewidth is by no means a constant over that range. One heuristic is that increased currents into the passive sections will lead to increased laser linewidth. This is due to increased shot noise, which becomes 
noise on the section's index of refraction. This is true, but paints an incomplete picture. [23] Figure 3.9 shows the simulated and measured laser linewidth as a function of both front and back mirror current. The simulation shows a general trend of linewidth broadening with increased currents, but the experimental data shows less general linewidth broadening with various spikes of very wide linewidth.
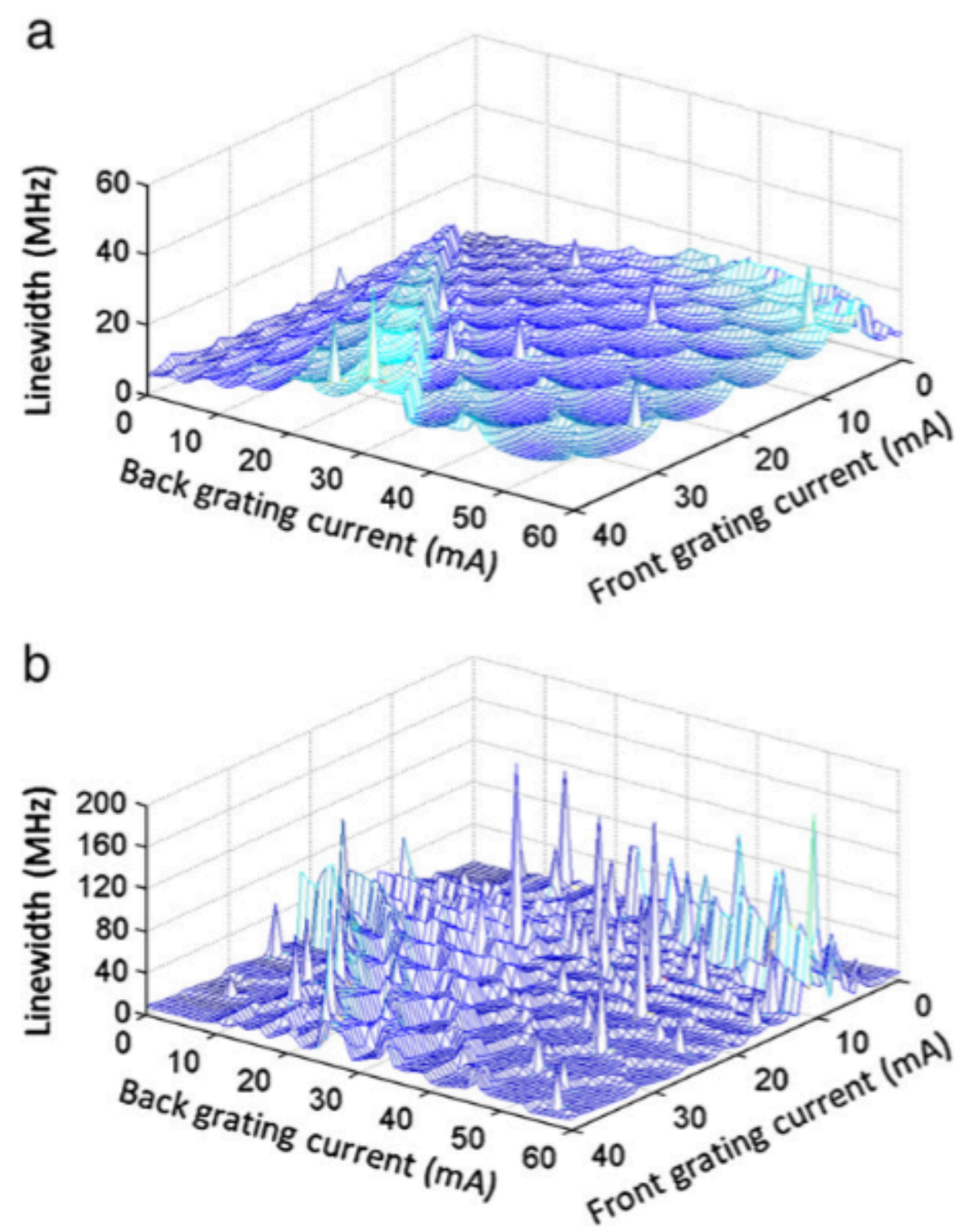

Figure 3.9: Simulated (a) and measured (b) linewidth for various front and back mirror currents. Note that $I_{F M}=I_{B M}=0 \mathrm{~mA}$ is in the upper corner of each plot. Simulation shows a trend of linewidth broadening with increased current, while experimental data shows less general linewidth broadening with narrow regions of much larger linewidth. [20] 
Given the experimental data in Figure 3.9 (b), it would be nearly impossible to construct a tuning path over the entire wavelength range of the laser with a constant and narrow linewidth. One can imagine, however, that there are smaller regions of tuning within this map that do have a constant and narrow linewidth. One purpose of this work is to find and characterize these narrow-linewidth tuning paths in each of three modes of VT-DBR operation. Figure 3.10 shows three possible regions on a VT-DBR tuning map, labeled A, B, and $\mathrm{C}$.

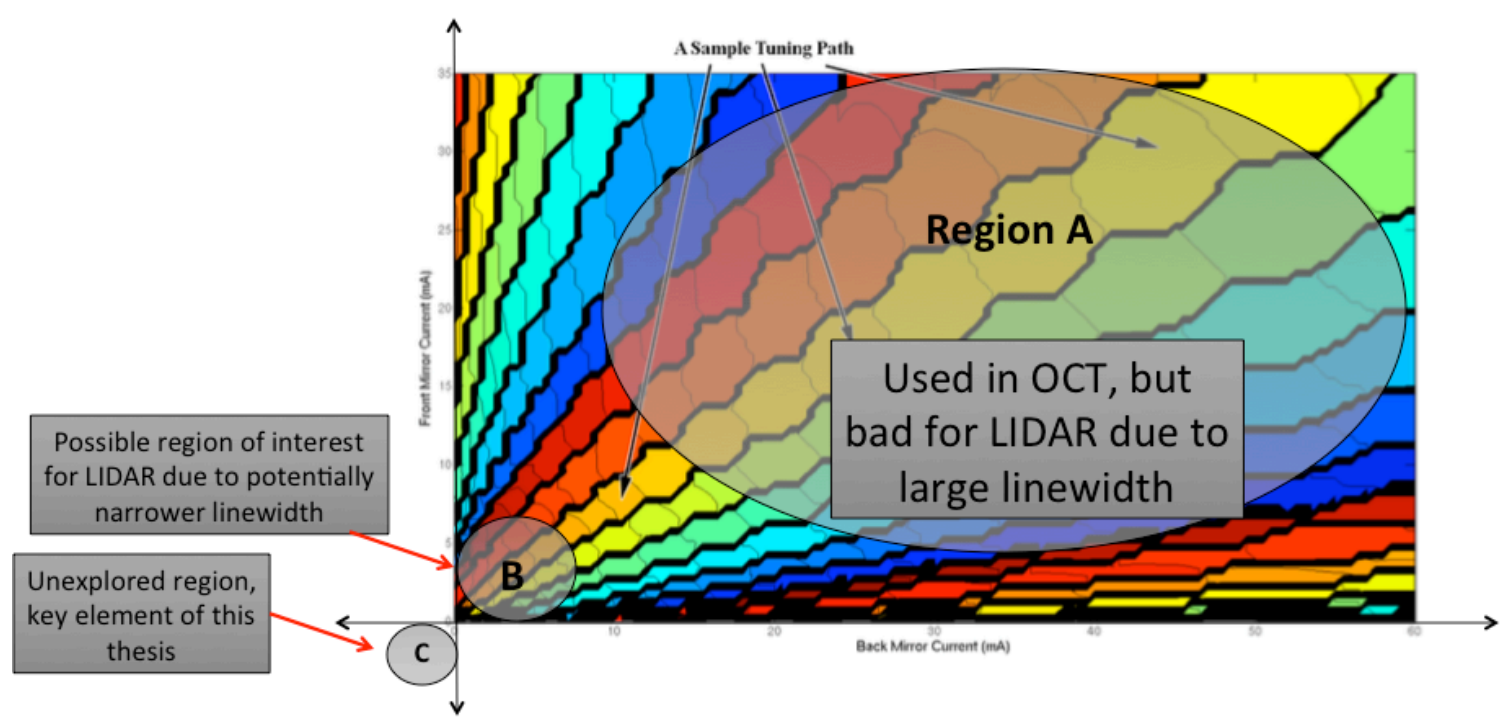

Figure 3.10: Three possible VT-DBR modes of operation

Region A is used in OCT imaging because it provides a maximal continuous tuning range, but it is bad for LIDAR due to its large linewidth. Region B is a potentially interesting region because it provides a wide tuning range with potentially narrower linewidth due to decreased shot noise. Finally, region $\mathrm{C}$ is an unexplored area of reverse biasing and is a key element of this work. 


\section{Chapter 4: Common Sources of Noise in Optical Measurements}

The following analysis of common noise sources can be found in [8]

\subsection{Electrical Thermal Noise}

One common source of noise that must be considered in almost any detection process is the thermal noise generated in the receiver electronics. The resistance first experienced by the photocurrent generated by the detector generates this thermal noise, also known as Johnson noise. A simplified model of a photodiode connected to an electrical amplifier and its equivalent circuit with thermal noise included is shown in Figure 4.1.

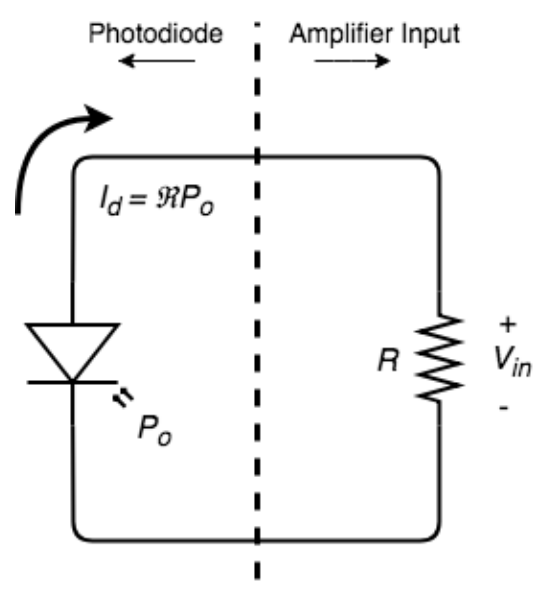

(a)

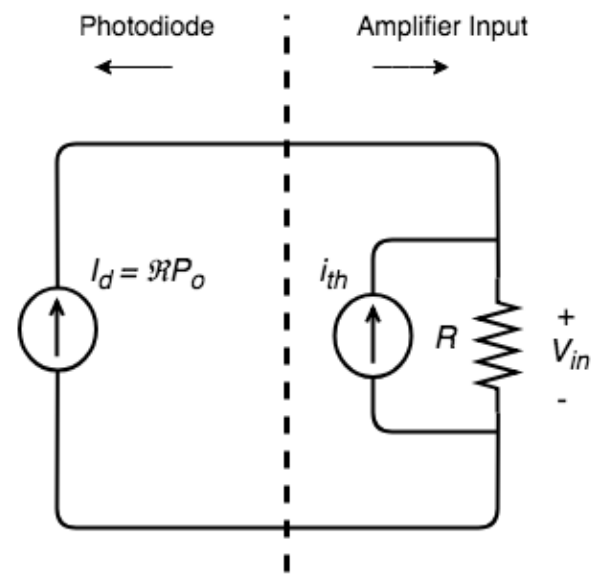

(b)

Figure 4.1: (a) Simplified diagram of a photodiode connected to an electrical amplifier. (b) The equivalent circuit model including thermal resistive noise using ideal current sources.

In this model, $P_{o}$ is the optical power incident on the photodiode, $\Re$ is the responsivity of the photodiode with units of [A/W], $I_{d}$ is the photodiode current, $R$ is the input resistance of the amplifier, and $V_{\text {in }}$ is the input voltage signal to the amplifier. There is a thermally generated rms current noise $\hat{\imath}_{t h}$ in a $1 \mathrm{~Hz}$ bandwidth, given by Equation 4.1 .

$$
\hat{\imath}_{t h}=\sqrt{\frac{4 k T}{R}} \quad[A / \sqrt{H z}]
$$

In Equation 4.1, $k=1.38 \times 10^{-23} \mathrm{~J} / \mathrm{K}$ is Boltzmann's constant and $T$ is the temperature of the resistor in Kelvin. The caret $(\wedge)$ above the rms current symbol is to indicate that the current noise is normalized to a 
$1 \mathrm{~Hz}$ bandwidth. The total rms current noise is found by multiplying Eq. 4.1 by the square root of the receiver bandwidth, $i_{t h}=\hat{\imath}_{t h} \sqrt{\Delta f}$.

\subsection{Optical Intensity Noise}

Another form of noise often encountered in optical measurements is the intensity noise on the optical signal even before the detection process. This intensity noise is fundamentally caused by optical interference between the stimulated laser signal and spontaneous emission generated within the laser cavity, but laser sources like distributed feedback (DFB) lasers, Fabry-Perot (FP) lasers, and VT-DBR lasers typically exhibit intensity noise levels that are dependent on pump levels and feedback conditions. External feedback, such as back reflections through the fiber into the laser cavity, can cause large variations in intensity noise.

A useful and convenient way to describe and compare intensity noise is to express it as a ratio of noise power in a $1 \mathrm{~Hz}$ bandwidth normalized by the DC signal power. This quantity is independent of attenuation or the absolute power reaching the photo-detector. This fractional noise power per bandwidth is often called relative intensity noise (RIN) and is defined as

$$
R I N=\frac{<\Delta \hat{\imath}^{2}>}{I_{d c}^{2}} \quad\left[\mathrm{~Hz}^{-1}\right]
$$

where $\left\langle\Delta \hat{\imath}^{2}\right\rangle$ is the time-averaged intensity noise power in a $1 \mathrm{~Hz}$ bandwidth and $I_{d c}$ is the average DC intensity. Since RIN is a normalized parameter, Equation 4.2 is equally valid if $\Delta \hat{\imath}$ and $I_{d c}$ refer to optical intensity, detected photocurrent, or receiver output voltage. In practice, RIN is most easily calculated using an electrical spectrum analyzer to measure $\left\langle\Delta \hat{\imath}^{2}\right\rangle$ and a DC ammeter to measure $I_{d c}$. RIN is generally a function of frequency, but for a flat noise spectrum the total rms current noise caused by RIN is given by

$$
i_{R I N}=I_{d c} \sqrt{R I N \Delta f} \quad[A / \sqrt{H z}]
$$

where $\Delta f$ is the effective noise bandwidth of the receiver.

\subsection{Photocurrent Shot Noise}

Electrical shot noise is caused by the random arrival time of electrons that make up an electrical current. It becomes an important noise source when trying to measure a small signal in the presence of a large DC background, which is usually the case in coherent detection schemes such as FMCW LIDAR. The rms shot noise current in a $1 \mathrm{~Hz}$ bandwidth is given by 


$$
\hat{\imath}_{s n}=\sqrt{2 q I_{d c}} \quad[A / \sqrt{H z}]
$$

where $q=1.6 \times 10^{-19} \mathrm{C}$ is the charge of an electron and $I_{d c}$ is the DC photocurrent. The total rms shot current $i_{s n}$ is found by multiplying Equation 4.4 by the square root of the effective noise bandwidth of the receiver, $i_{s n}=\hat{\imath}_{s n} \sqrt{\Delta f}$.

Although RIN is defined as the fractional intensity noise on an optical signal, it can be used in an unconventional manner to describe the level of shot noise on a DC photocurrent. By dividing the shot noise current by the DC current and squaring this ratio, the result is an expression equivalent to RIN. This allows for easy comparison with other noise sources in the system. Combining Equations 4.2 and 4.4, shot noise produces an effective RIN given by

$$
\operatorname{RIN}_{s n}=\frac{2 q}{I_{d c}} \quad\left[\mathrm{~Hz}^{-1}\right]
$$

This result is useful for determining the required DC photocurrent to make an accurate measurement on an optical signal. To make an accurate RIN measurement, the detected DC photocurrent must be large enough to prevent shot noise from being the dominant noise source.

\subsection{Optical Phase Noise}

A final source of noise in optical systems is optical phase noise, or fluctuations in the frequency of the optical carrier. For systems that use an interferometer to mix a time-delayed signal with a reference signal, these frequency modulations are directly converted into intensity modulation. The magnitude of this intensity modulation is dependent on the slope of the frequency discriminator at the frequency of interest. This conversion is shown in Figure 4.2 for a quadrature point where the slope is a maximum. No conversion happens at points of zero slope. The detected photocurrent for a cw laser signal passed through an interferometer can be written as

$$
I_{d}(v ; t)=\mathfrak{R} P_{o}\left[1+R_{p}+2 \sqrt{R_{p}} \cos \left(2 \pi \tau_{o} v(t)\right)\right][A],
$$

where $\Re$ is the responsivity of the photo-detector, $P_{o}$ is the optical power into the interferometer, $\tau_{o}$ is the time delay of the interferometer, $R_{p}$ is the ratio of the two interfering optical powers, and $v(t)$ is the instantaneous optical frequency of the laser source. 


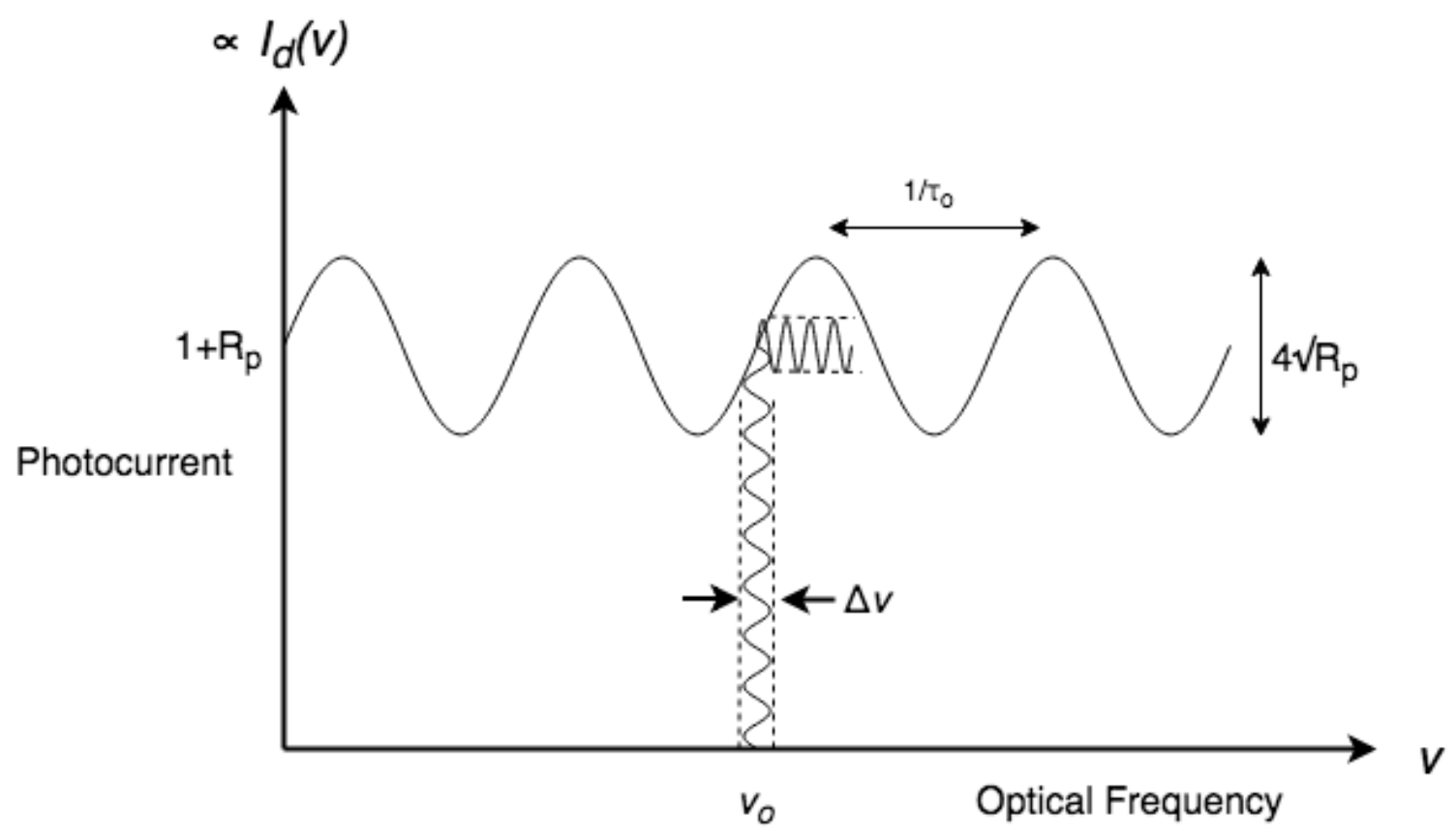

Figure 4.2: Conversion of frequency modulation to intensity modulation for an optical signal passed through an interferometer with time delay $\tau_{o}$. Amplitude of intensity modulation depends on slope of frequency discriminator at the frequency of interest. No conversion happens at points of zero slope.

The maximum slope occurs at the quadrature position shown in Figure 4.2, and the fractional intensity change caused by a small frequency change $\Delta v(t)$ at quadrature is given by

$$
\frac{\Delta I(t)}{I_{\text {avg }}} \cong K_{f m} \Delta v(t)
$$

where $K_{f m}=4 \pi \tau_{o} \sqrt{R_{p}} /\left(1+R_{p}\right)$ is the maximum slope from Equation 4.4 and can be considered the FM discriminator constant. Since Equation 4.4 is a linear approximation derived from a non-linear expression, restrictions on both the magnitude and modulation frequency on $\Delta v(t)$ are required for the approximation to be accurate.

The FM discriminator process described above can also convert a laser linewidth into a spectral noise density on the detected photocurrent. This effect is quite different for coherent interference, where the coherence length of the laser is much longer than the path length difference experienced by the interfering signals, and incoherent interference, where the coherence length of the laser is much less than the aforementioned path length difference. For each case, a distinct equation can be derived for maximum RIN 
due to the conversion of optical phase noise into intensity noise. Equations 4.6 and 4.7 give the RIN expressions for coherent and incoherent interference.

$$
\begin{gathered}
R I N_{\Delta \phi, \text { coherent }} \cong \frac{2 R_{p}}{\left(1+R_{p}^{2}\right)} 8 \pi \tau_{o}^{2} \Delta v_{l w} \operatorname{sinc}^{2}\left(\tau_{o} f\right) \quad\left[\mathrm{Hz}^{-1}\right], \\
R I N_{\Delta \phi, \text { incoherent }}=\frac{2 R_{p}}{\left(1+R_{p}^{2}\right)} \frac{2}{\pi \Delta v_{l w}} \frac{1}{1+\left(f / \Delta v_{l w}\right)^{2}}\left[\mathrm{~Hz}^{-1}\right],
\end{gathered}
$$

Both the coherent and incoherent cases assume a Lorentzian laser lineshape with linewidth $\Delta v_{l w}$, and in Equation 4.6 the function $\operatorname{sinc}(x)=\frac{\sin (\pi x)}{\pi x}$ is used. It is important to note that Equation 4.6 is an approximation that constrains the laser linewidth to $\Delta v_{l w}<\frac{0.1}{\tau_{o}}$ to be reasonably valid. Additionally, the coherent case is sensitive to environmental variations in the bias conditions and could fluctuate between the maximum value given by Equation 4.6 and zero. The incoherent case is not sensitive to environmental variations and has a $-3 \mathrm{~dB}$ bandwidth given by the linewidth of the laser. The resulting power spectral density for each case is shown in Figure 4.3.

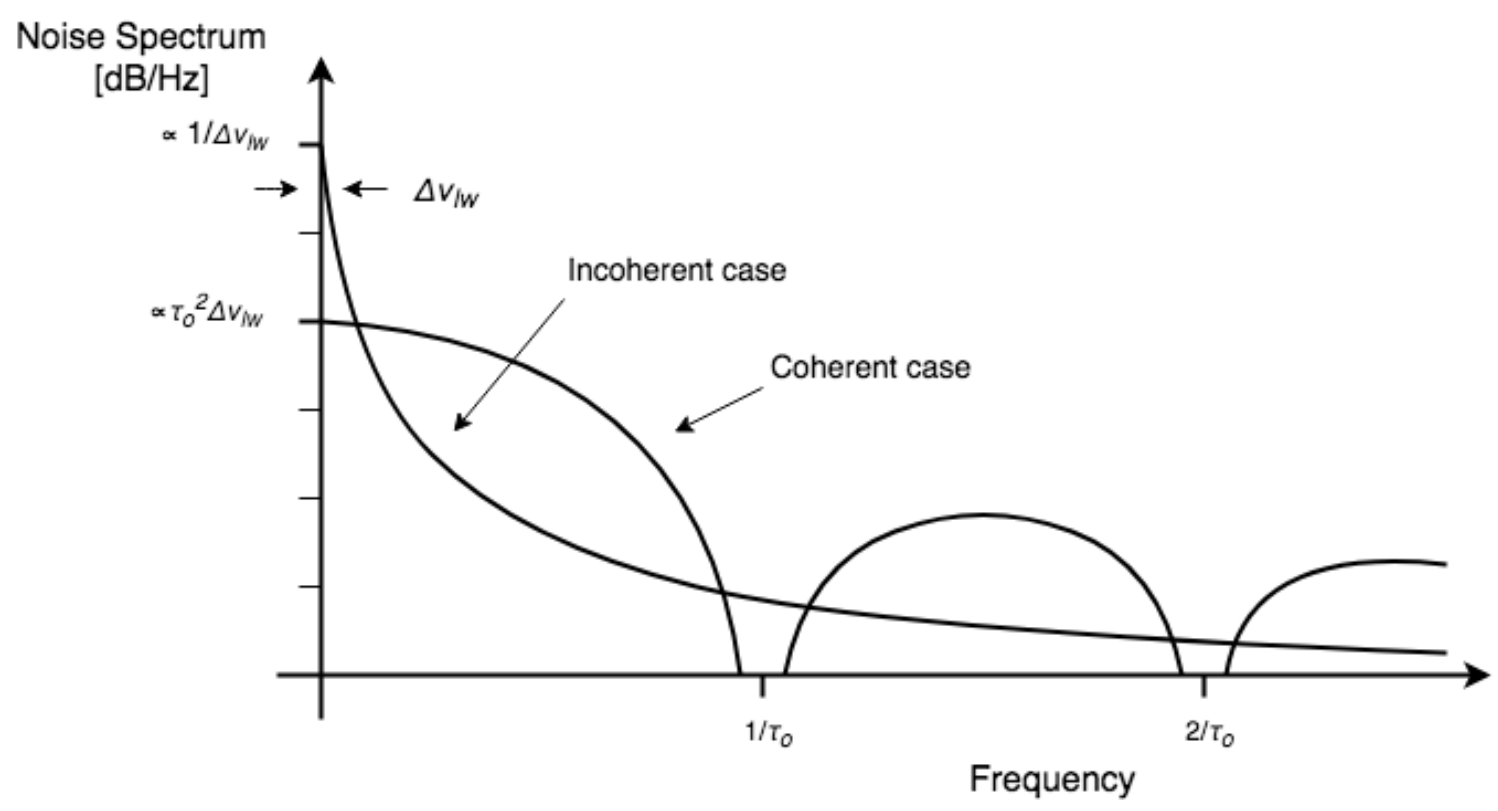

Figure 4.3: Power spectral density resulting from the conversion of laser phase-noise into optical intensity noise for both the coherent and incoherent interference. 


\subsection{Summary}

In a real optical measurement system, all four of the previously mentioned sources of noise would be present. To determine the total noise level of the system, the individual noise sources have to be combined. Since each source of noise is uncorrelated with respect to the others, summing the square of each term and taking the square root gives the total rms photocurrent noise. This is shown in Equation 4.8, where the four terms correspond to thermal noise, shot noise, intensity noise, and phase noise.

$$
i_{\text {total }}=\sqrt{\frac{4 k T \Delta f}{R}+2 q I_{d c} \Delta f+I_{d c}^{2} R I N \Delta f+I_{d c}^{2} R I N_{\Delta \phi} \Delta f} \quad[\mathrm{~A}] \quad \text { (Eq. 4.8) }
$$

The total rms photocurrent noise given by Equation 4.8 can be used to determine the sensitivity of the receiver to optical power. The minimum change in optical power that can be detected is calculated using

$$
\Delta P_{\min }=\frac{i_{\text {total }}}{\Re} \quad[\mathrm{W}]
$$

where $\Re$ is the responsivity of the photo-detector in units of $\mathrm{A} / \mathrm{W}$. This minimum power sensitivity is proportional to the square root of the detection bandwidth, $\sqrt{\Delta f}$.

For FMCW LIDAR systems, which use swept source interferometry to detect range and velocity of a target, all of these sources of noise are present but phase noise can often be the dominant source of noise seen on the detector. 


\section{Chapter 5: Experimental Apparatus}

\subsection{Finisar S7500 Laser}

The laser source used in this system was the S7500 C-Band (1530-1565 nm) Modulated Grating Y-Branch (MG-Y) type laser manufactured by Finisar. MG-Y lasers are a type of VT-DBR laser with a Yshaped topology. The S7500 uses a monolithic InP chip with an integrated semiconductor optical amplifier (SOA). This chip is packaged in a small, hermetically sealed package with an internal 40dB optical isolator and internal wavelength locker. The laser output is available through a polarization-maintaining single mode fiber pigtail shortened to about $15 \mathrm{~cm}$. The maximum output power is greater than $13 \mathrm{dBm}$ and the intrinsic Lorentzian linewidth is less than 5MHz. The lowest emission wavelength is $1527.6 \mathrm{~nm}$ and the highest emission wavelength is $1568.4 \mathrm{~nm}$. This laser was originally developed for DWDM fiber optic communication systems, but is also useful for test and measurement. [15]

\subsection{Breakout Board for Laser Biasing}

To easily control temperature and inject current into the different laser sections, a PCB breakout board was designed to provide easy access to each section through an SMA connection. This board made testing the laser much simpler and was invaluable to the measurement process. Figure 5.1 shows a breakout board with SMA connections for each laser section.

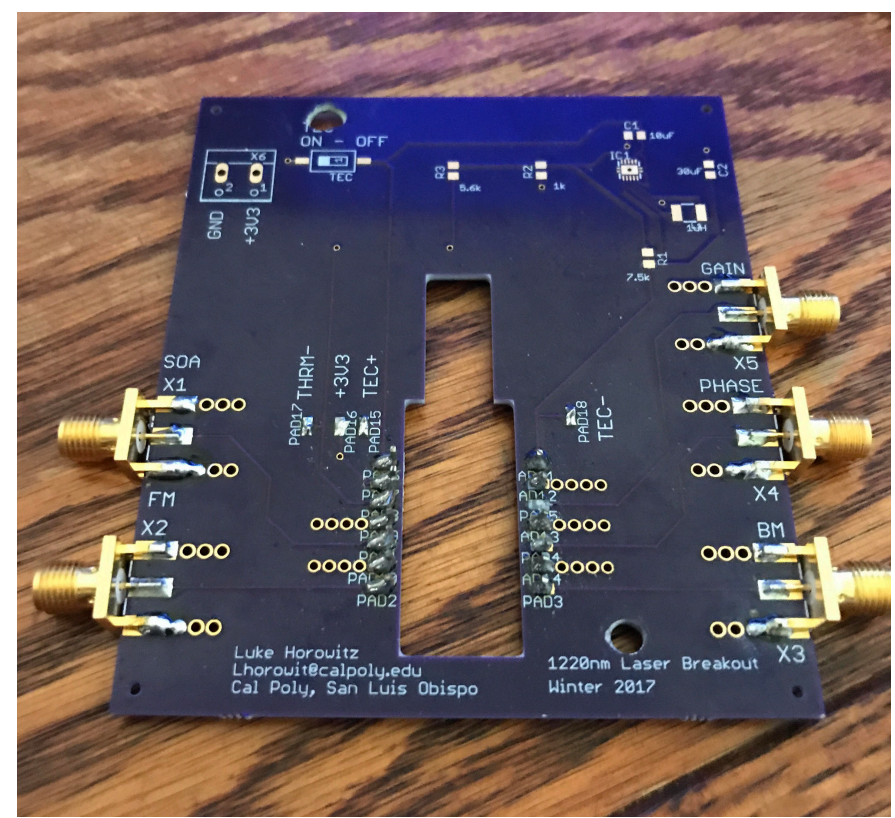

Figure 5.1: Breakout board with SMA edge-launch connections 
The breakout board was originally designed for a laser with a different pin-out, so an additional board is used to connect the Finisar S7500 to the breakout board. This second board also contains feedback circuitry for the optoelectronic feedback discussed in Chapter 7 and was designed by Insight Photonic Solutions.

Figure 5.2 shows the S7500 laser being temperature controlled and biased through the breakout boards, as well as the aluminum heat sink to which the laser is mounted.

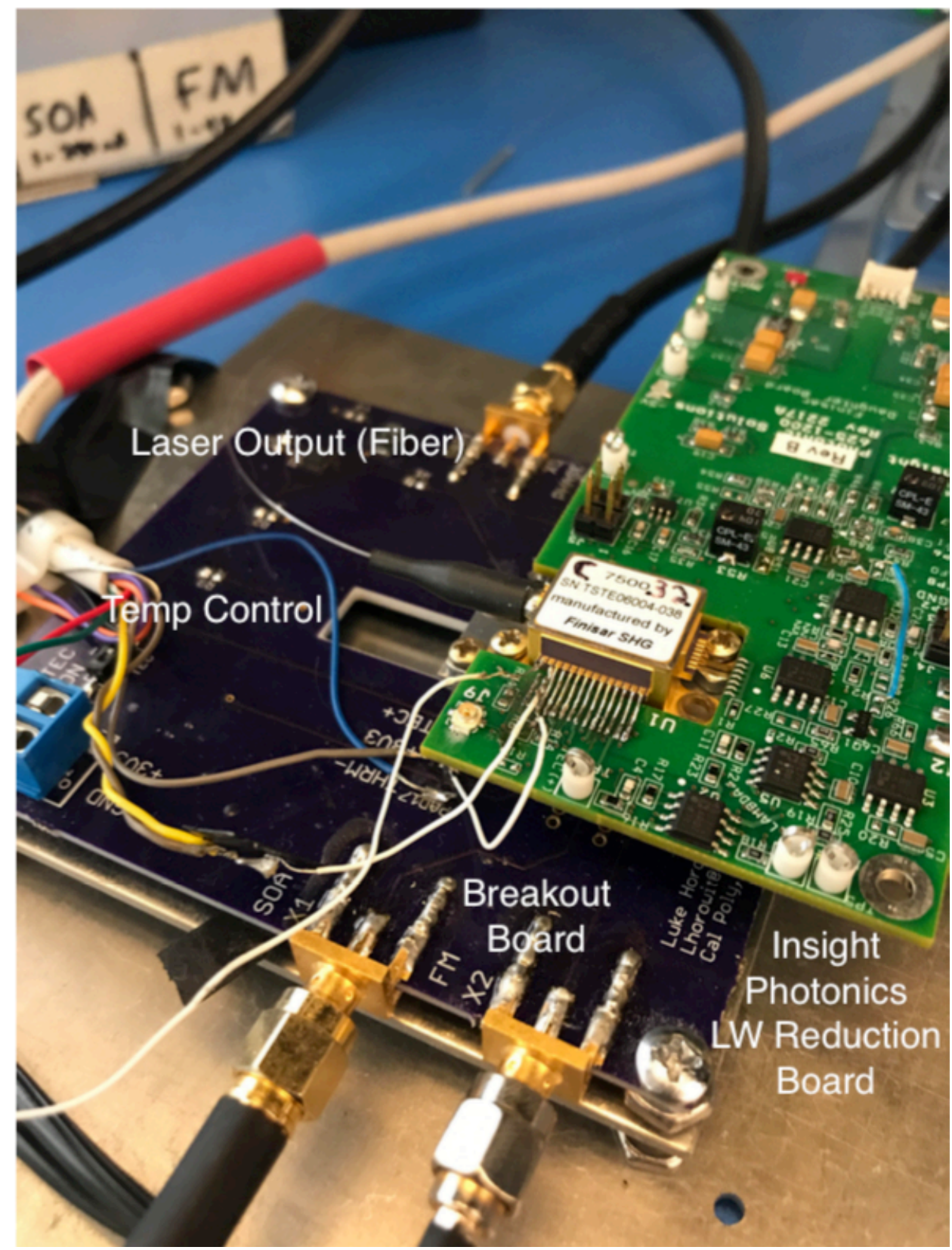

Figure 5.2: Laser and breakout boards on aluminum heat sink 


\subsection{Battery-Powered Current Supplies for Low Noise Biasing}

VT-DBR laser linewidth is very sensitive to noise on the current injected into both the active and passive sections. For this reason, a simple battery powered resistive current supply was created to bias each section within its current limit. Batteries provide a low-noise source of current that is ideal for laser biasing. The circuit diagram for this current supply is shown in Figure 5.3 and a picture of the built supply in the lab is shown in Figure 5.4. The resistor in each current path limits the maximum current output and provides a constant reference for current by monitoring the voltage across it. The potentiometer in each path provides a method of tuning, and the maximum potentiometer resistance will set the lower limit on current output. Both the voltage monitors and current outputs are available via coaxial connection.

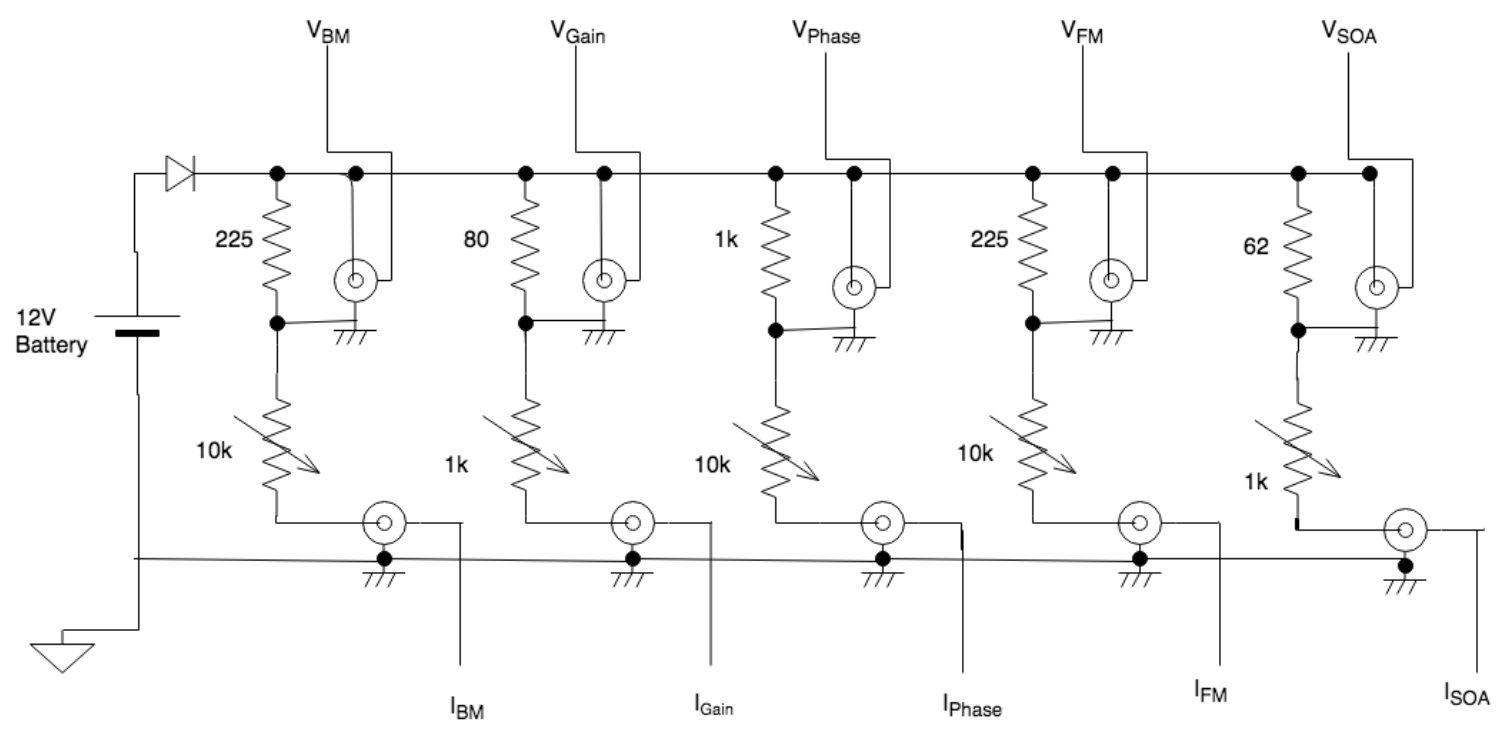

Figure 5.3: Circuit diagram for full-range battery powered current supply 


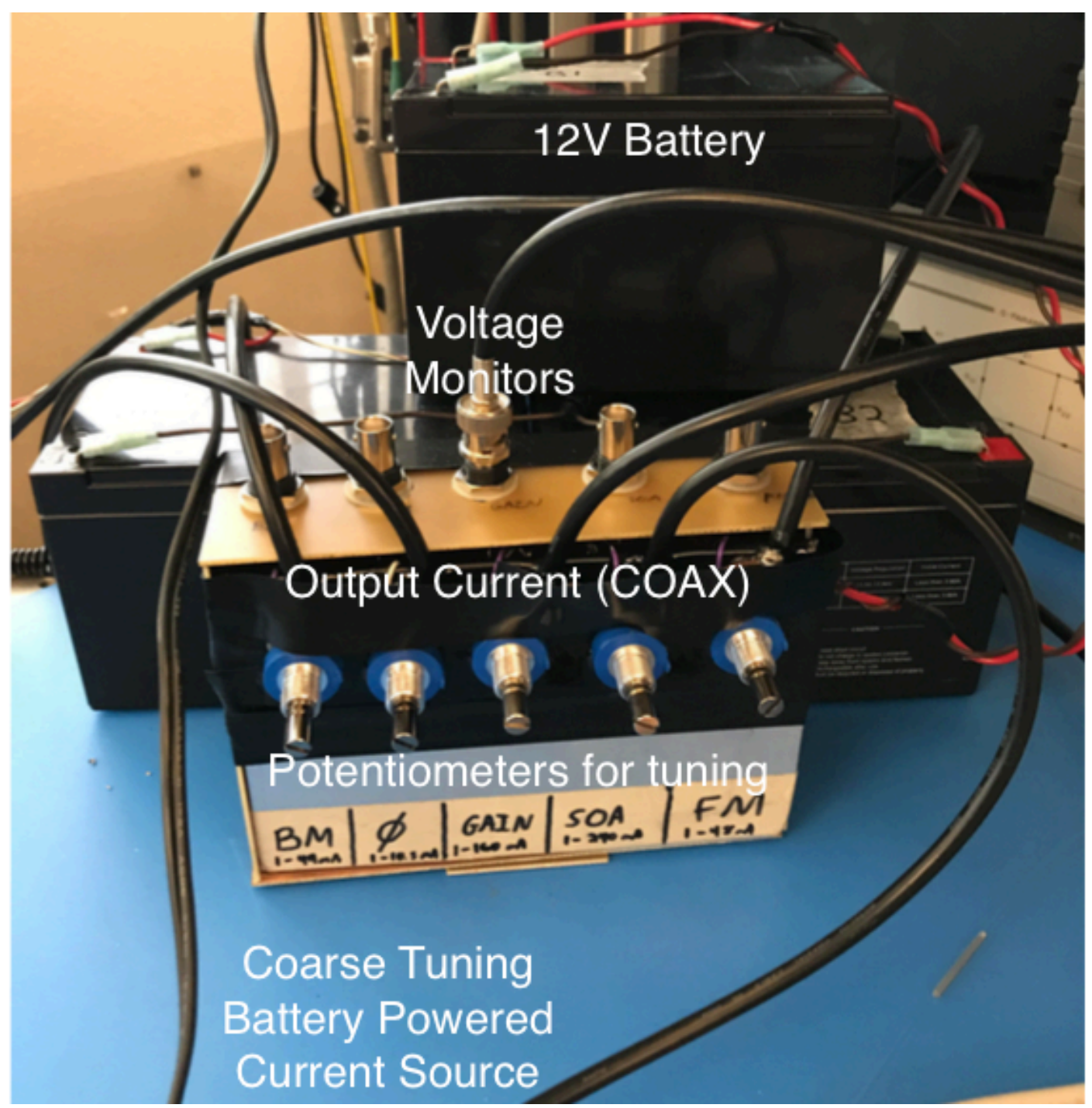

Figure 5.4: Full-range 12V battery powered current supply with SMA current outputs

The above current supply works well for biasing the laser up to its maximum current level for each section, but it lacks fine resolution in tuning and has a minimum output current of around $1 \mathrm{~mA}$. In order to explore biasing in the 100 s of $\mu \mathrm{A}$, a new supply was created. This small current supply works on the same principles at the large current supply, but with smaller batteries and different resistances. Figure 5.5 shows its circuit diagram and Figure 5.6 shows a built small current supply. Three of these supplies were built to bias each passive section of the laser independently. 


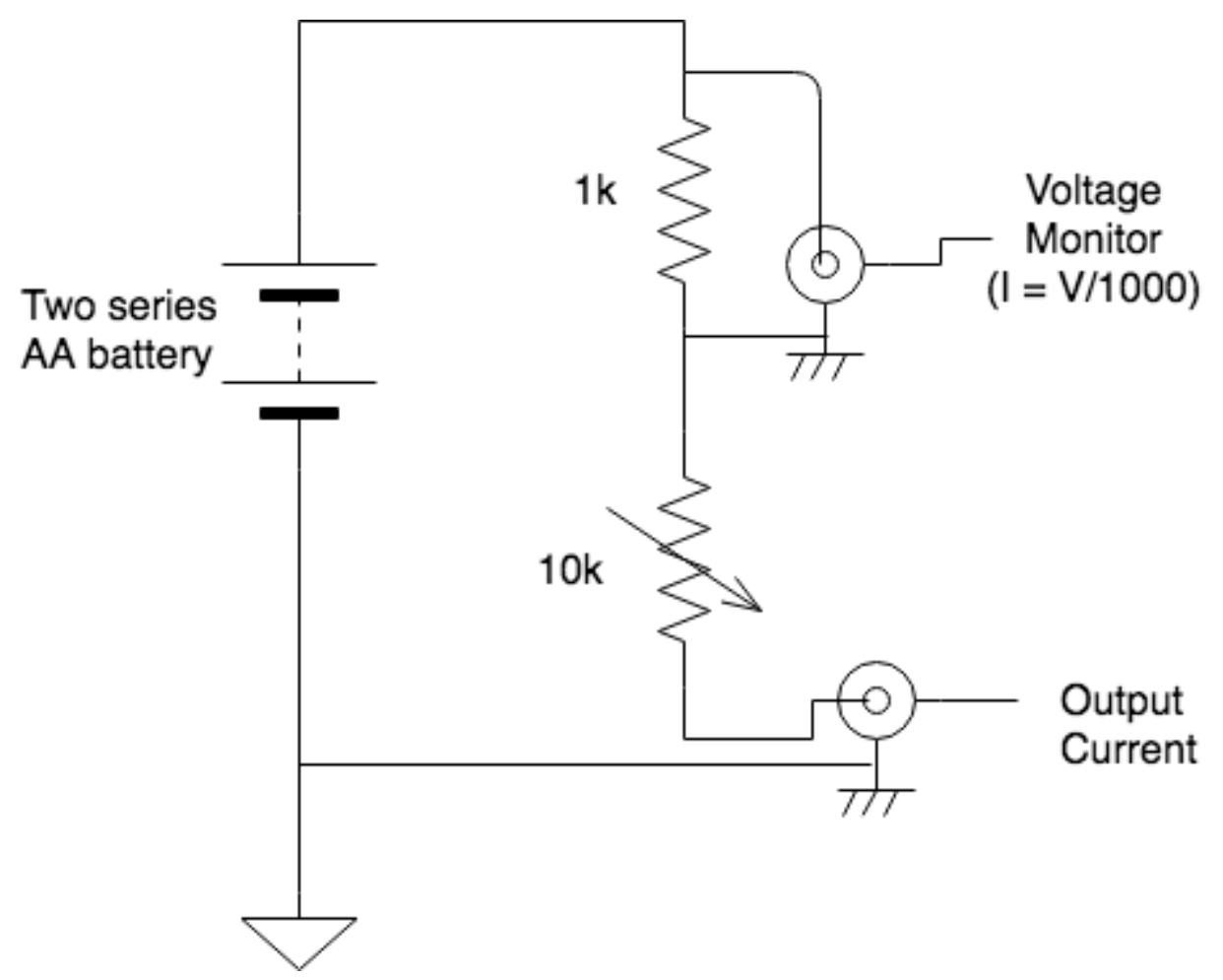

Figure 5.5: Circuit diagram for small-current battery powered current supply

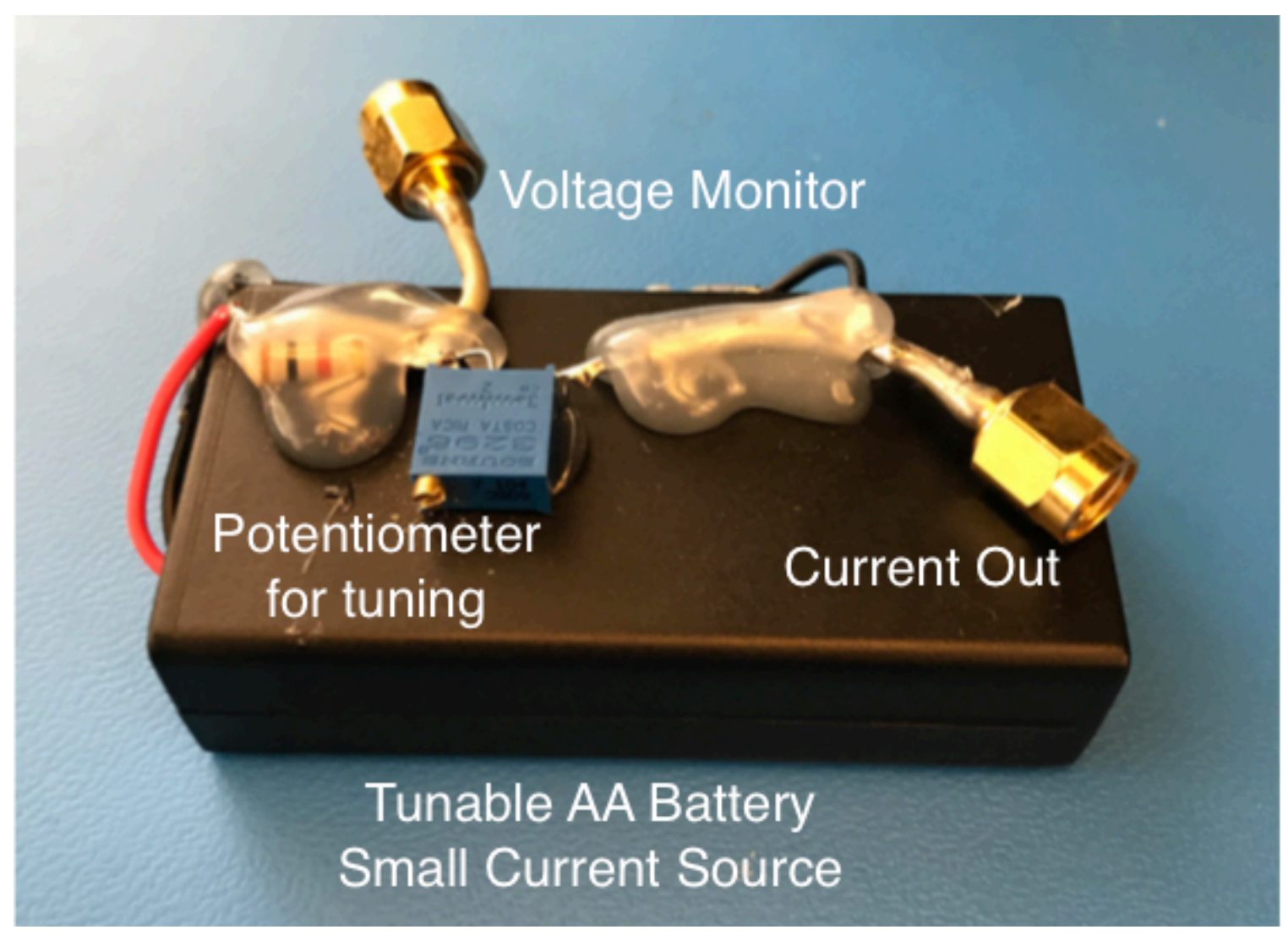

Figure 5.6: AA battery powered small current supply with semi-rigid coaxial output 
In order to explore the reverse bias behavior of the laser, a third current supply was designed to sink current from the laser as opposed to injecting current in the previous designs. This was accomplished using the ML-7905A negative voltage regulator powered by a 9V battery in reverse orientation. The regulated output voltage was adjustable and a $1 \mathrm{k} \Omega$ resistor limited the current. Figure 5.7 shows its circuit diagram and Figure 5.8 shows a built reverse current supply. Three of these supplies were built to bias each passive section of the laser independently.

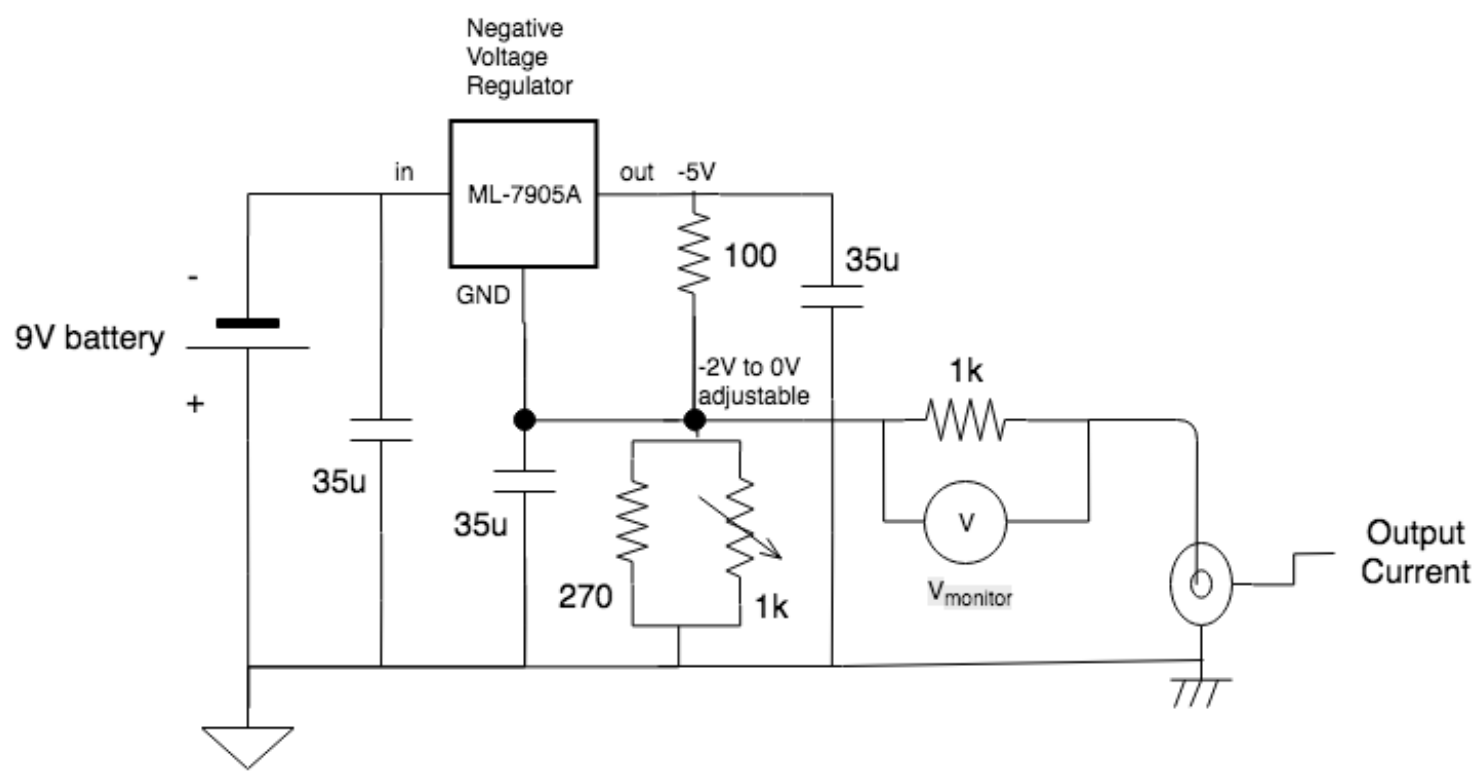

Figure 5.7: Circuit diagram for 9V battery powered reverse current supply using ML-7905A negative voltage regulator 


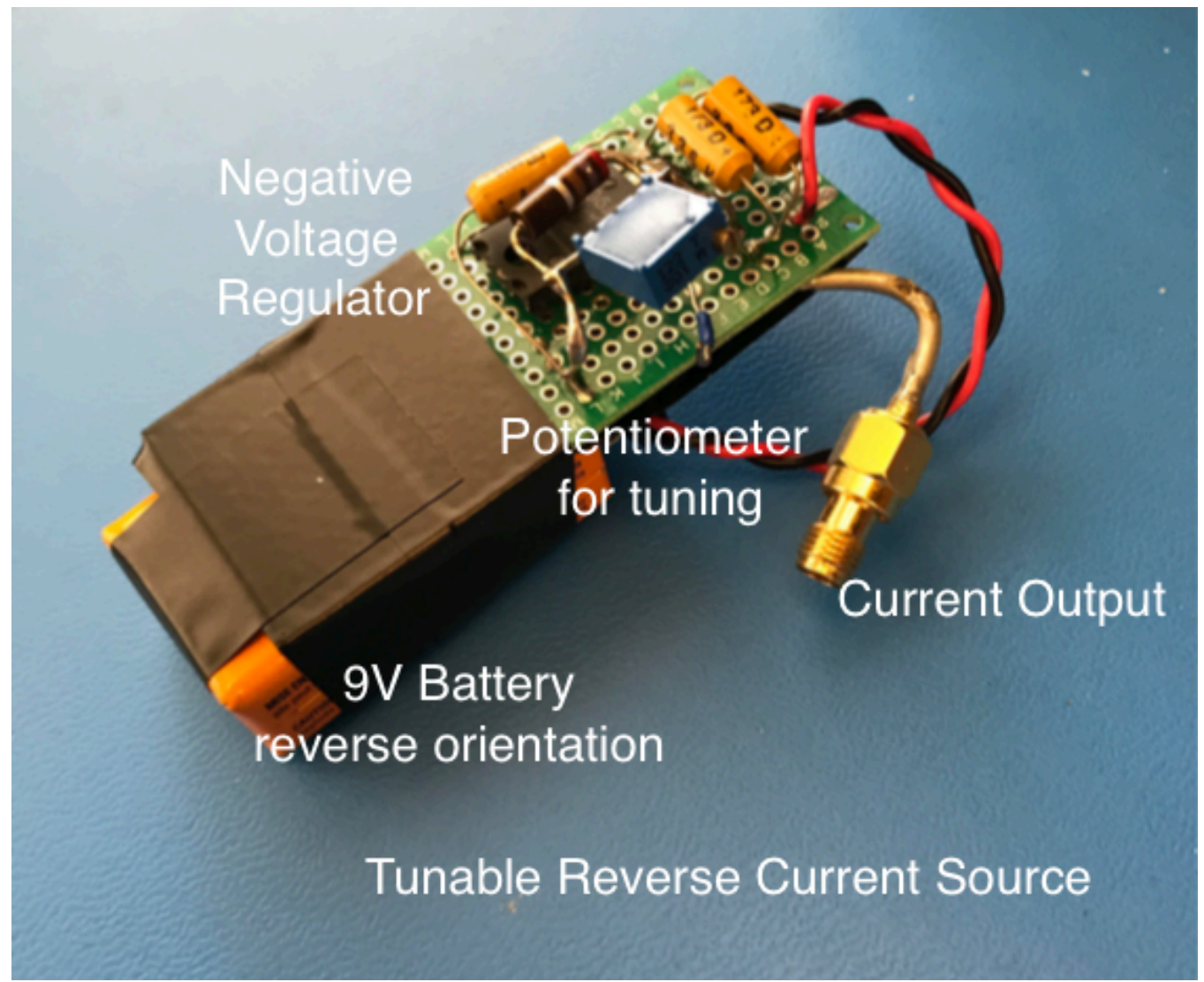

Figure 5.8: 9V battery powered reverse current supply with semi-rigid coaxial output

Finally, to explore laser behavior without any externally applied bias, two simple circuits were built to emulate a $50 \Omega$ termination resistor with a variable resistance. One is simply a potentiometer connected between the center conductor and ground of an SMA edge-launch connector, while the second performs the same function using an MPF102 JFET in the ohmic region as a voltage controlled resistor (VCR). Figure 5.9 shows the VCR circuit and 5.10 shows the two circuits side by side. 


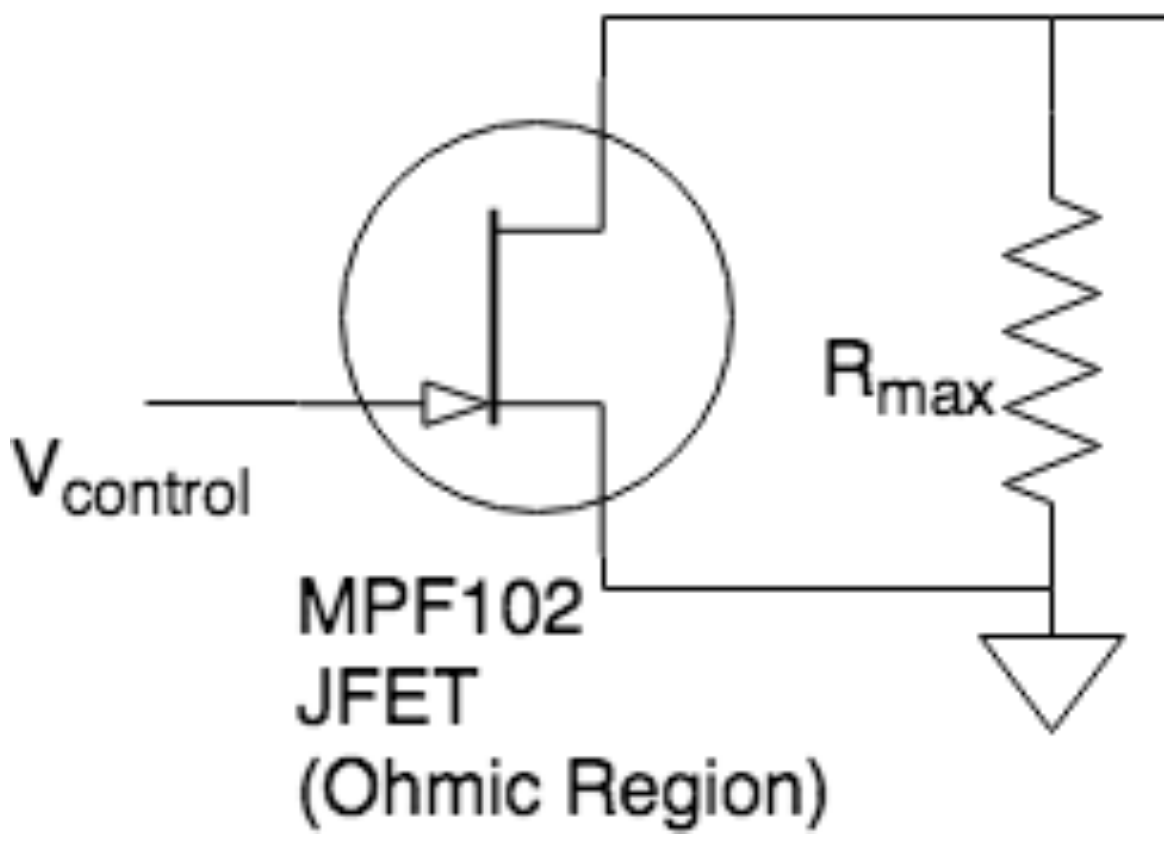

Figure 5.9: VCR circuit with MPF102 JFET

\section{0k Potentiometer}

with SMA connection Voltage Controlled Resistor

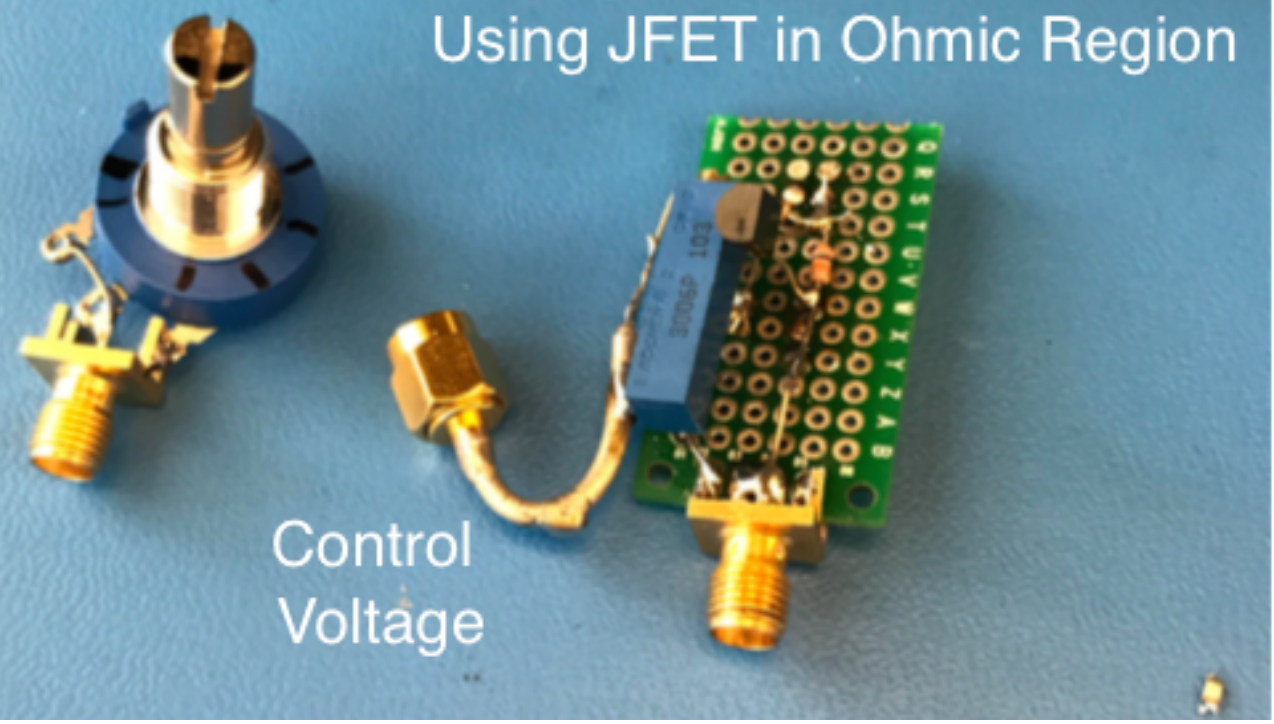

Figure 5.10: Variable termination resistors 


\subsection{Optical Circuit}

The optical circuit consisted of two distinct parts: a diagnostics section and a feedback section.

The diagnostic section gives a measurement of laser spectrum, changes in optical power, and an estimate of the laser linewidth. This linewidth estimate is discussed in the next section. The feedback section consists of a Mach-Zehnder interferometer with a free spectral range (FSR) of $2 \mathrm{GHz}$ and fiber leads shortened to just a few $\mathrm{cm}$. The outputs of the interferometer are then detected using a balanced photo-detector, whose RF output is now proportional to small differences in laser frequency. This signal is the error signal of the optoelectronic feedback control loop. It is then input to an integrator on the laser board and fed back to the laser phase section. A schematic block diagram of the optical circuit is shown in Figure 5.11.

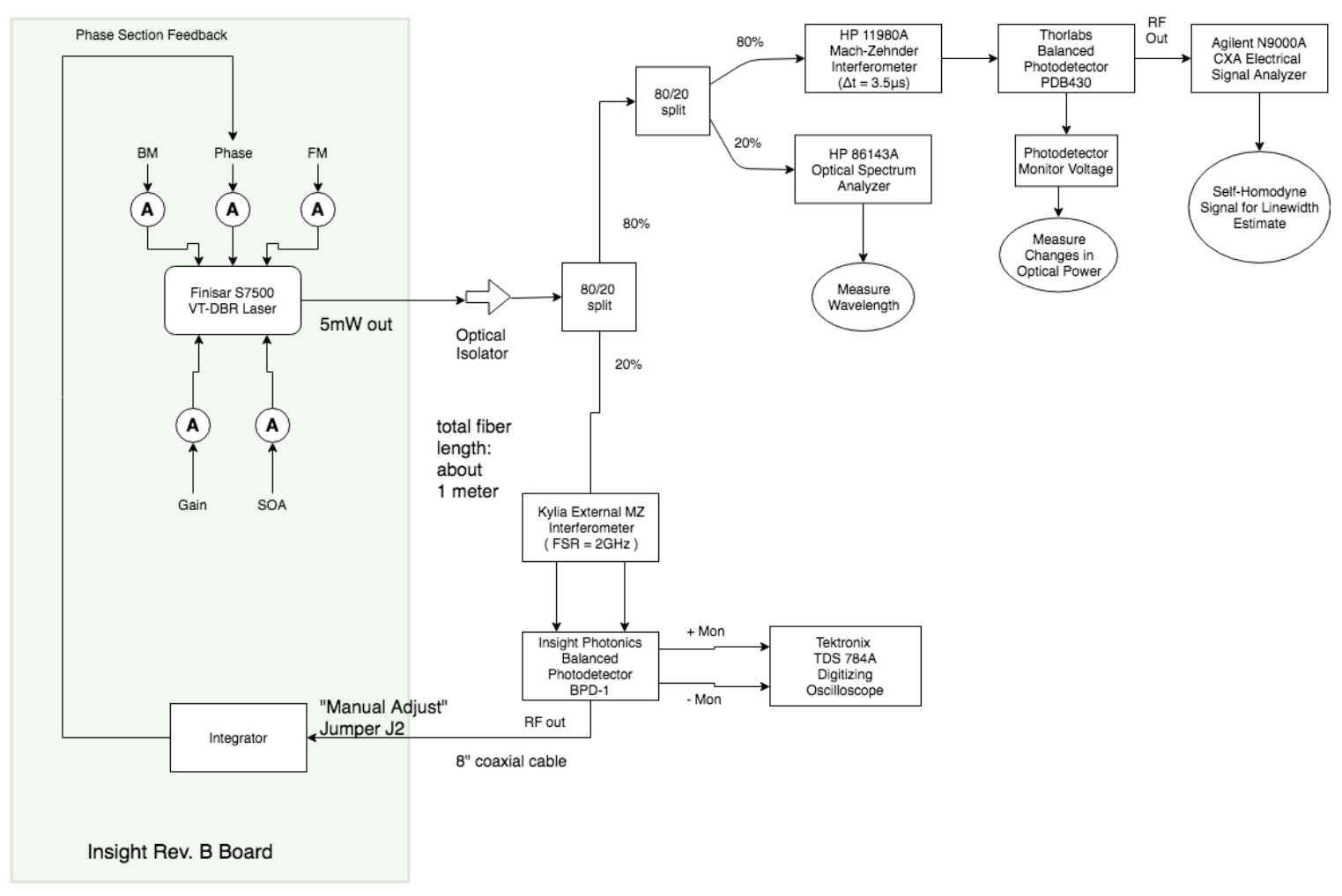

Figure 5.11: Block diagram for optical circuit showing diagnostic section (upper right) and feedback section (lower left) 


\subsection{Self-Homodyne Linewidth Estimation}

Measuring the laser linewidth accurately is impossible using most optical spectrum analyzers (OSAs) because they lack the measurement resolution to see laser linewidth. For this reason, a selfhomodyne linewidth measurement is used to estimate the laser linewidth. This method uses the fact that the interference between two incoherent sources causes intensity variations that are a function of phase variations caused by differences in laser frequency. The self-homodyne measurement can be thought of as a single-delay autocorrelation of the optical spectrum, where the original signal is mixed with a time-delayed copy of itself. This mixed signal is then detected on a fast photodiode, and the output of this photodiode is analyzed on a spectrum analyzer. The signal will be the positive part of a nearly Lorentzian lineshape centered at $0 \mathrm{~Hz}$, and the $-3 \mathrm{~dB}$ bandwidth of this signal is the estimate of the laser linewidth. The timedelayed mixing and the resulting spectrum are shown in Figure 5.12 [11] This effect is explained in detail in Section 4.4 and can be seen in Figure 4.3.

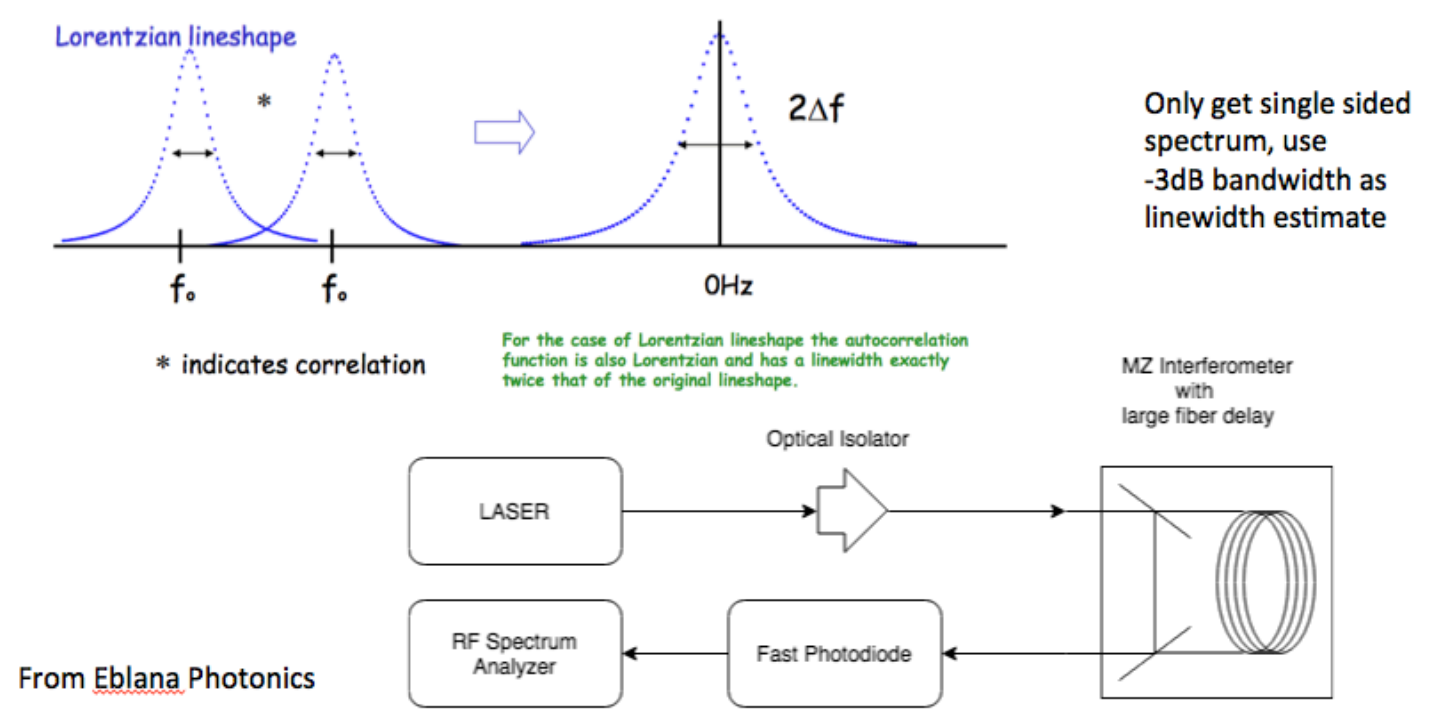

Figure 5.12: Block diagram for self-homodyne linewidth estimation, with corresponding spectrum after mixing. [11] 


\section{Chapter 6: Biasing for Narrow Linewidth}

\subsection{Full Scale Forward Biasing}

Using the $12 \mathrm{~V}$ battery powered current supply shown in Figures 5.3 and 5.4, the S7500 laser could be tuned through its entire range of frequencies (Figure 3.10 region A) by adjusting the potentiometers for each of the front mirror, back mirror, and phase sections. With the mirror sections biased with more than $1 \mathrm{~mA}$, the linewidth increases from its intrinsic value of a few $\mathrm{MHz}$ to hundreds of $\mathrm{MHz}$. This is shown in

Figure 6.1.

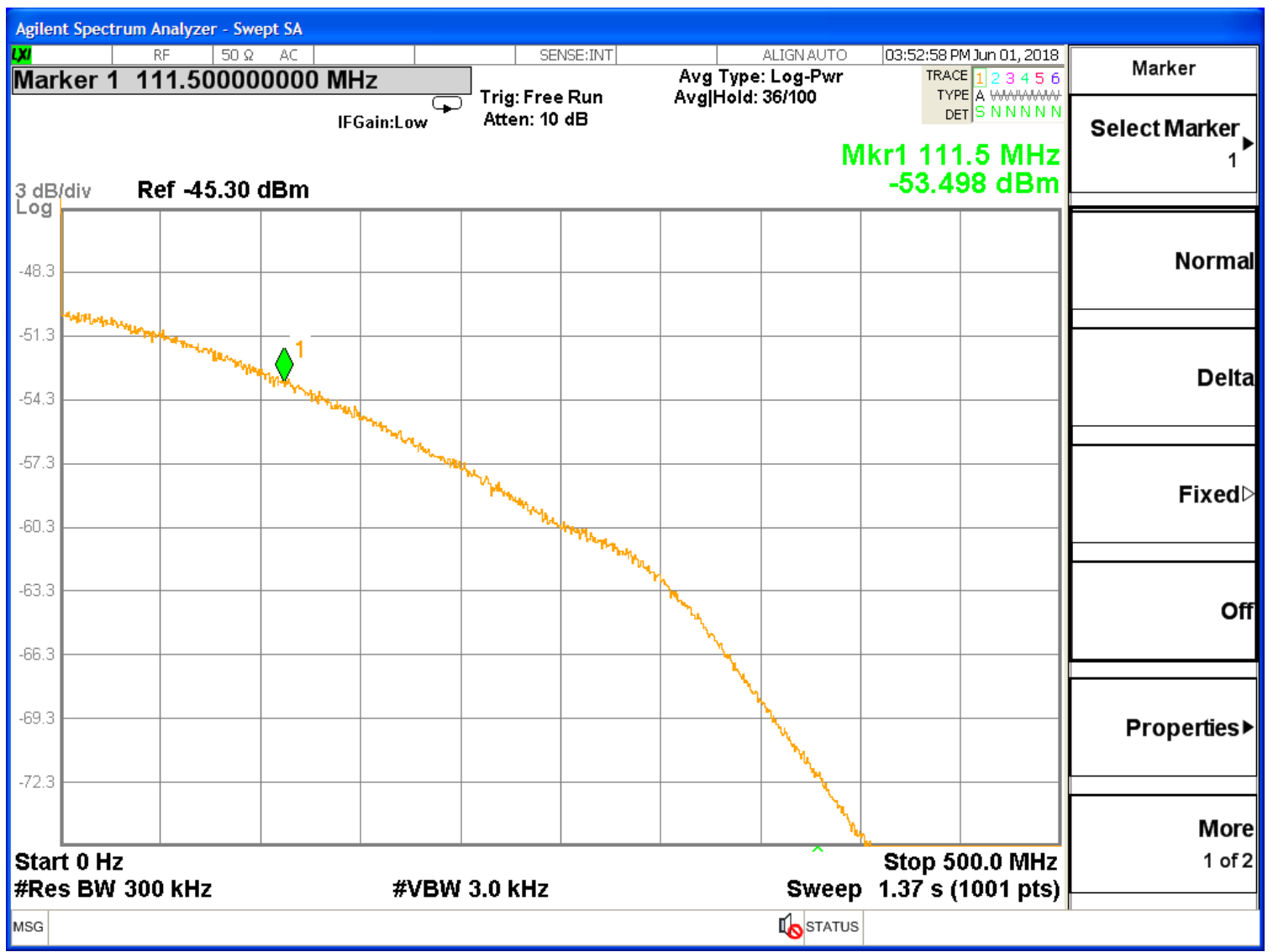

Figure 6.1: Self-Homodyne signal for all three passive sections forward biased. Broad $112 \mathrm{MHz}$ linewidth caused by current injection into both front and back mirrors

This linewidth broadening renders the laser useless for LIDAR at appreciably large ranges, and is the motivation for using currents less than $1 \mathrm{~mA}$ to bias the passive sections.

\subsection{Small Current Forward Biasing}

If the passive sections are instead biased within region B from Figure 3.10 using the small current supply shown in Figures 5.5 and 5.6 an interesting observation can be made. Almost the entire range of 
laser frequencies can be tuned through while keeping the injected current under $1 \mathrm{~mA}$ into each section. The laser linewidth was still seen to be tens to hundreds of MHz for most bias conditions with small currents, but there existed small pockets of intrinsically narrow linewidth. However, these narrow linewidth bias conditions were sparse and even a small deviation from that bias causes a large increase in linewidth. Figures 6.2 through 6.4 show the wavelength, linewidth, and changes in optical power for each section biased independently. For these measurements, the two sections not under test are terminated with $50 \Omega$ to ground.

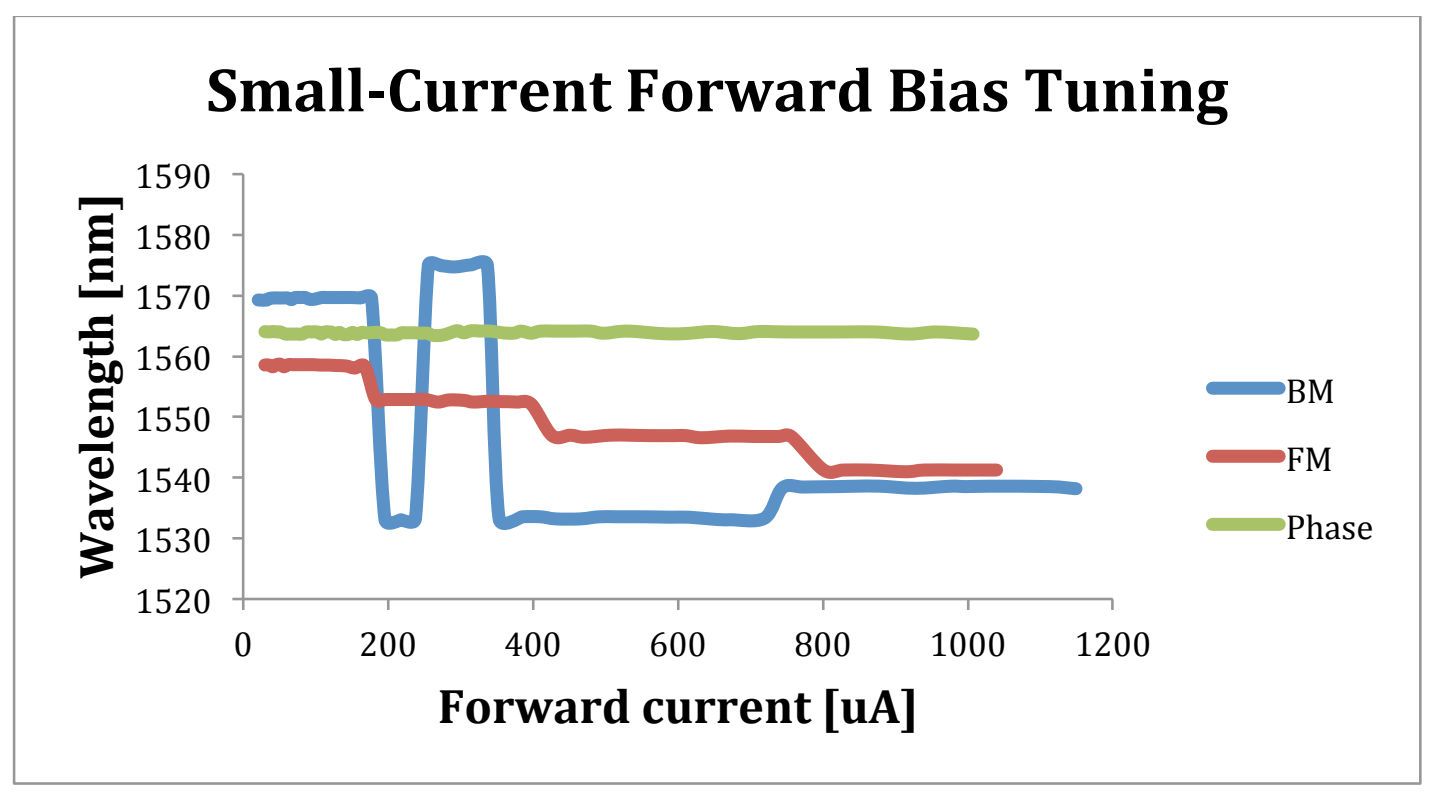

Figure 6.2: Independent section small-current forward bias wavelength tuning. A large portion of the full wavelength tuning range is covered with currents less than $1 \mathrm{~mA}$. 

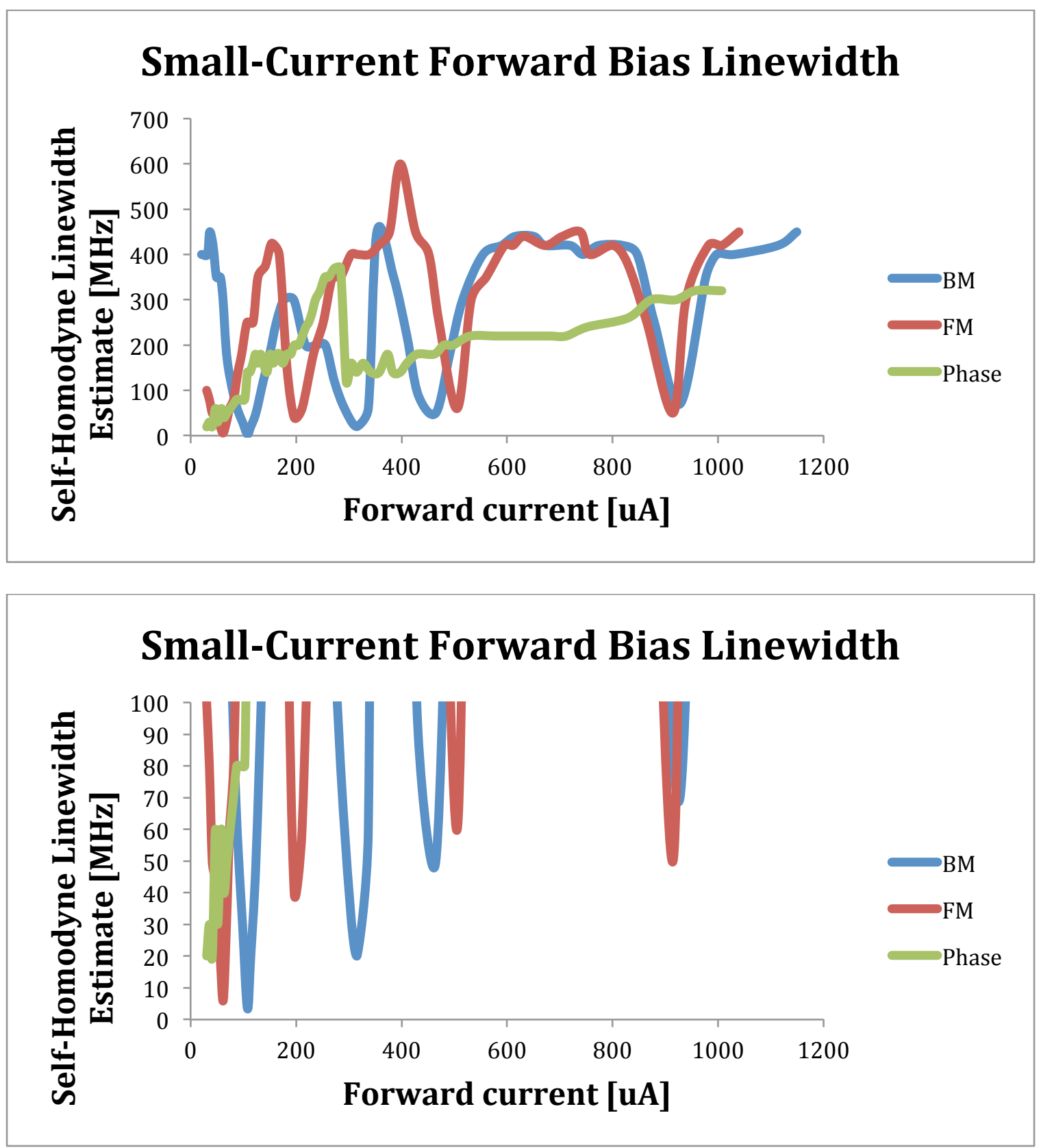

Figure 6.3: Independent section small-current forward bias linewidth. Top plot shows the full range of linewidth while the bottom plot shows the regions of linewidth under $100 \mathrm{MHz}$. It can be seen that there are some bias conditions with $>10 \mathrm{MHz}$ linewidth. 


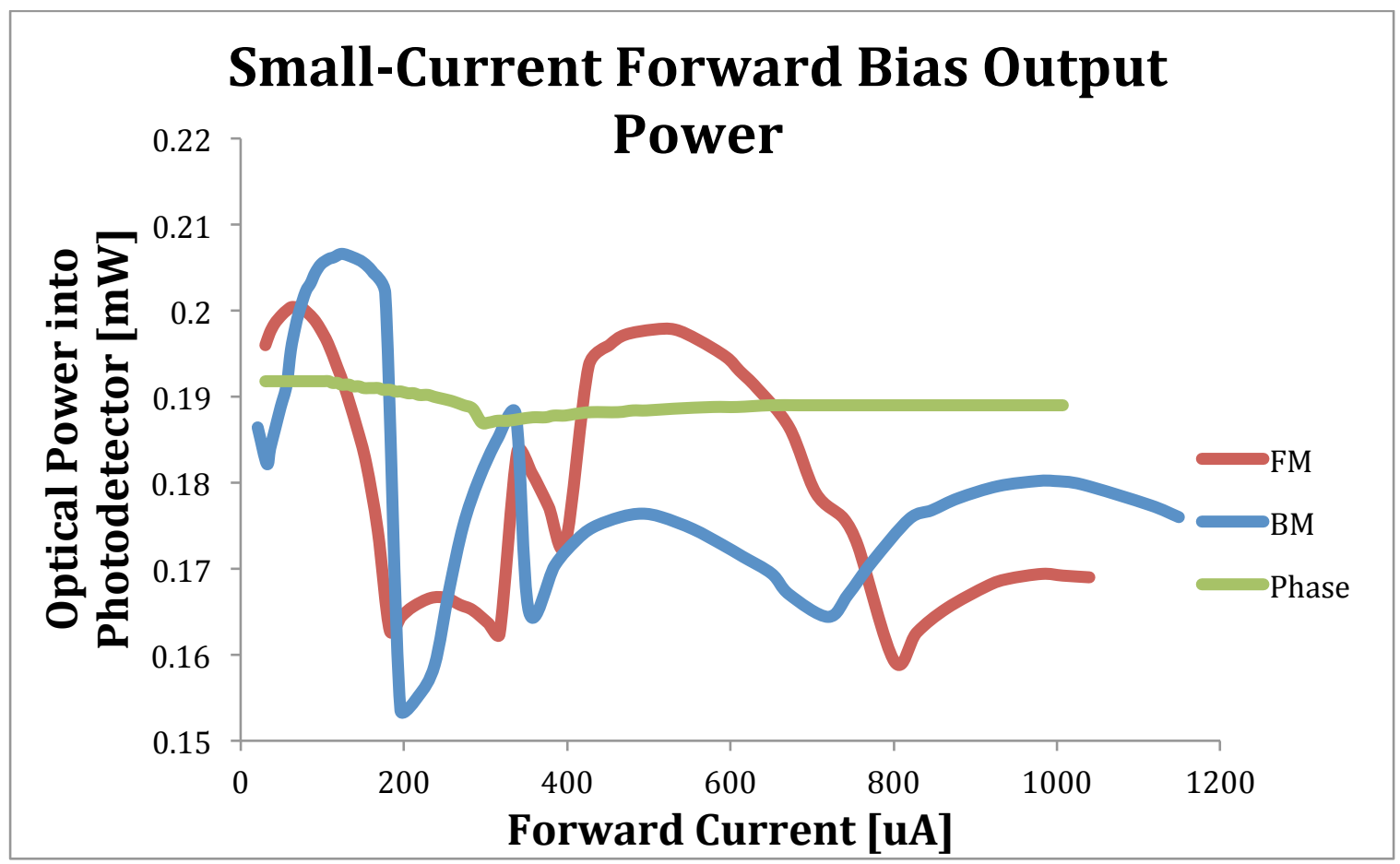

Figure 6.4: Independent section small-current forward bias output power variation

While independent section tests have some use, more interesting is a mapping of laser behavior as a function of all three currents simultaneously. The maps were created by measuring the wavelength and linewidth in a grid spaced $0.1 \mathrm{~mA}$ by $0.1 \mathrm{~mA}$ for front and back mirror currents. The data points are marked with a red ' $x$ ', and then interpolated using MATLAB to create the surfaces below. Figures 6.5 and 6.6 both show a wavelength map over front and back mirror currents from 0 to $1 \mathrm{~mA}$, with the phase section grounded in Figure 6.5 and phase current equal to $1 \mathrm{~mA}$ in Figure 6.6. 

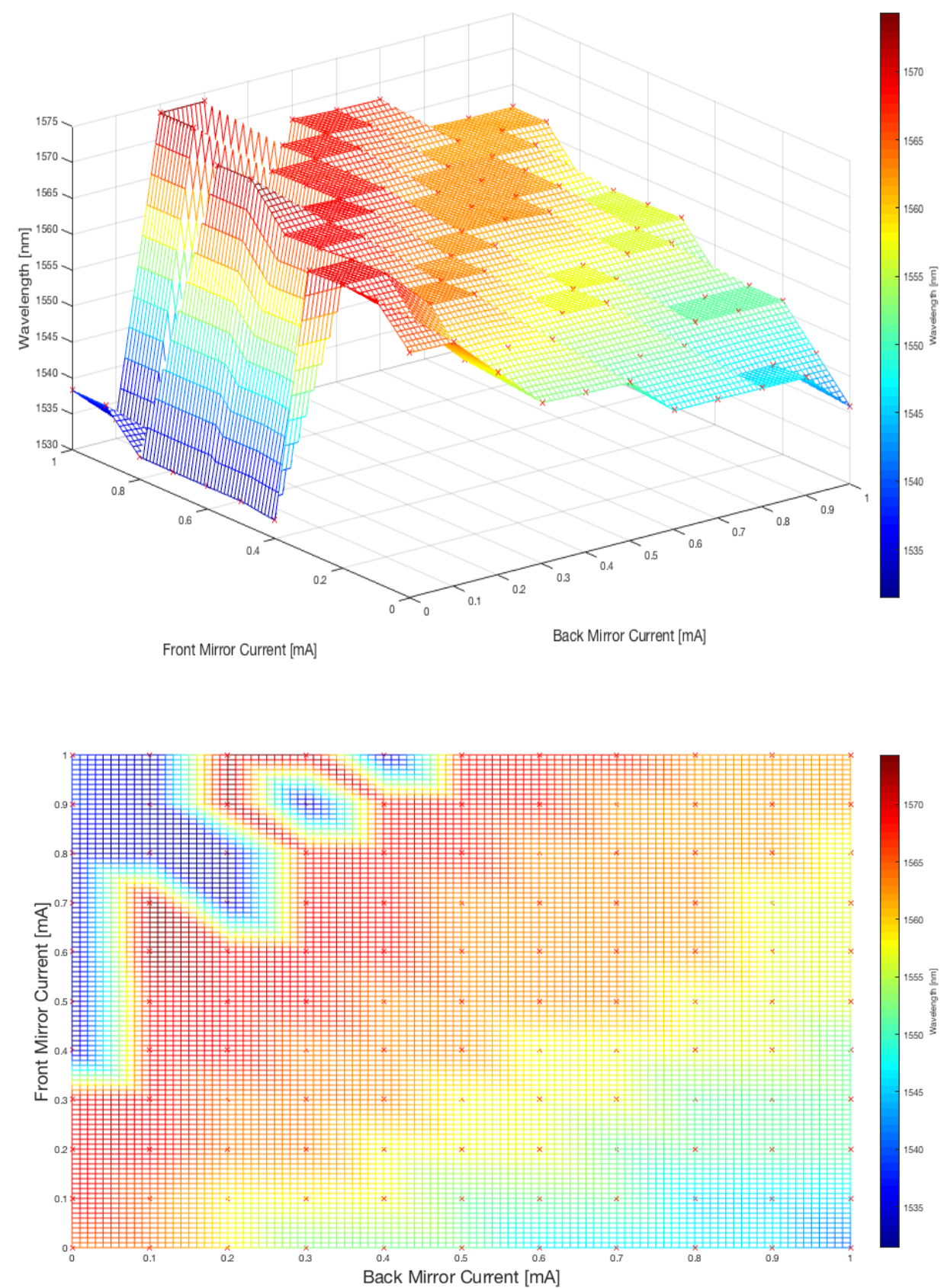

Figure 6.5: Wavelength map over FM and BM currents from 0 to $1 \mathrm{~mA}$. Phase section was grounded for this measurement. This map shows large scale wavelength tuning is possible with currents less than $1 \mathrm{~mA}$. 

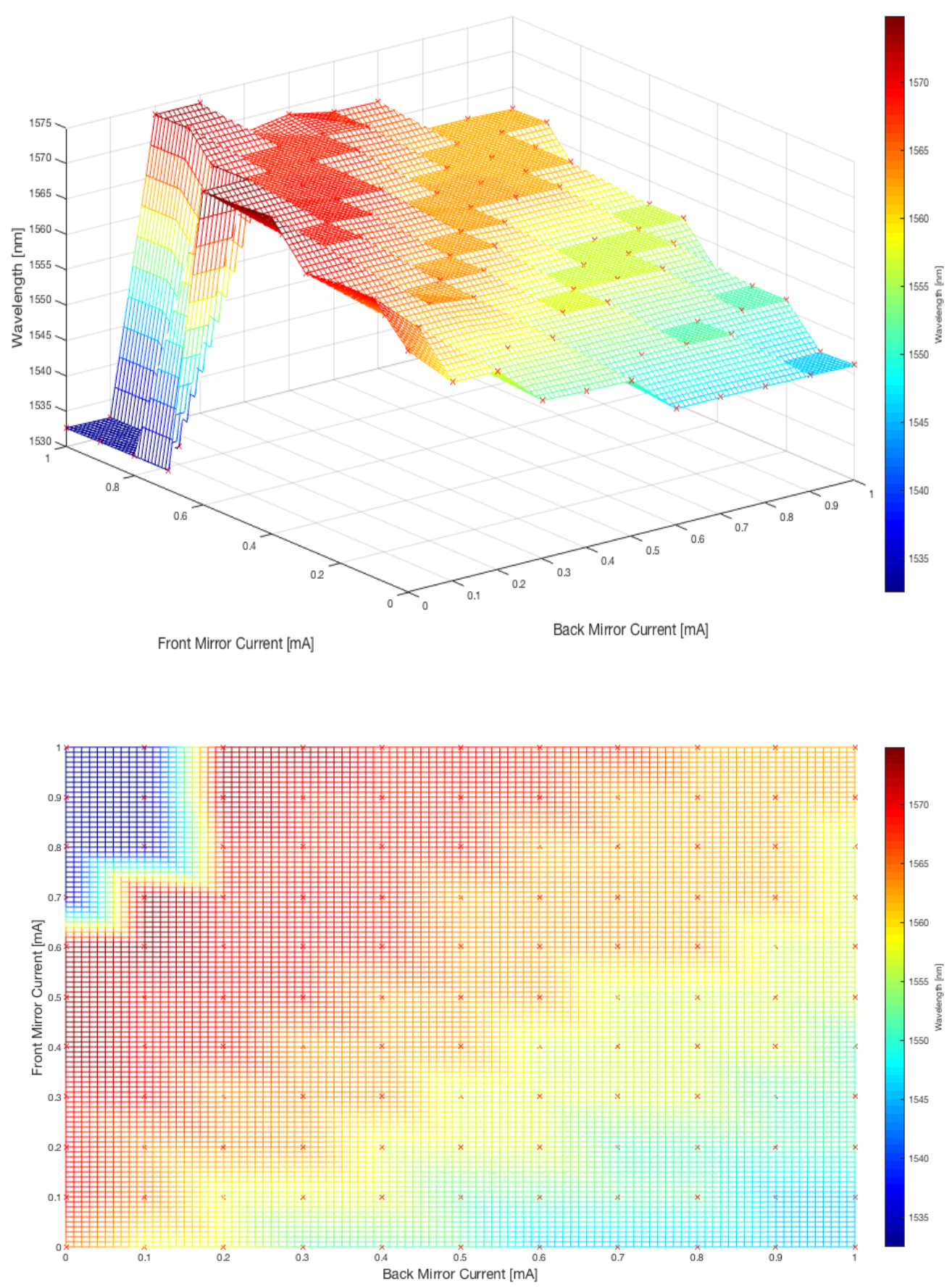

Figure 6.6: Wavelength map over FM and BM currents from 0 to $1 \mathrm{~mA}$. Phase current is $1 \mathrm{~mA}$ for this measurement. The effect of the additional phase current can be seen to decrease the wavelength over then entire space 
Figures 6.5 and 6.5 show tuning currents less than $1 \mathrm{~mA}$ cover a large part of the laser tuning range. The effect of the addition of phase current is seen in Figure 6.6 to shift the wavelength along each tuning path to the blue without much effect on the overall shape of the paths. The linewidth maps for each of these situations are shown in Figures 6.7 and 6.8. The addition of $1 \mathrm{~mA}$ phase current in Figure 6.8 is seen to increase the linewidth for most combinations of FM and BM currents.

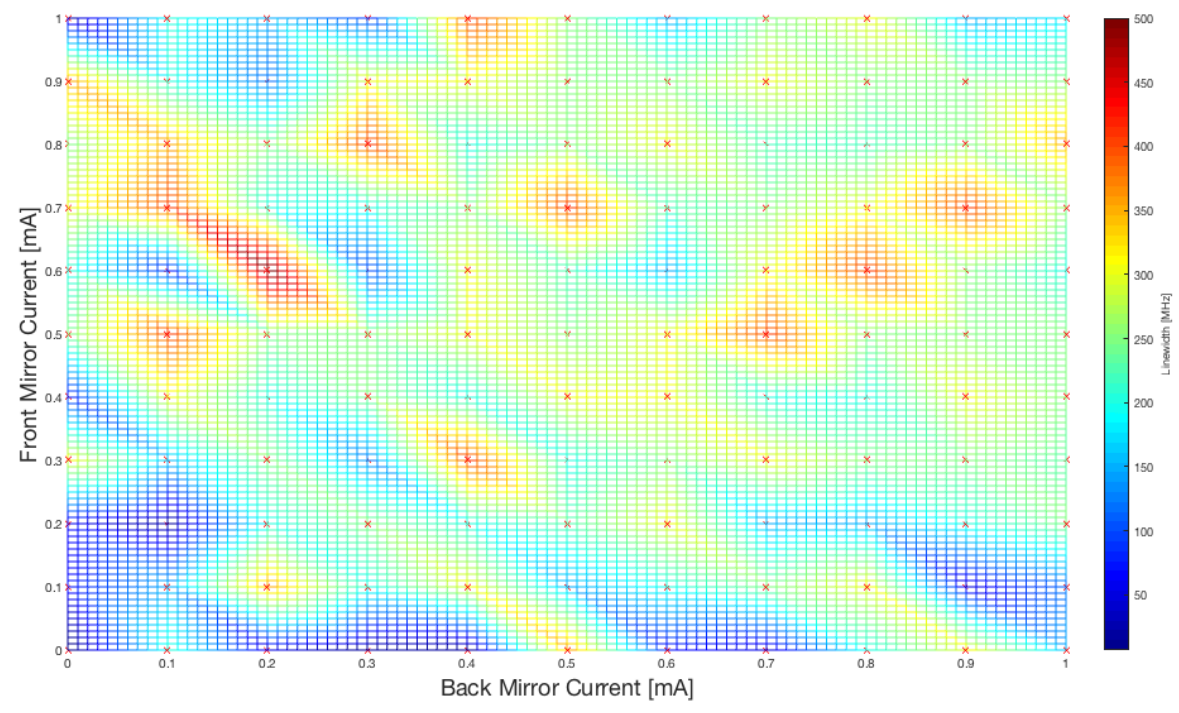

Figure 6.7: Linewidth map over FM and BM currents from 0 to $1 \mathrm{~mA}$. Phase section is grounded for this measurement. There are pockets of narrow linewidth but a finer mapping of this space is needed to determine the range of wavelengths with narrow linewidth. 


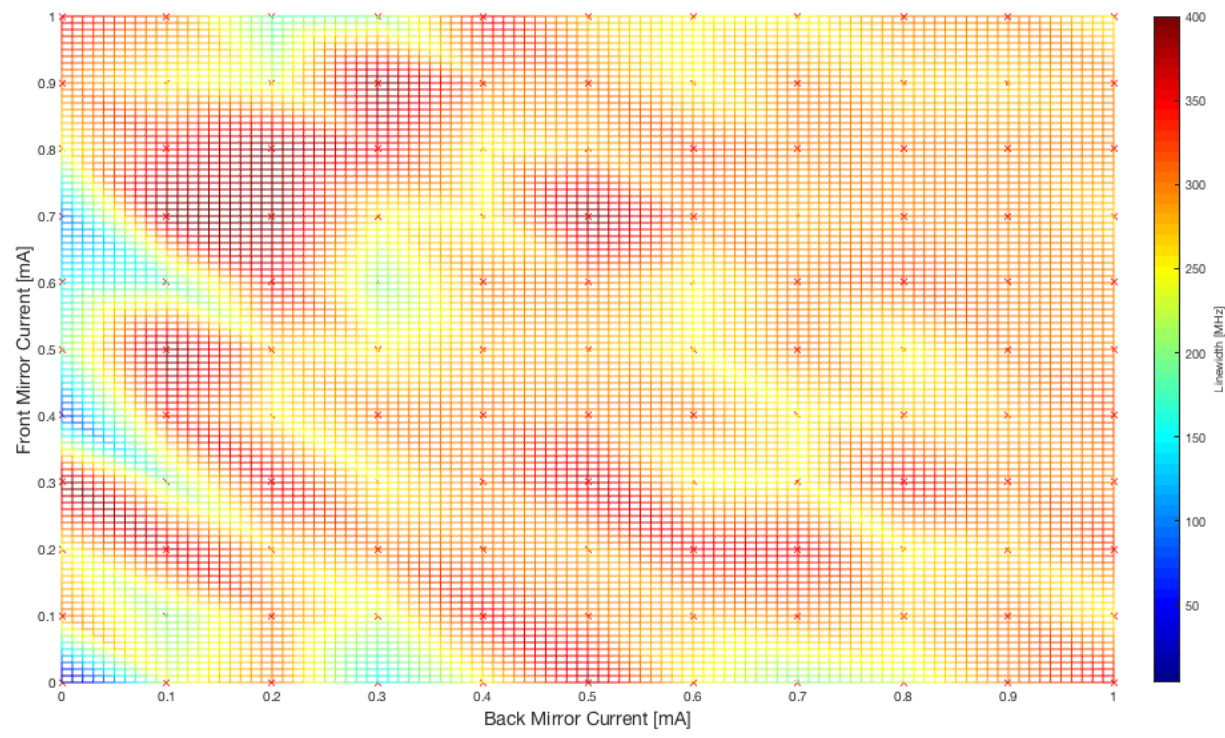

Figure 6.8: Linewidth map over FM and BM currents from 0 to $1 \mathrm{~mA}$. Phase current is $1 \mathrm{~mA}$ for this measurement. The addition of phase current injection is seen to increase the linewidth for most bias conditions.

\subsection{Reverse Biasing}

Reverse basing in region C from Figure 3.10 using the supply shown in Figures 5.7 and 5.8 leads to interesting results. The tuning range becomes limited to a few hundred pm around $1564 \mathrm{~nm}$, but each independent section has a similar and nearly linear tuning curve. Additionally, the laser linewidth was much narrower in general but there still existed pockets of larger linewidth. Figures 6.9 to 6.11 show the wavelength, linewidth, and changes in optical power for each section biased independently. For these measurements, the two sections not under test are terminated with $50 \Omega$ to ground. 


\section{Reverse Bias Wavelength Tuning}

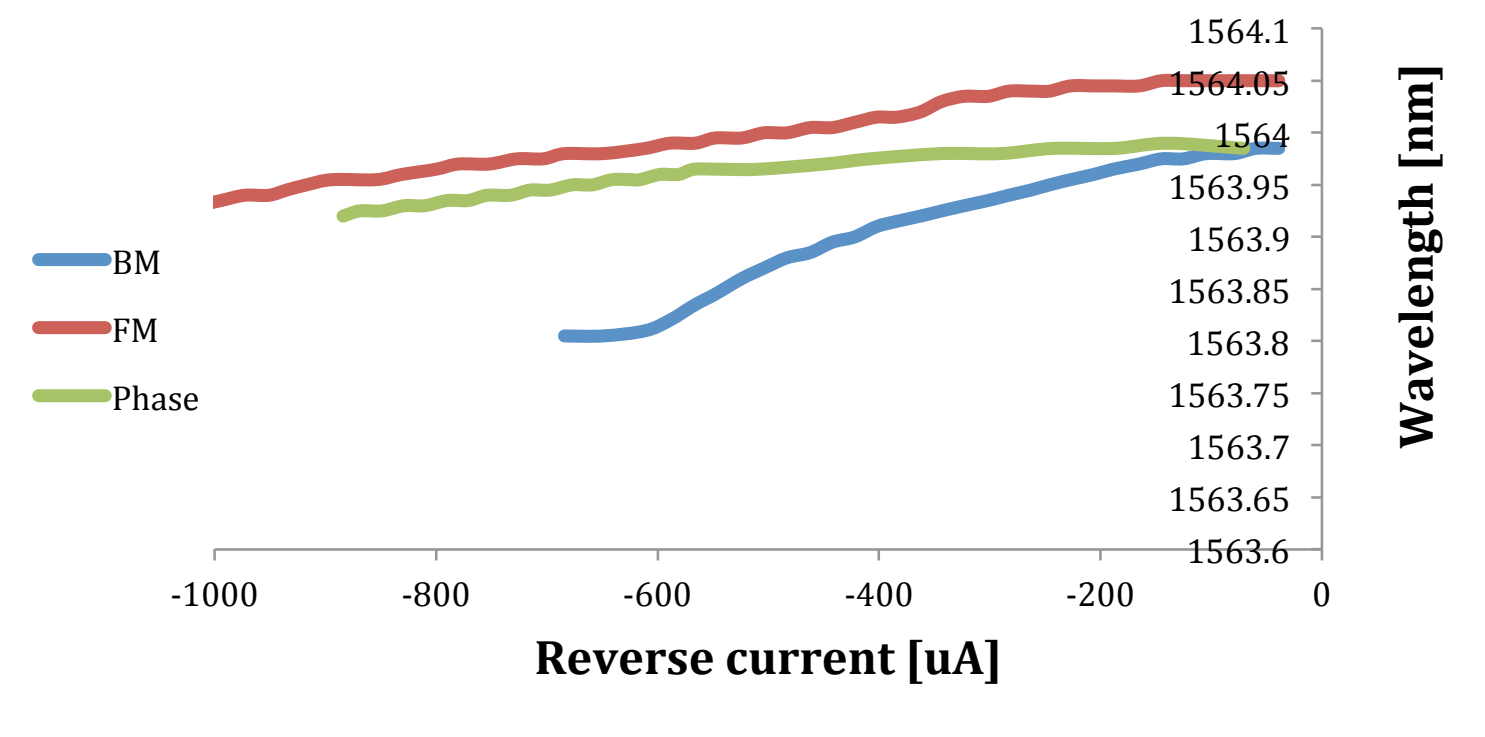

Figure 6.9: Independent section reverse bias wavelength tuning. Each section exhibits narrow but continuous wavelength tuning, with increased tuning capabilities in the back mirror section.

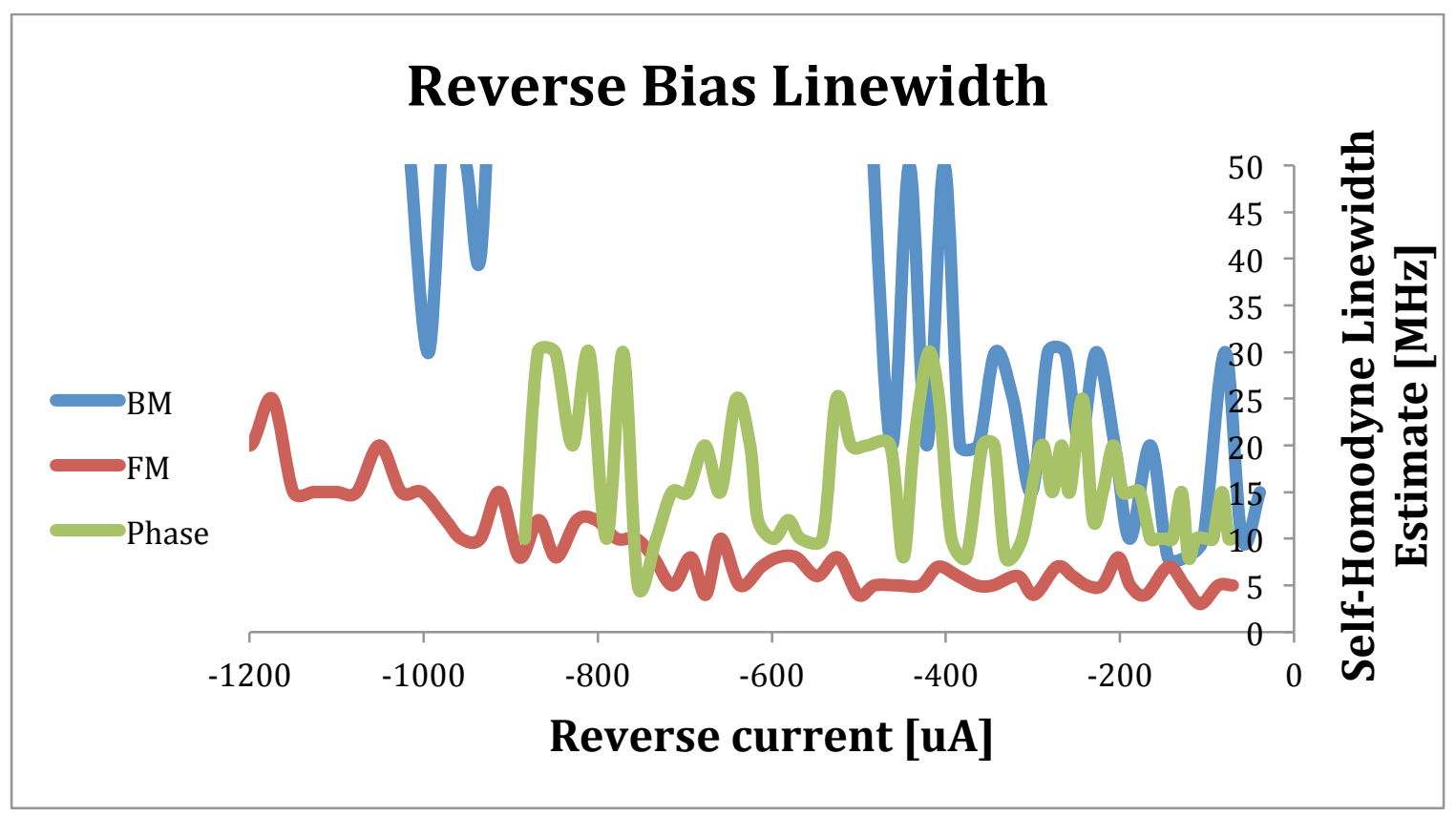

Figure 6.10: Independent section reverse bias linewidth. Independent front mirror tuning exhibits narrow linewidth but independent back mirror tuning is seen to exhibit increased linewidth. 


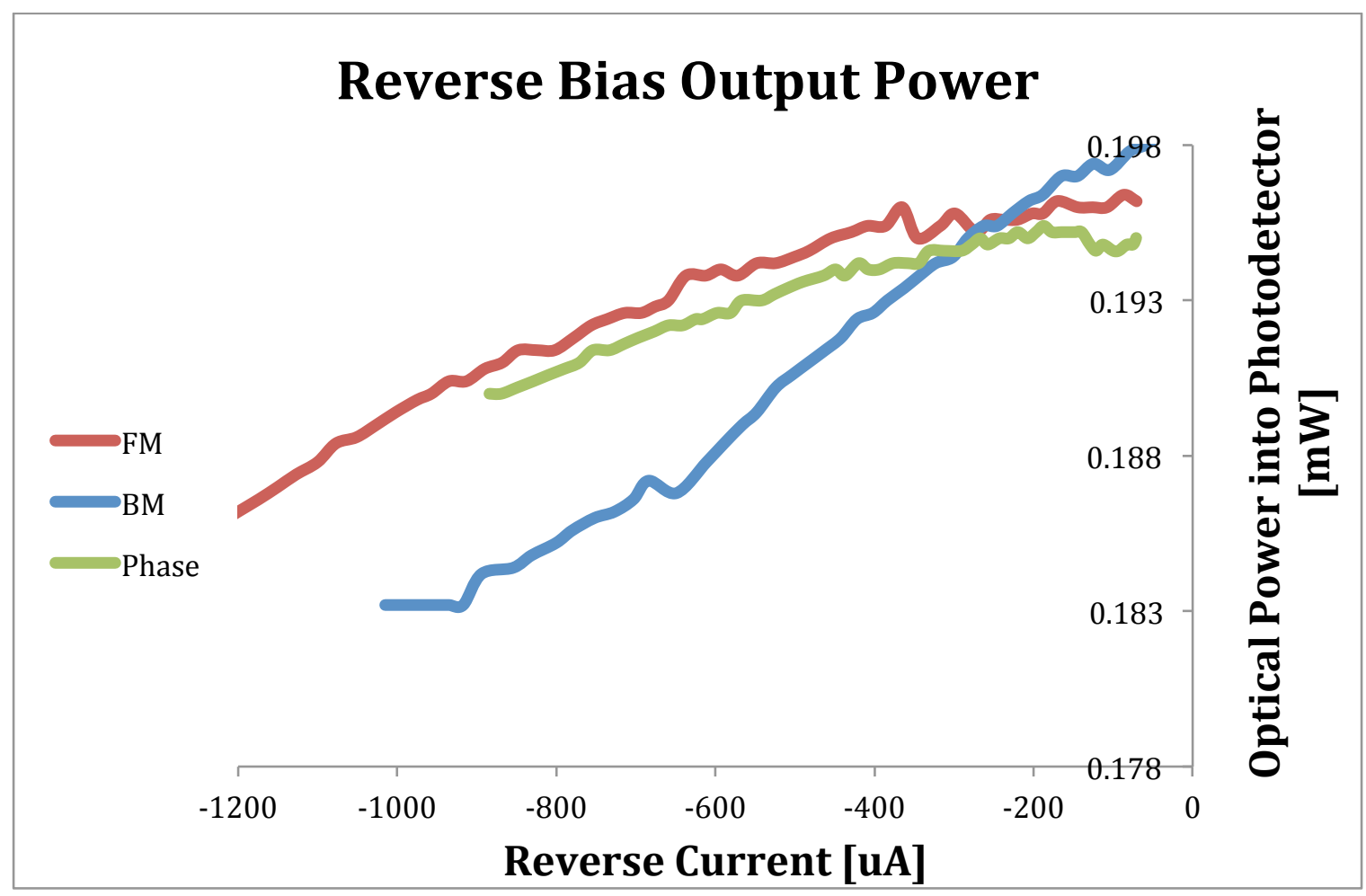

Figure 6.11: Independent section reverse bias output power variation. Increased current magnitude leads to a decrease in output optical power, with a greater decrease in power exhibited by the back mirror section.

This reverse bias space was mapped similarly to the forward bias space shown in Figures 6.5 to 6.8. Figure 6.12 shows the wavelength map and Figure 6.13 shows the linewidth map for a grounded phase section. It should be noted that the overall tuning range and linewidth is much lower than the forward bias case. 

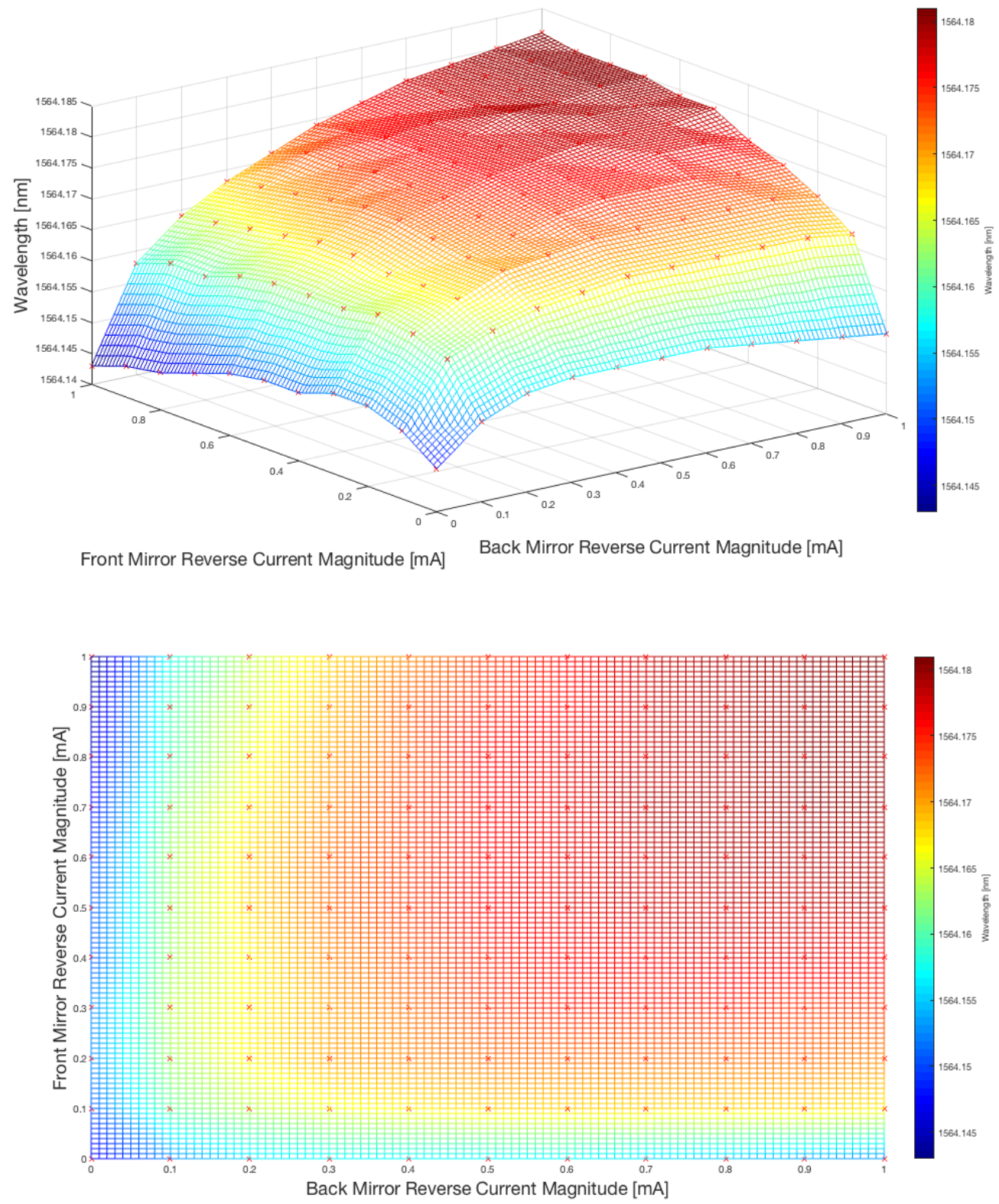

Figure 6.12: Reverse current wavelength map. Phase section grounded. Reverse biasing of the mirror sections leads to a smooth tuning surface with a 40 picometer tuning range. 


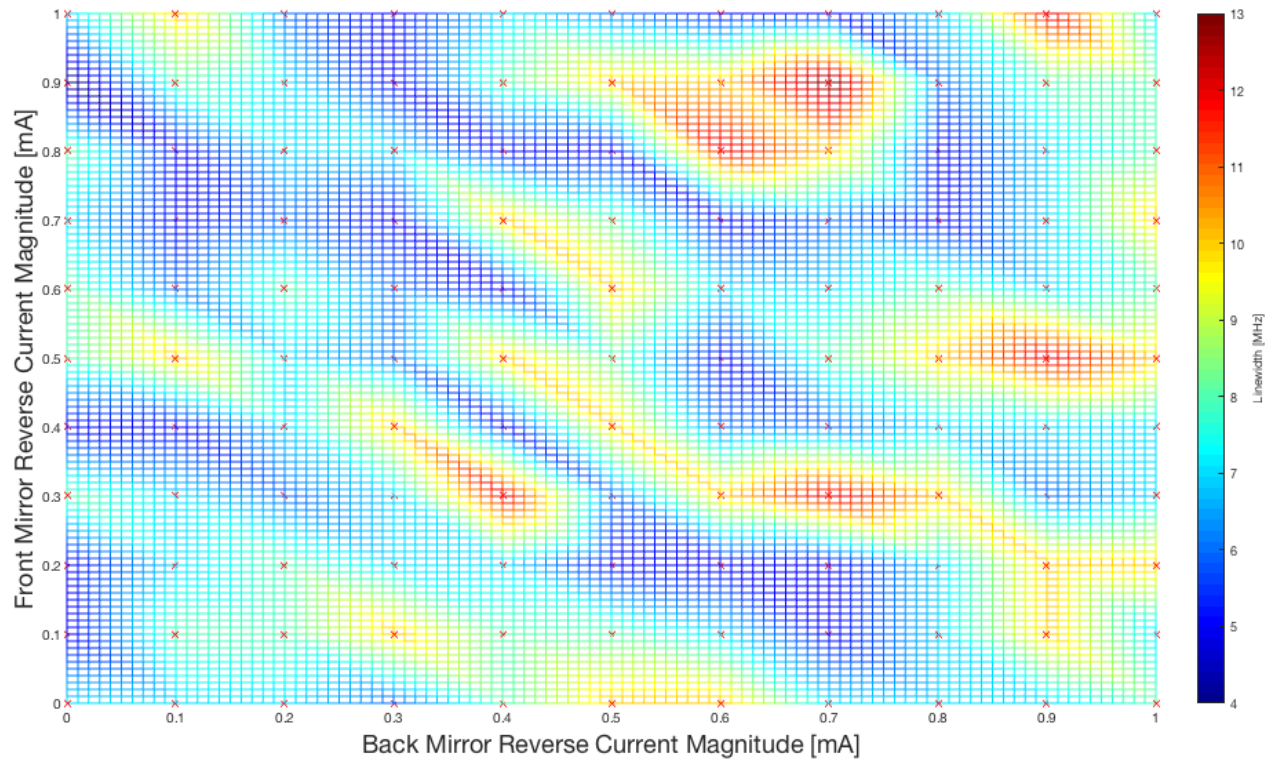

Figure 6.13: Reverse current linewidth map. Phase section grounded. Reverse biasing of the mirror sections leads to narrower linewidth compared to forward biasing. This plot shows apparent paths of narrow linewidth

While the reverse bias linewidth map shows that this entire range exhibits narrower-than-usual linewidth, there are sections of the map with particularly narrow linewidth near 4-5MHz. Some of these apparent tuning paths are shown on both the linewidth map and the wavelength map in Figure 6.14. These narrow-linewidth paths are seen to vary in tuning range, with path $\mathrm{C}$ giving a change in wavelength of $30 \mathrm{pm}$ and path A giving a change in wavelength of less than $5 \mathrm{pm}$. 

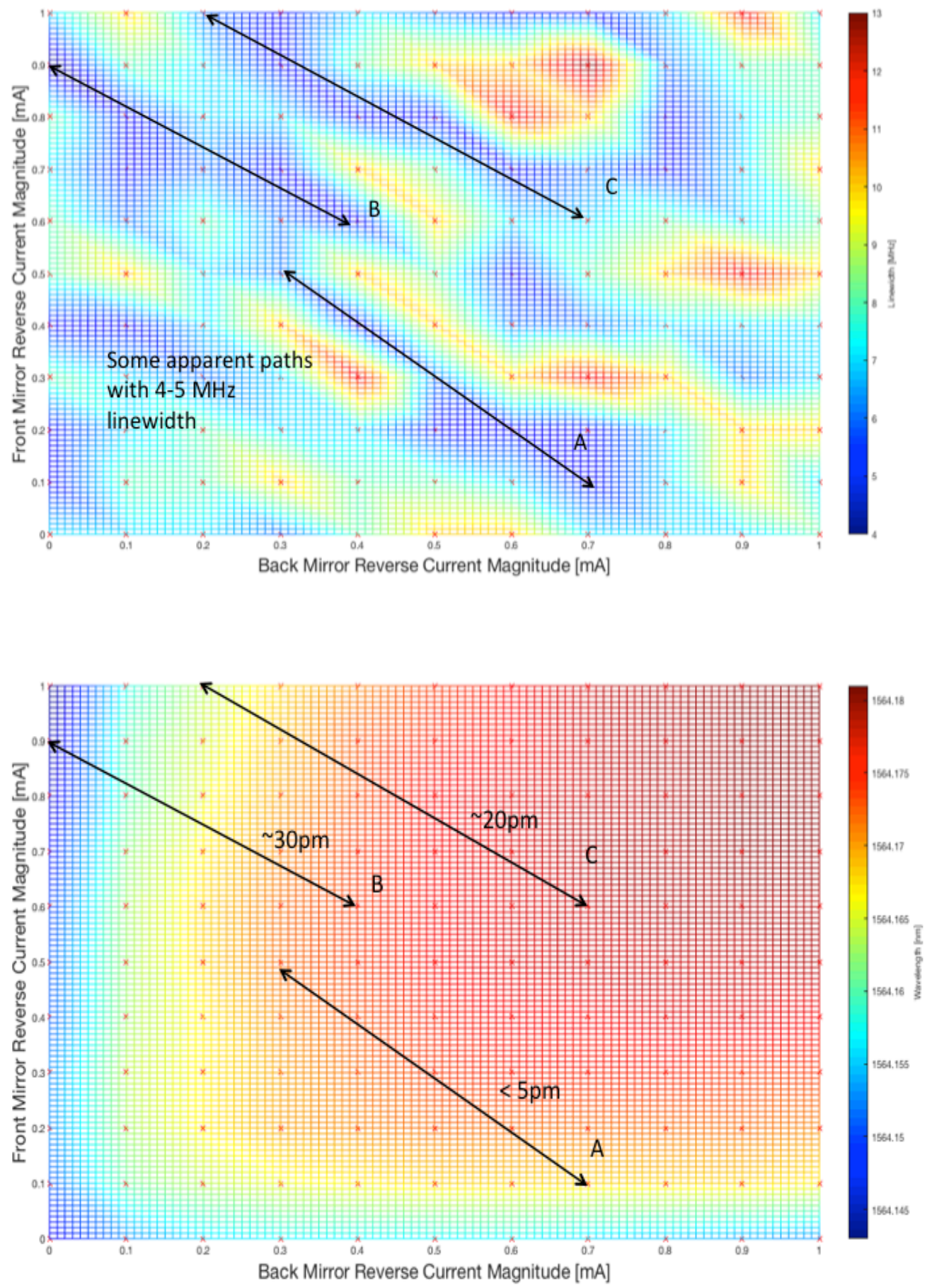

Figure 6.14: Top plow shows potential tuning paths with 4-5MHz linewidth, while the bottom plot shows the wavelength tuning that would accompany these tuning paths. Some narrow linewidth tuning paths have a larger range of wavelengths than others. 
When the phase section is similarly reverse biased in the photo-detector mode, very interesting things happen. For a phase current of $-1 \mathrm{~mA}$, the wavelength tuning range increases by about $25 \%$ from about 40pm in Figure 6.12 to about 50pm, shown in Figure 6.15. Additionally, the linewidth was seen to drop to its intrinsic narrow value between 2 and $4 \mathrm{MHz}$ over the entire map! This effect can be seen in

Figure 6.16. The characteristically low linewidth means that this bias condition could be very promising for LIDAR sweeps. A trace of the self-homodyne signal showing the intrinsically narrow lineshape produced by this bias condition is shown in Figure 6.17
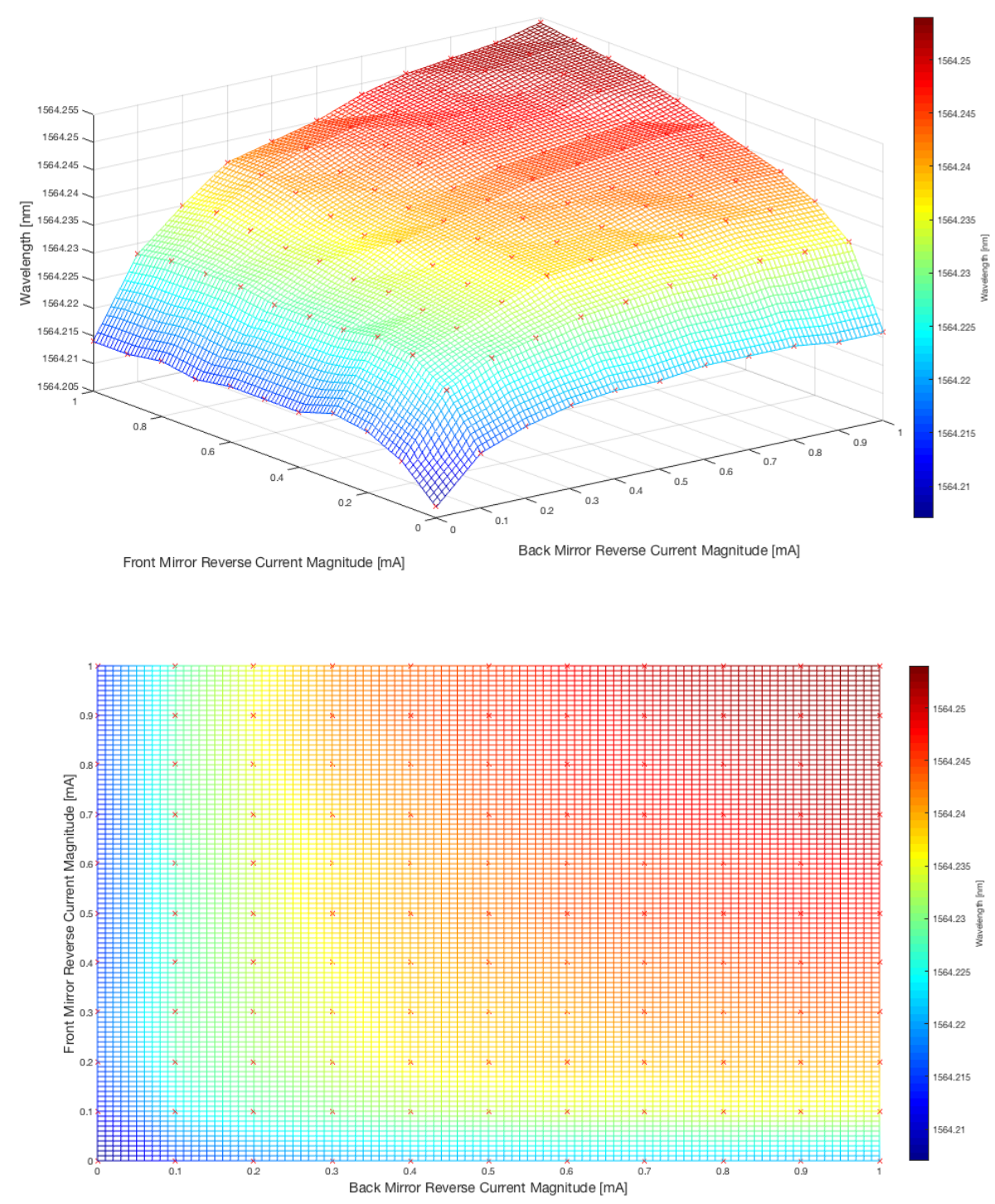

Figure 6.15: Reverse current wavelength map. Phase current is $-1 \mathrm{~mA}$. The addition of reverse phase current both shifts the entire range of wavelengths to the blue, as well as increase the maximum tuning range given by the front and back mirrors alone from 40 picometers to 50 picometers. 


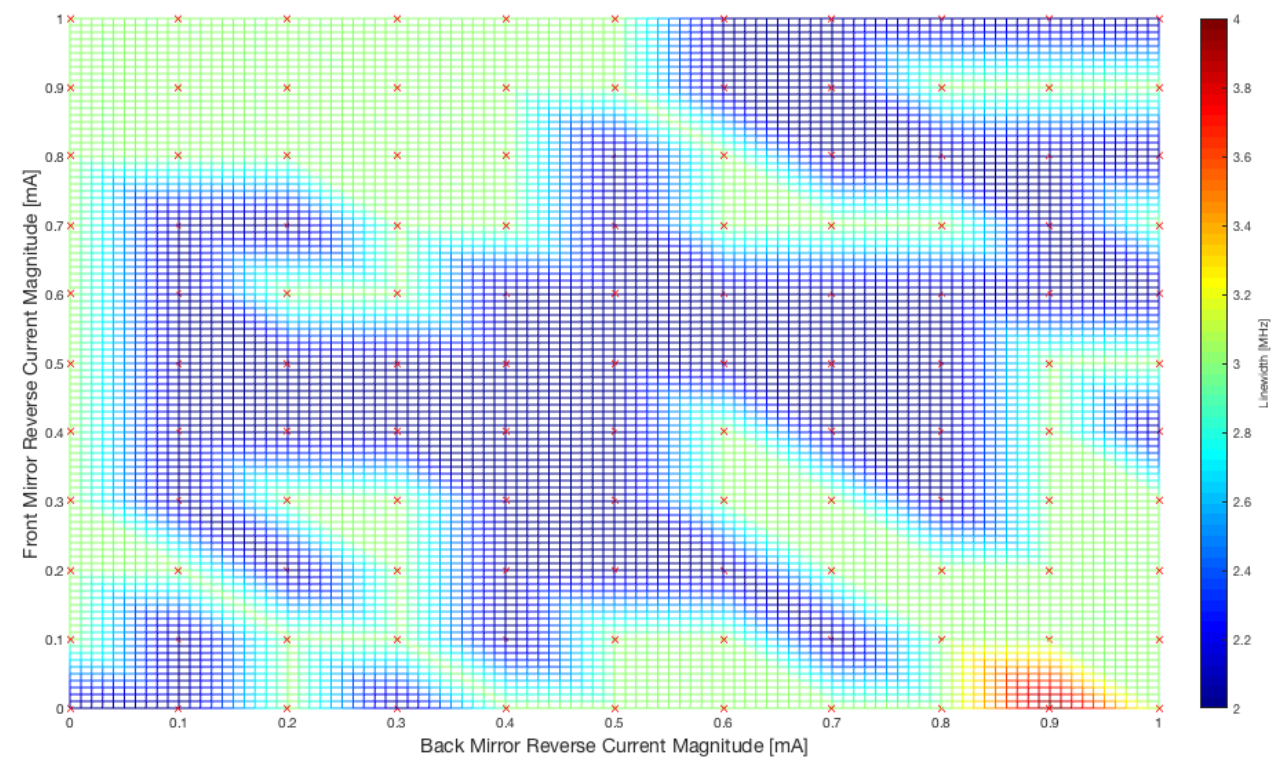

Figure 6.16: Reverse current linewidth map. Phase current is $-1 \mathrm{~mA}$. This plot gives the surprising result that reverse biasing all three sections can lead to linewidth less than $5 \mathrm{MHz}$ over the entire map.

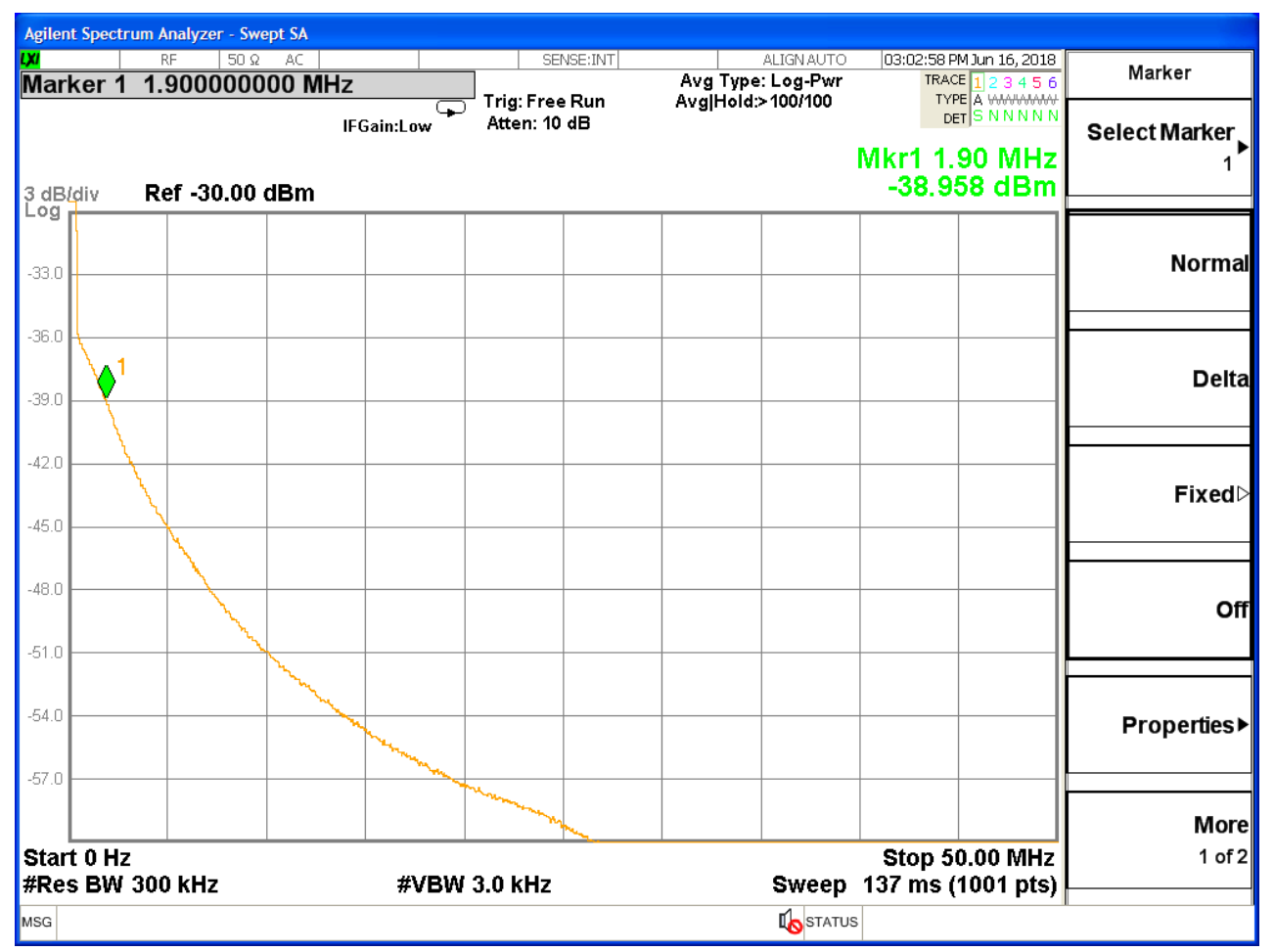

Figure 6.17: Characteristic lineshape for reverse currents into mirror sections and -1mA phase current. Linewidth is seen to be 1.9MHz. All bias conditions shown in Figure 6.15 lead to a lineshape similar to this one. 


\subsection{Resistance Tuning}

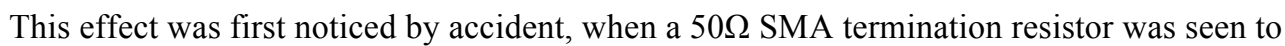
change the wavelength and reduce linewidth when attached to a passive VT-DBR section. Because the linewidth was a narrow $2-4 \mathrm{MHz}$, the idea was explored further and found to provide a tuning range comparable to reverse biasing with an external source. The linewidth was consistently narrow if the diode voltage across the section was not being monitored, but measurement of this voltage with a multi-meter caused an increase in linewidth that was dependent on the value of resistance across the section. The linewidth saw no increase for a short $(\mathrm{R}=0 \Omega)$ and saw an increase up to $20+\mathrm{MHz}$ for $\mathrm{R}=10 \mathrm{k} \Omega$. The resistance tuning was accomplished using the circuits shown in Figure 5.10. The mechanism that causes tuning can be seen in Figure 6.18.

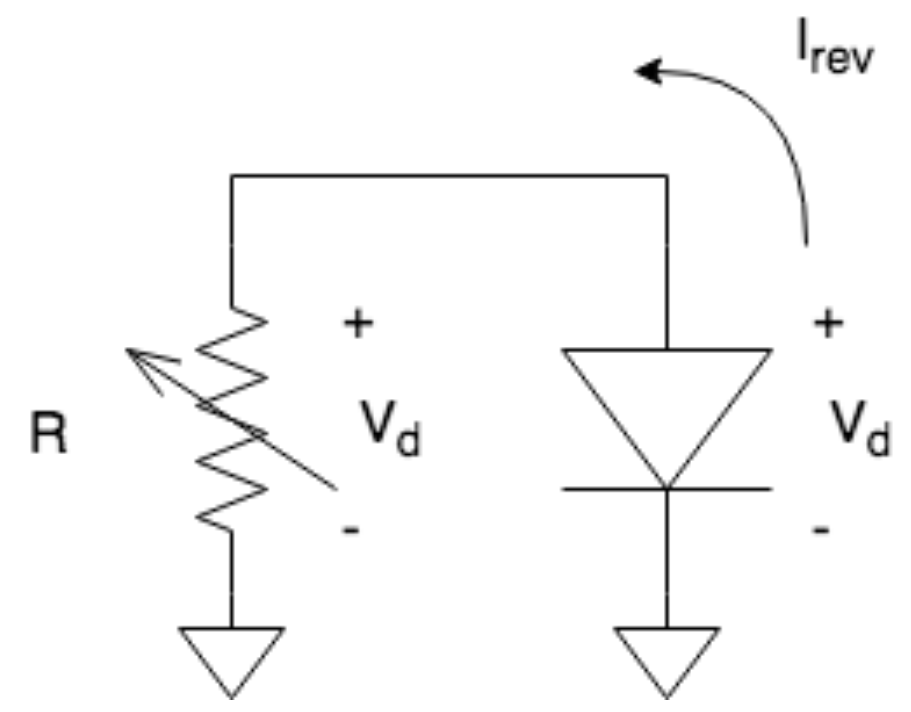

Figure 6.18: Resistance tuning mechanism uses the voltage that occurs across each passive section as a source of bias with a variable resistor to adjust the current.

As the resistance is increased, the laser section diode becomes more forward biased and $\mathrm{V}_{\mathrm{d}}$ increases up to a maximum value around $0.6 \mathrm{~V}$ for the mirror sections or $0.4 \mathrm{~V}$ for the phase section. This diode voltage causes a current to flow out of the laser section and through the potentiometer to ground. The value of this current is seen to increase to a maximum value as the diode becomes more forward biased and then decrease as the resistance continues to increase. An example of this effect can be seen in Figure 6.19 for the back mirror section. 

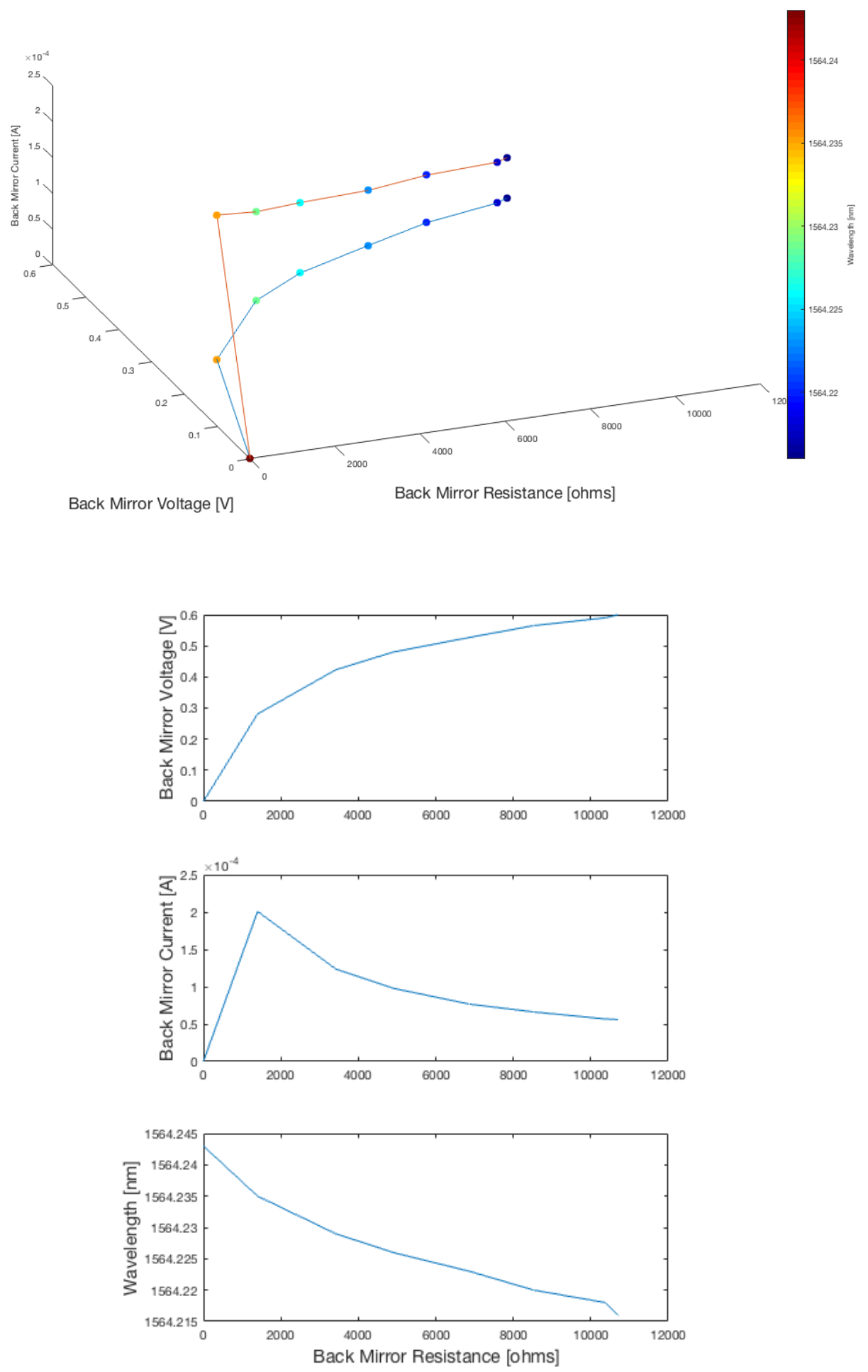

Figure 6.19: Relationship between current, voltage, and wavelength for resistance tuning mechanism. Back mirror section shown. The voltage is seen to increase from zero volts to the expected diode voltage drop of 0.6 volts., while current is seen to decrease with increased resistance. 
Tuning was achieved by varying each section resistance from a short to the maximum value of $10 \mathrm{k} \Omega$. Wavelength maps similar to those for the other tuning mechanisms were also made for the resistance tuning mechanism. Figure 6.20 shows the map for zero resistance across the phase section while Figure 6.21 shows the map for $10 \mathrm{k} \Omega$ across the phase section. The additional resistance across the phase section shifts the entire range about $40 \mathrm{pm}$ to the blue and caused a $50 \%$ increase in the maximum tuning range, from 40pm in Figure 6.20 to 60pm in Figure 6.21.
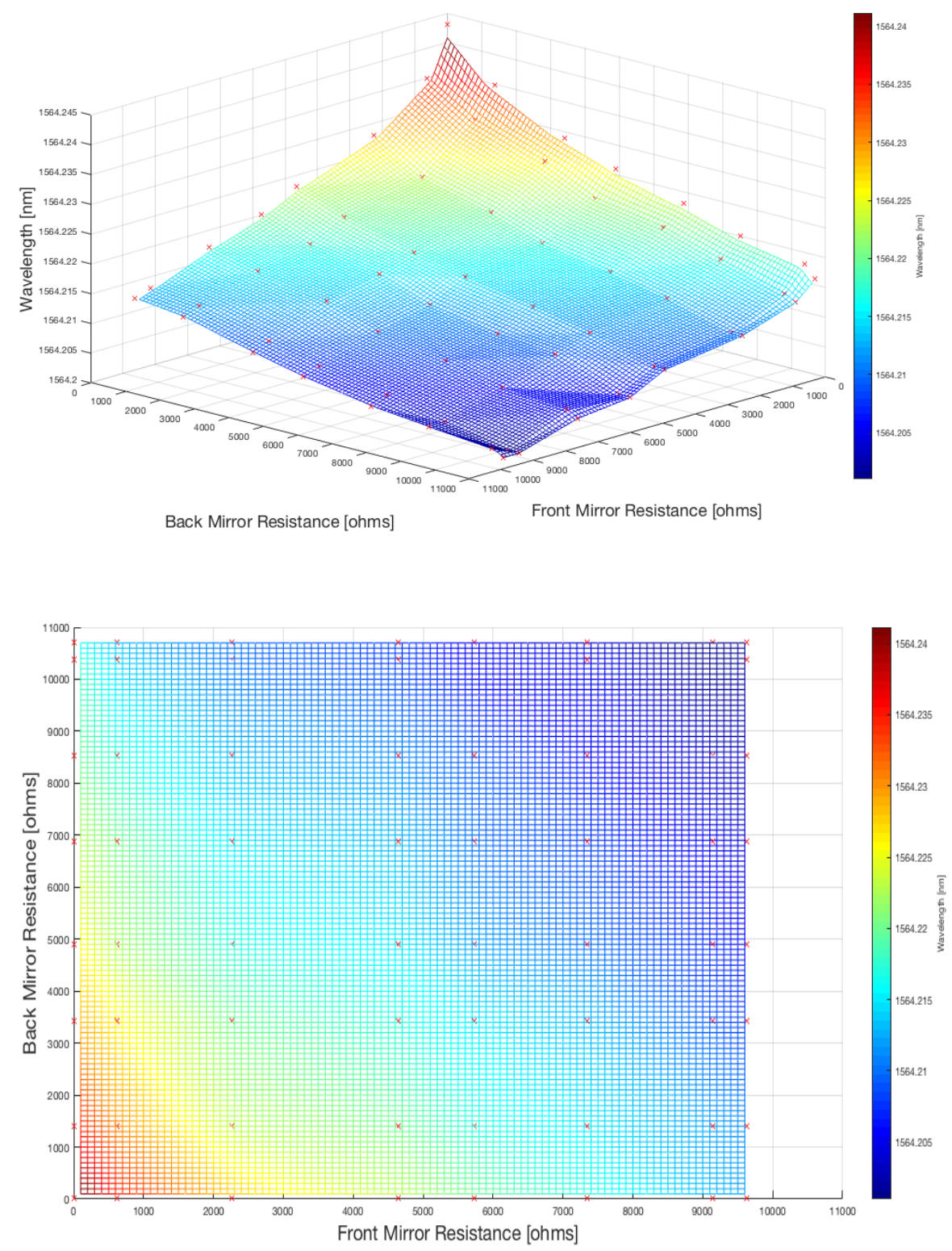

Figure 6.20: Wavelength tuning map for resistance tuning mechanism with $R_{\text {phase }}=0 \Omega$. Resistance tuning is seen to exhibit a smooth tuning surface with a tuning range of $\mathbf{4 0}$ picometers in this case 


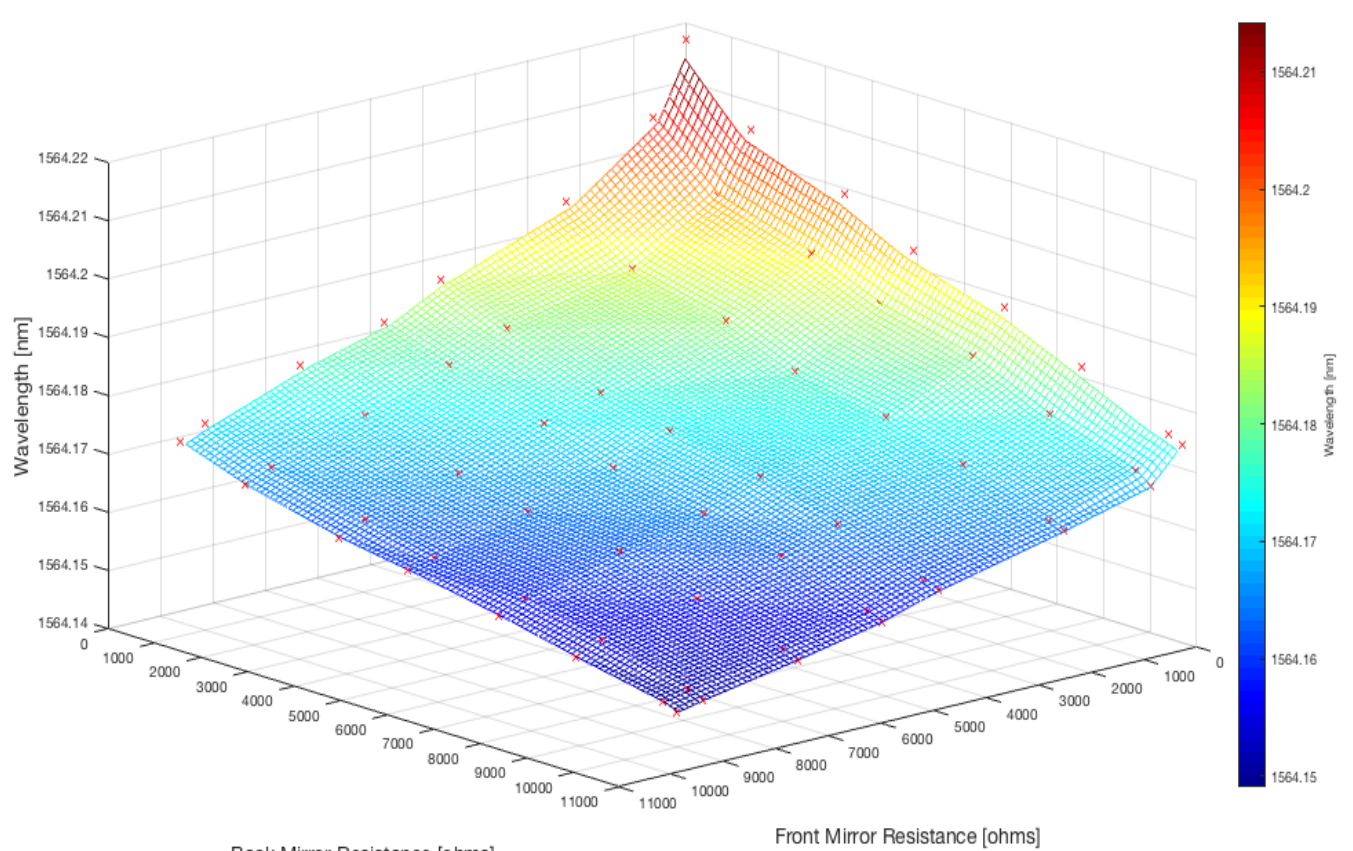

Back Mirror Resistance [ohms]

Front Mirror Resistance [ohms]

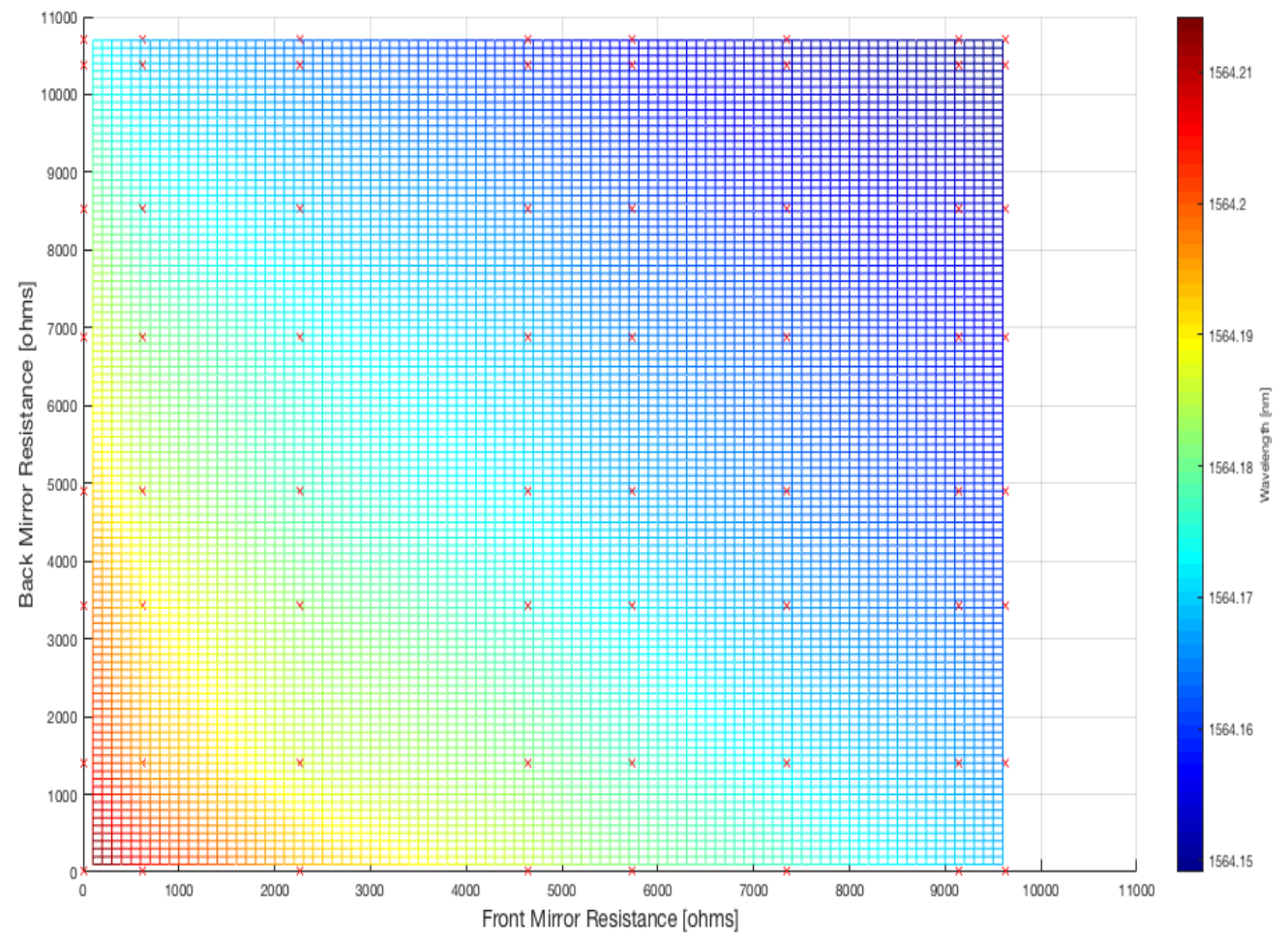

Figure 6.21: Wavelength tuning map for resistance tuning mechanism with $R_{\text {phase }}=10 \mathrm{k} \Omega$. The addition of resistance across the phase section shifts the wavelength to the blue and increases the tuning range from 40 picometers to 60 picometers. 
Since the laser linewidth is an intrinsically narrow value over the entire tuning range when using resistance tuning, this method is an excellent candidate for FMCW LIDAR sweeps. The narrow linewidth characteristic of this tuning method is shown in Figure 6.22.

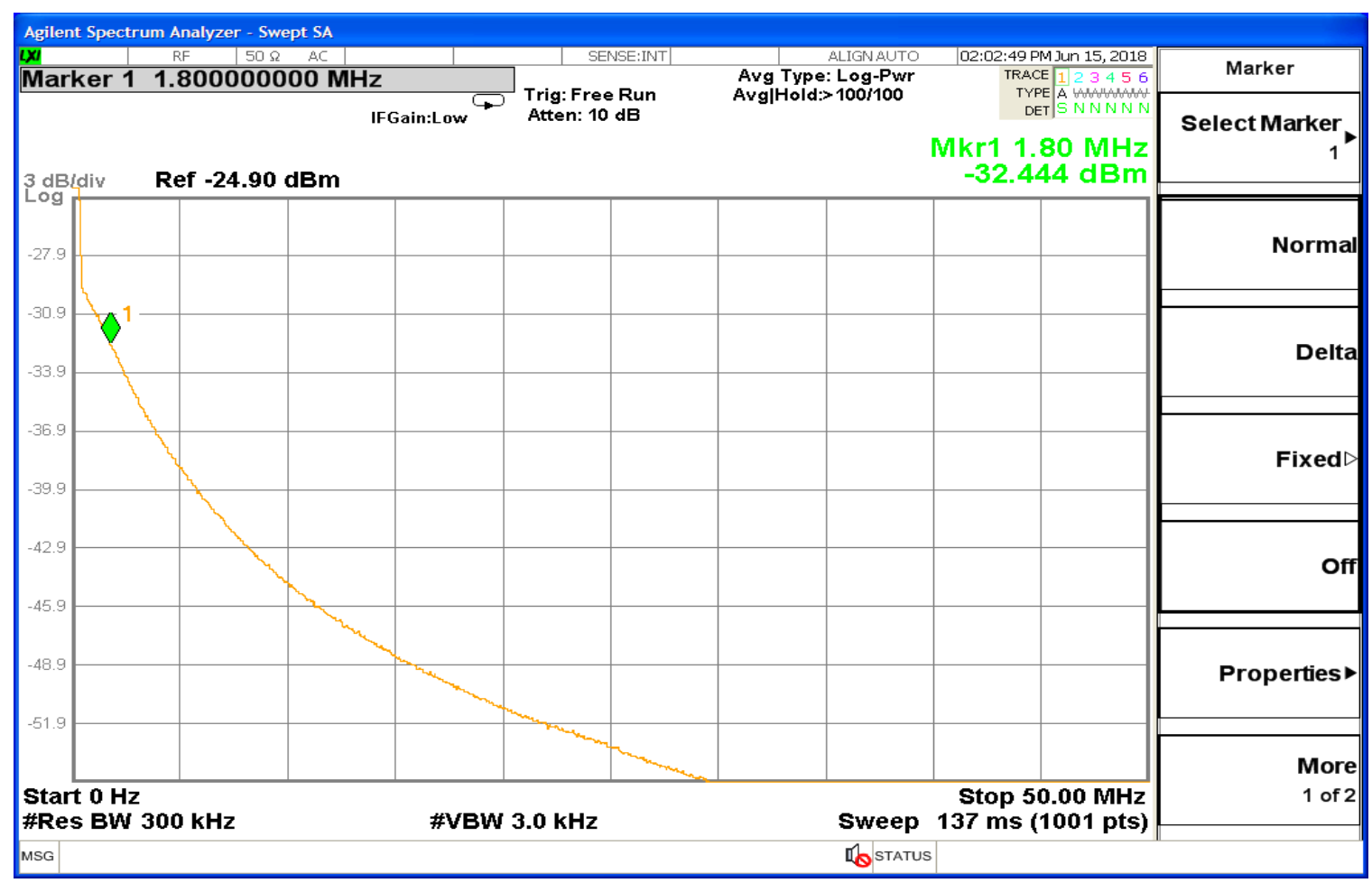

Figure 6.22: Characteristic resistance tuning Self-Homodyne signal with linewidth around 1.8MHz. All bias conditions shown in Figures 6.20 and 6.21 lead to a lineshape similar to this one.

The same tuning can theoretically be achieved using a voltage-controlled resistor (VCR) made from a transistor biased in the ohmic region. A VCR prototype for this application was made using the MPF102 JFET. The MPF102 is an N-channel depletion mode JFET, which means that the device acts like an open switch until a sufficient reverse voltage is applied at the JFET gate, closing the switch. The VCR tuning mechanism is shown in Figure 6.23. 


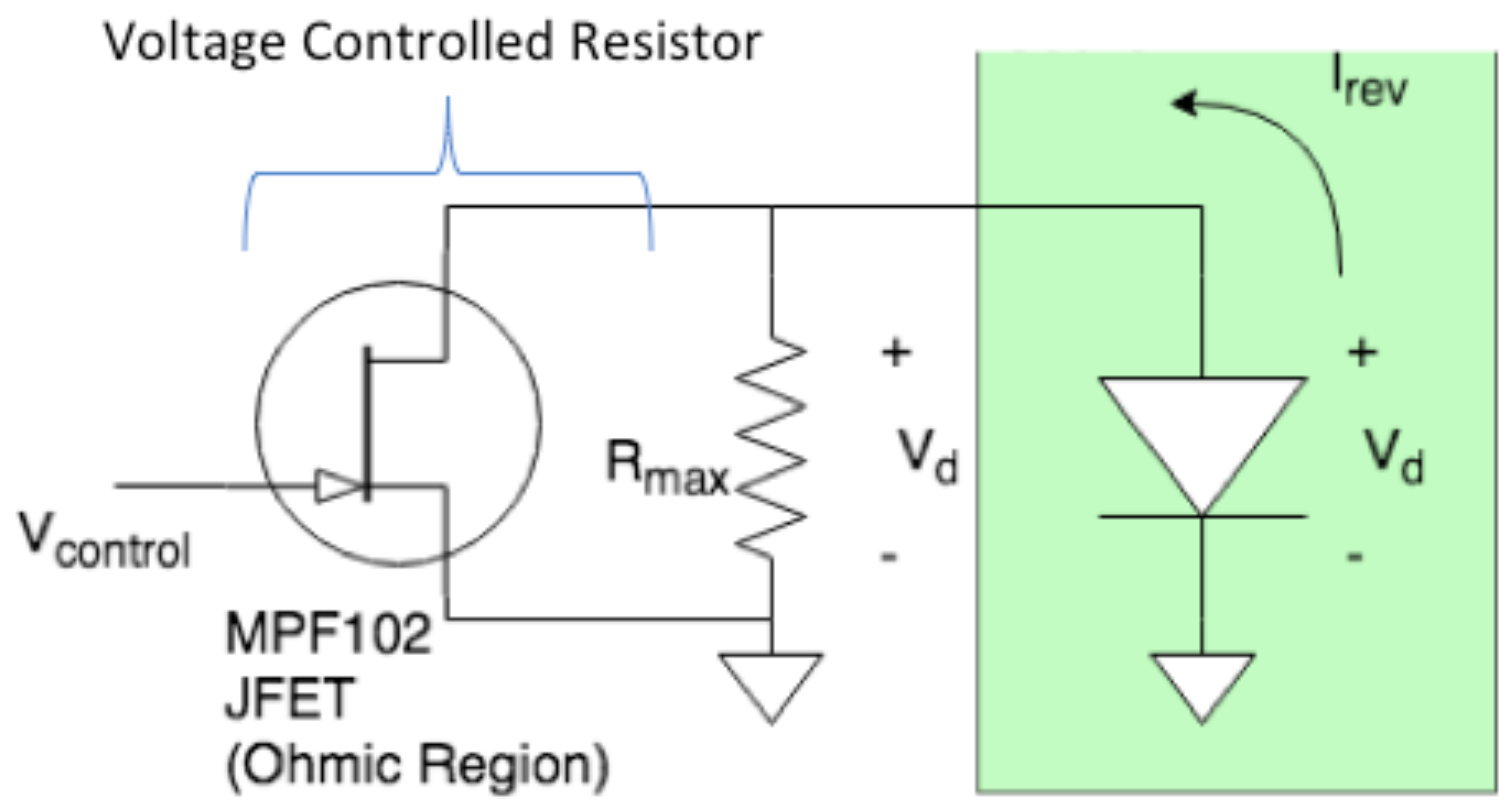

Figure 6.23: Resistance tuning mechanism using a voltage controlled resistor comprised of a JFET in the ohmic region of operation.

The ohmic region of a transistor is defined as the region of the I-V characteristic with a nearly linear relationship between applied voltage and current flow, about $0<\mathrm{V}_{\mathrm{DS}}<3 \mathrm{~V}$. In this region, the transistor behaves like a resistor whose resistance is controlled by the voltage at the transistor gate. Beyond the ohmic region is the saturation region, where the current approaches a saturated value. By staying within the limits of the ohmic region, this device behaves well as a VCR. Figure 6.24 shows a simulated $\mathrm{I}-\mathrm{V}$ characteristic for the device with $-3 \mathrm{~V}<\mathrm{V}_{\mathrm{GS}}<0 \mathrm{~V}$, and Figure 6.25 shows the corresponding measurement using a Semiconductor Parameter Analyzer. 


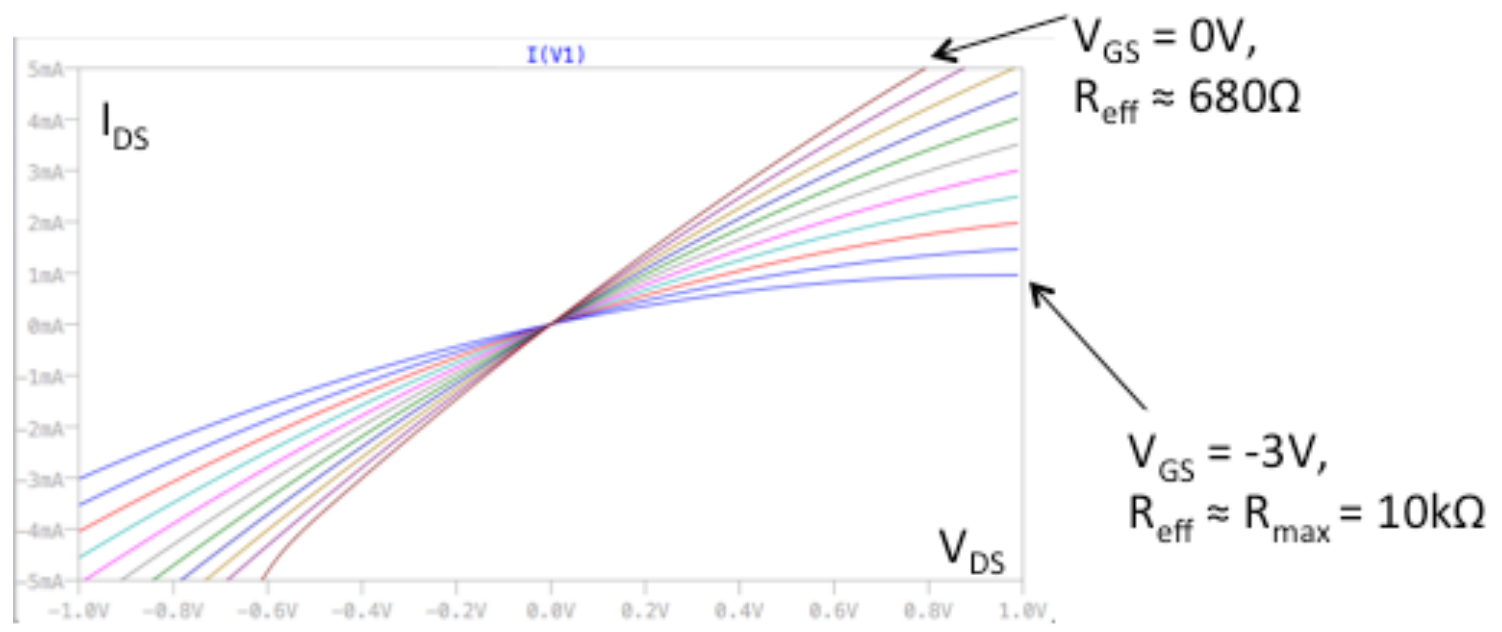

Figure 6.24: Simulated I-V characteristics for MPF102 JFET VCR with $R_{\max }=10 \mathrm{k} \Omega$. This simulation shows that the voltage controlled resistor can take on any resistance between about $700 \Omega$ and $10 \mathrm{k} \Omega$.

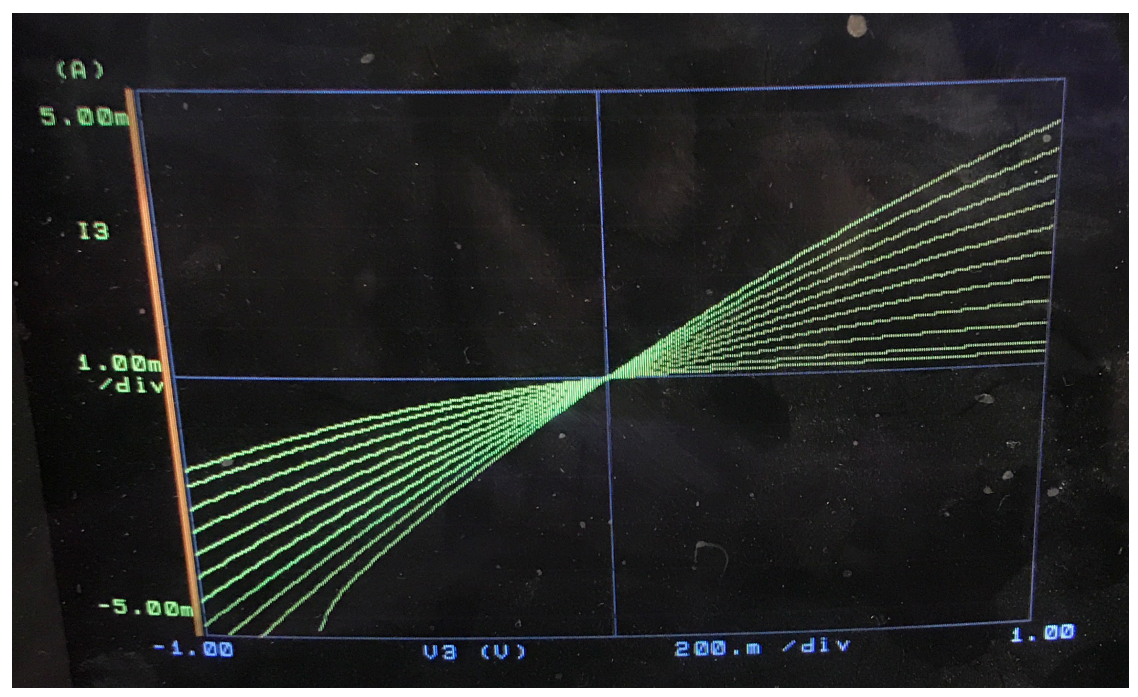

Figure 6.25: Measured I-V characteristics for MPF102 JFET VCR with $R_{\max }=10 \mathrm{k} \Omega$ using a semiconductor parameter analyzer. Measurement matches with simulation.

\subsection{Frequency Response Comparison of Forward Biasing and Resistance Tuning}

It is clear that the resistance tuning mechanism can be used to tune the laser wavelength with narrow linewidth, but how fast can the laser respond to changes in resistance? To attempt to answer this question, the frequency response of both the JFET tuning mechanism and the direct forward current injection tuning mechanism were measured using a vector network analyzer (VNA). The VNA measurement diagram is shown in Figure 6.26. The constants $\mathrm{K}_{\text {disc }}$ and $\mathrm{K}_{\text {laser }}$ are yet undetermined, and needed for simulation of the frequency response of each tuning mechanism. $\mathrm{K}_{\text {laser }}$ is the amount of 
frequency change on the laser output for a given change in phase section current, while $\mathrm{K}_{\text {disc }}$ is the amount of photocurrent in $\mathrm{mA}$ that arises per $\mathrm{GHz}$ of frequency modulation on the signal into the frequency discriminator.

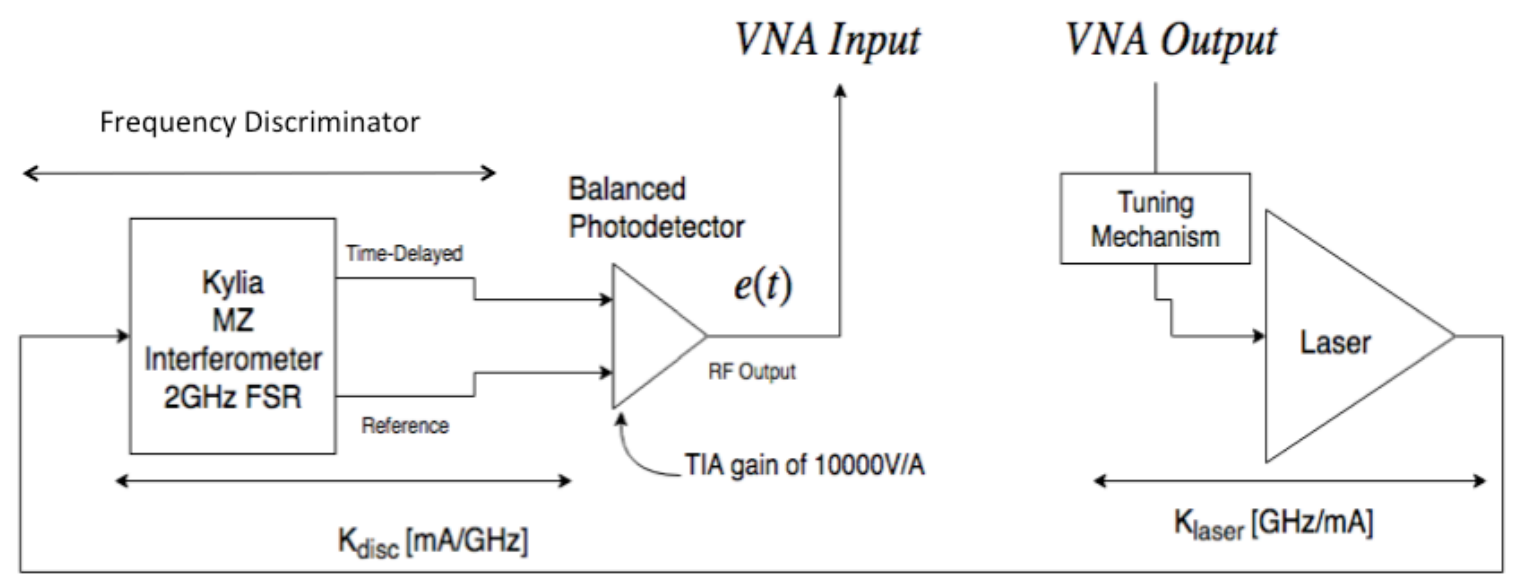

Figure 6.26: VNA measurement diagram. Need to determine $K_{\text {disc }}$ and $K_{\text {laser }}$ for the forward current injection and reverse bias tuning mechanisms.

To measure $\mathrm{K}_{\text {disc }}$, the photocurrent variation vs. frequency was plotted at the output of the balanced photo-detector. This measurement, shown in Figure 6.27, provided an estimate of the maximum photocurrent variation as well as the free spectral range (FSR) of the interferometer for a given optical power into the filter. The constant $\mathrm{K}_{\text {disc }}$ is defined as the maximum slope of this plot, or the slope at the quadrature position. The value of $\mathrm{K}_{\text {disc }}$ can be estimated as $\frac{\max (i)}{F S R / 2}=\frac{2 \max (i)}{F S R}=0.084 \mathrm{~mA} / \mathrm{GHz}$

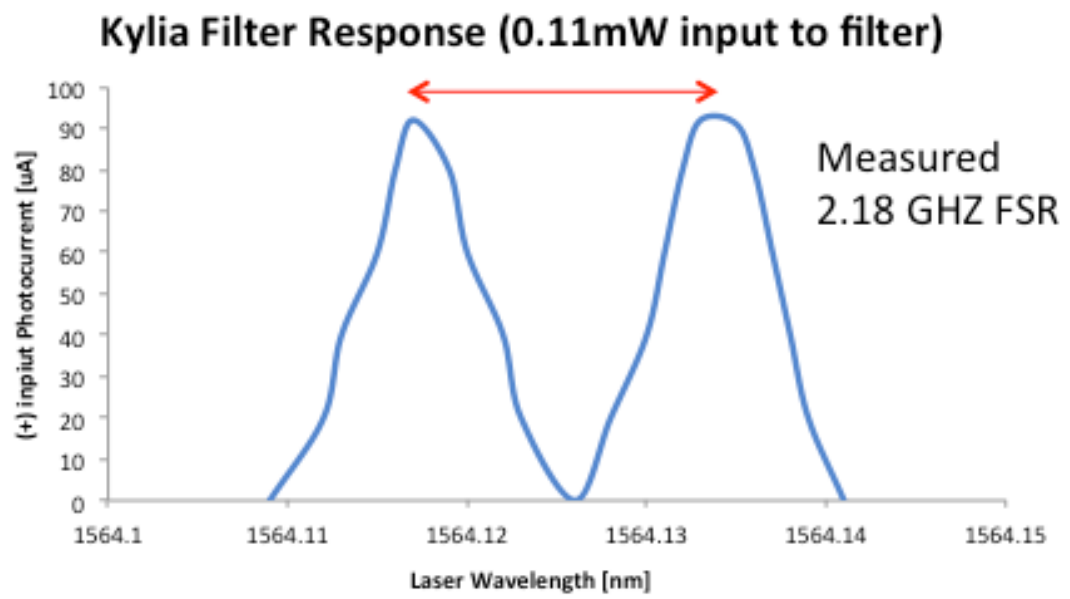

Figure 6.27: Determination of $\mathrm{K}_{\mathrm{disc}}$, which is the maximum slope of the plot of detected photocurrent vs. frequency for a given optical power level. 
To measure $\mathrm{K}_{\text {laser, }}$ a plot of laser wavelength vs. phase current was made for both forward and reverse current tuning. Since these curves are somewhat linear, the slope of a linear regression line is a

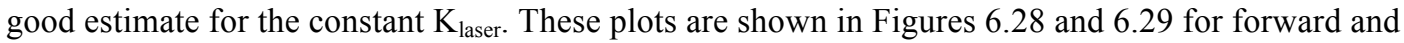
reverse currents respectively. In Figure 6.28 , the slope is $-0.0007 \mathrm{~nm} / \mu \mathrm{A}$, or equivalently $-85 \mathrm{GHz} / \mathrm{mA}$ at $1564 \mathrm{~nm}$ for small forward currents. In Figure 6.29 , the slope is $0.00009 \mathrm{~nm} / \mu \mathrm{A}$, or equivalently 11 $\mathrm{GHz} / \mathrm{mA}$ at $1564 \mathrm{~nm}$ for small reverse currents. The laser wavelength is always tuned towards the blue for an increase in current magnitude, regardless of the direction of current flow.

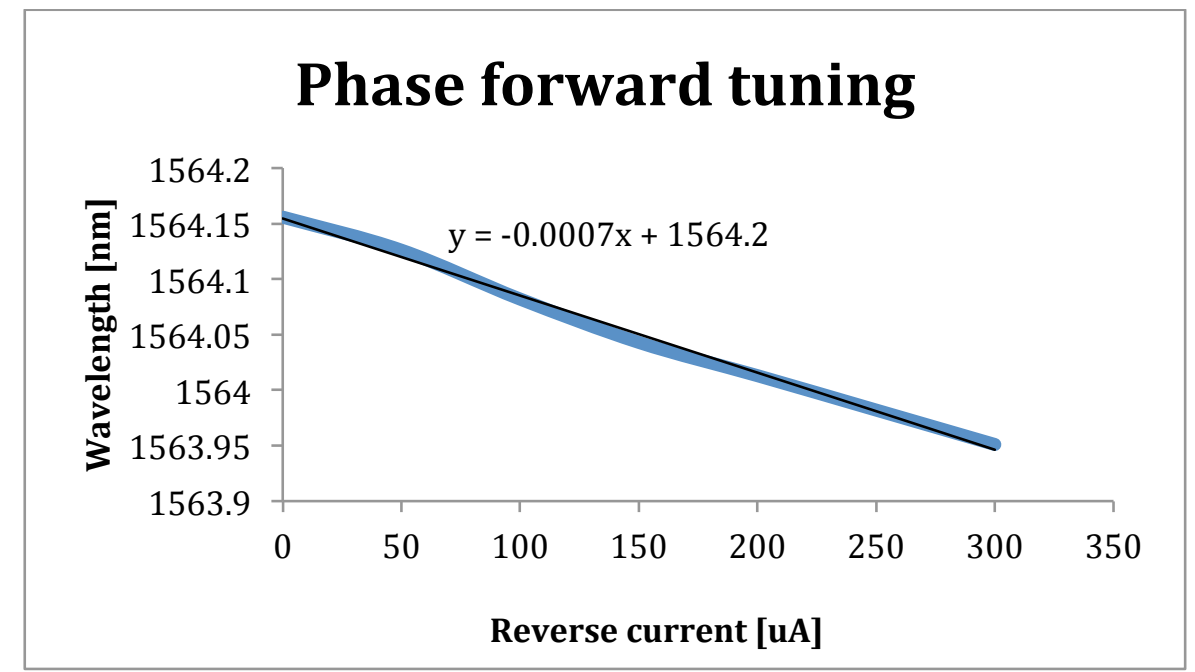

Figure 6.28: Determination of $K_{\text {laser }}$ for forward current injection. It is found that $K_{\text {laser }}=\mathbf{- 8 5 G H z} / \mathbf{m A}$ for forward current injection.

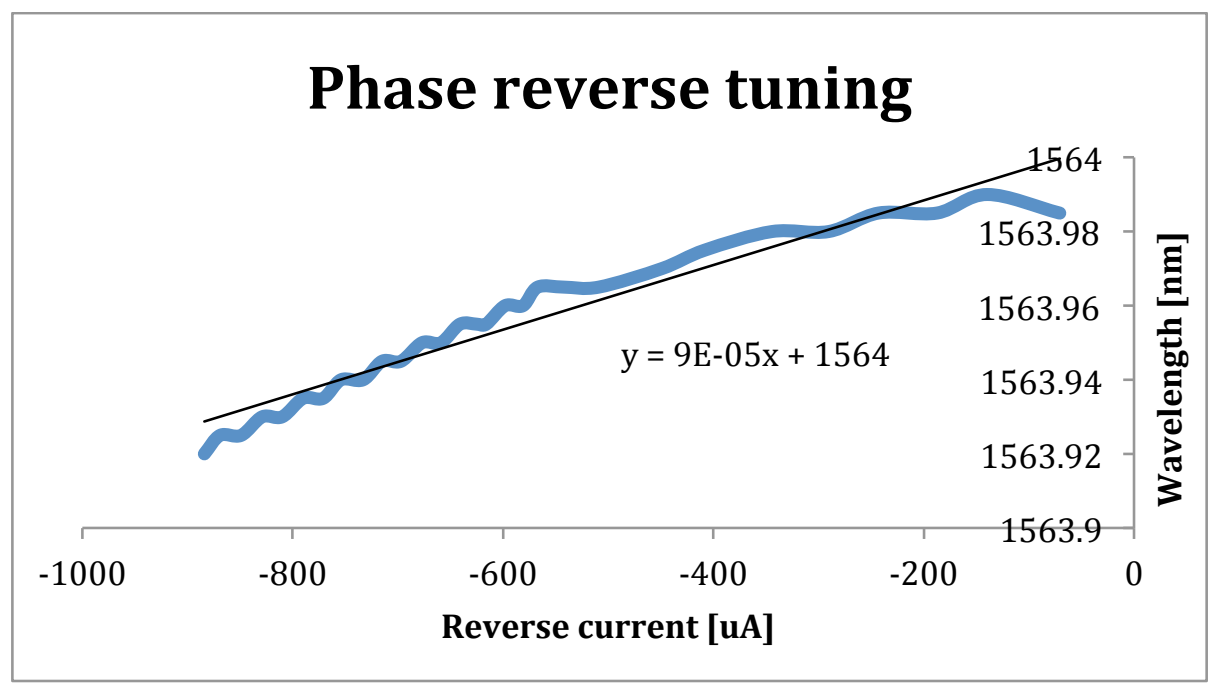

Figure 6.29: Determination of $K_{\text {laser }}$ for reverse current reverse biasing. It is found that $\mathrm{K}_{\text {laser }}=11 \mathrm{GHz} / \mathrm{mA}$ for reverse biasing. 
Now that the values of $\mathrm{K}_{\text {disc }}$ and $\mathrm{K}_{\text {laser }}$ are known, it is possible to simulate the frequency response of both tuning mechanisms. The simulation was done in LTspice, and the schematic for both simulations are shown in Figure 6.30. The phase section was modeled as a $0.6 \mathrm{~V}$ voltage source and the balanced photodetector was modeled as a pair of behavioral current sources with equal and opposite current magnitudes set by $\mathrm{K}_{\text {disc }} * \mathrm{~K}_{\text {laser }}{ }^{*} \mathrm{I}_{\text {phase }}$ feeding into an ideal trans-impedance amplifier with a gain of $10000 \mathrm{~V} / \mathrm{A}$.
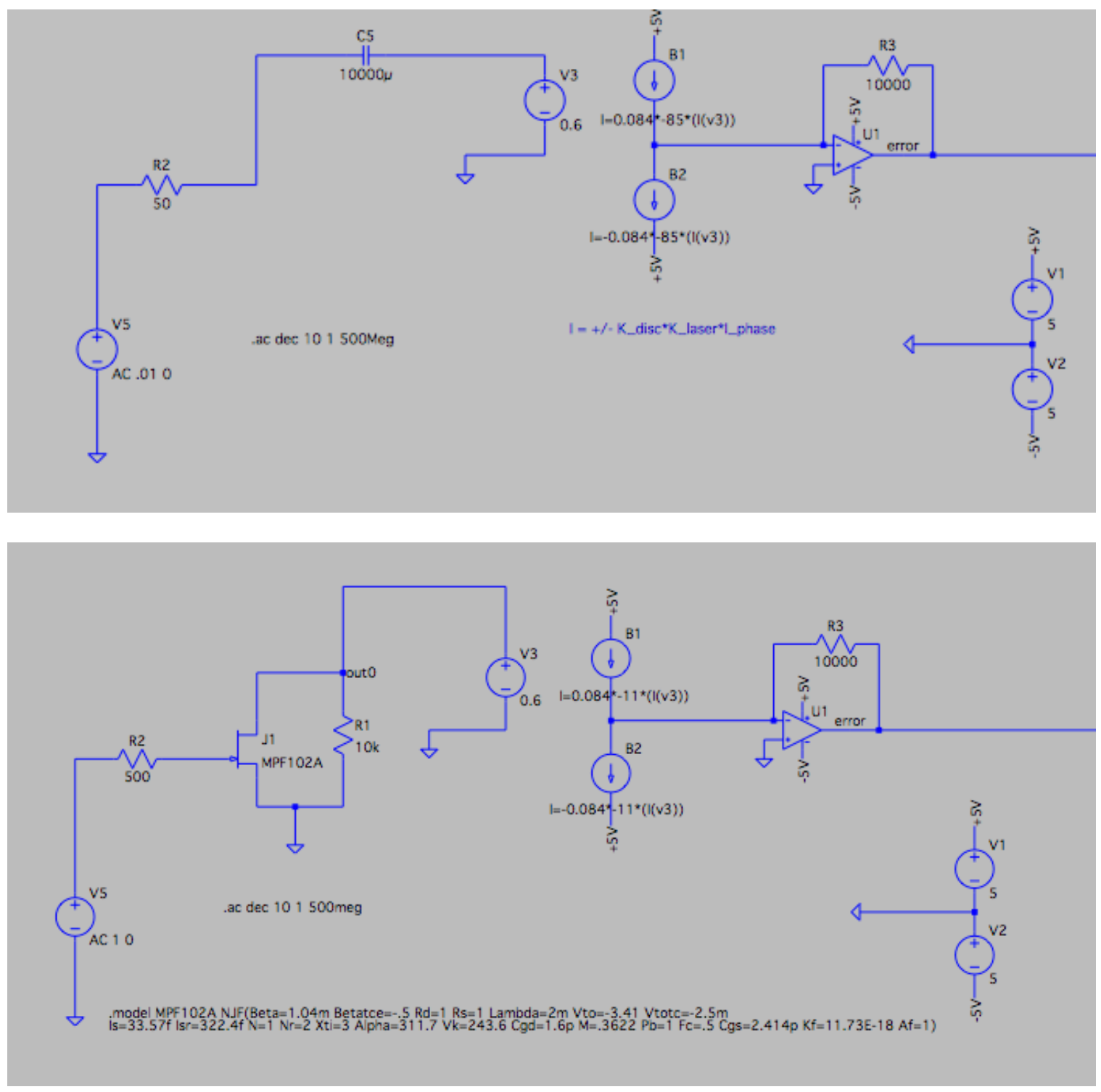

Figure 6.30: LTspice simulation schematic for direct injection (top) and JFET tuning (bottom). In both cases, the laser phase section is modeled as a voltage source and the balanced photo-detector is modeled as two dependent current sources with equal and opposite currents given by

$$
\mathbf{K}_{\text {dise }} * \mathbf{K}_{\text {laser }} * \mathbf{I}_{\text {phase }}
$$


Figures 6.31 and 6.32 show the results of the simulations shown in Figure 6.30. The simulated direct injection tuning method is seen to have a much larger signal magnitude when compared to the JFET tuning method, and has an odd increase in magnitude at higher frequencies. The JFET tuning method has a simulated $-3 \mathrm{~dB}$ bandwidth over 100MHz.

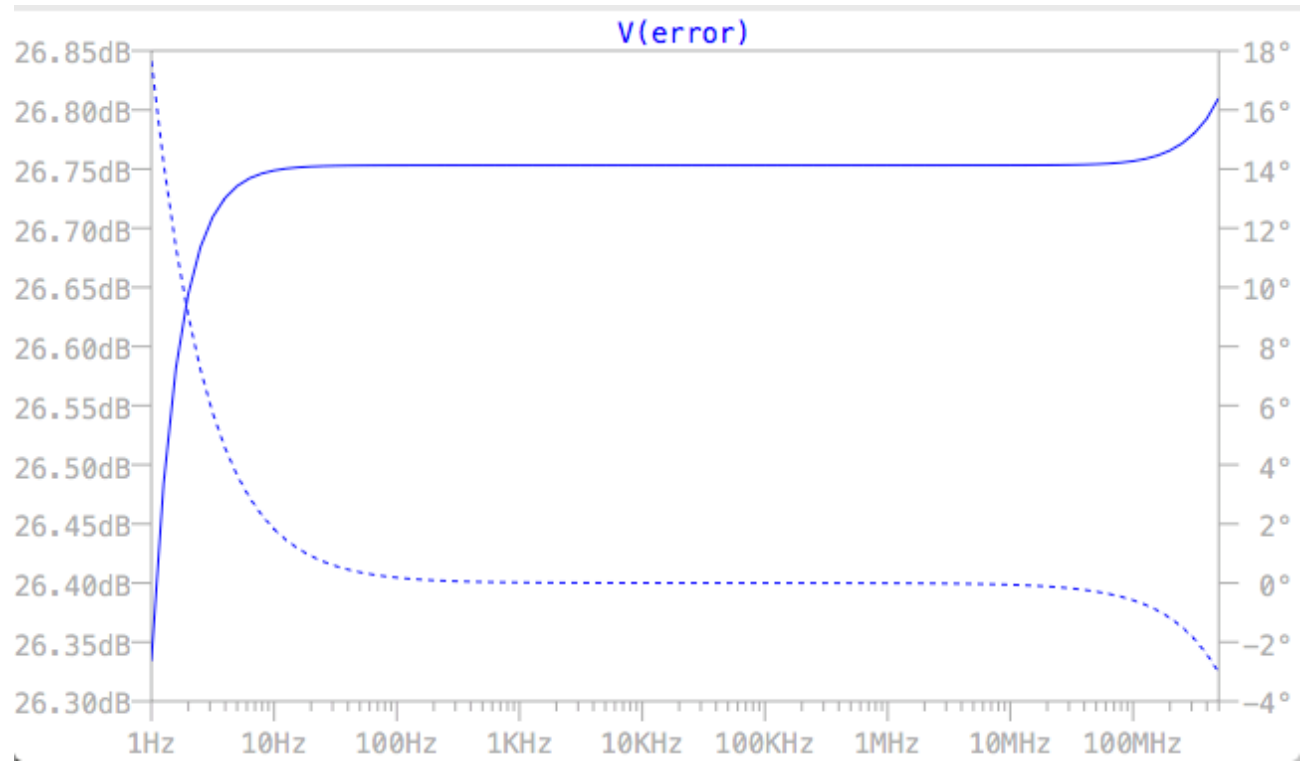

Figure 6.31: Simulated frequency response for direct injection tuning, shown in the top plot of Figure 6.30. The solid line shows the signal magnitude while the dotted line shows that phase shift.

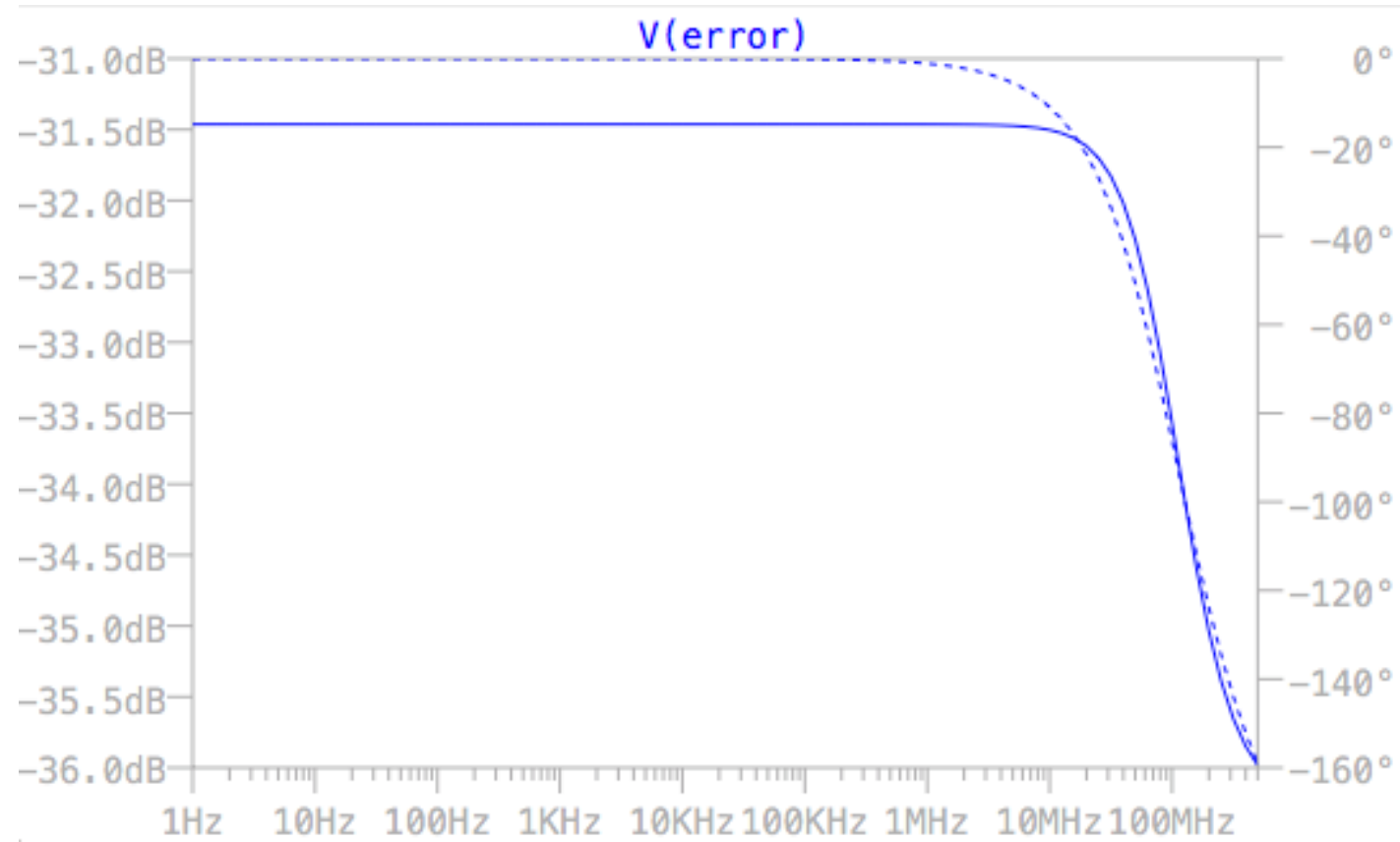

Figure 6.32: Simulated frequency response for JFET resistance tuning, shown in the bottom plot of Figure 6.30. The solid line shows the signal magnitude while the dotted line shows that phase shift. 
In addition to simulation, these measurements were also taken with a $5 \mathrm{~Hz}-500 \mathrm{MHz} \mathrm{VNA}$. The results of these VNA measurements are shown in Figure 6.33 for the direct injection tuning mechanism and Figure 6.34 for the JFET resistance tuning mechanism. As the simulation predicted, the frequency response of the direct injection mechanism is much larger in signal magnitude than that of the JFET tuning mechanism. Both responses start to fall off around $350 \mathrm{MHz}$, which is the actual bandwidth of the balanced photodetector in use. The JFET resistance tuning case shows an increase in signal magnitude with frequency until the photo-detector bandwidth forces the magnitude down again. Additionally, in both cases the signal magnitude dips down as much as $20 \mathrm{~dB}$ at certain frequencies. The rise in signal magnitude for the JFET case and the dips in signal magnitude in either case are not yet fully understood. In a practical LIDAR system, each tuning mechanism needs a sweep repetition rate less than $10 \mathrm{MHz}$. This means that only a portion of the first $50 \mathrm{MHz}$ on these measurements is actually relevant and both tuning mechanisms can be tuned with repetition rates of less than $10 \mathrm{MHz}$ without significant signal degradation.

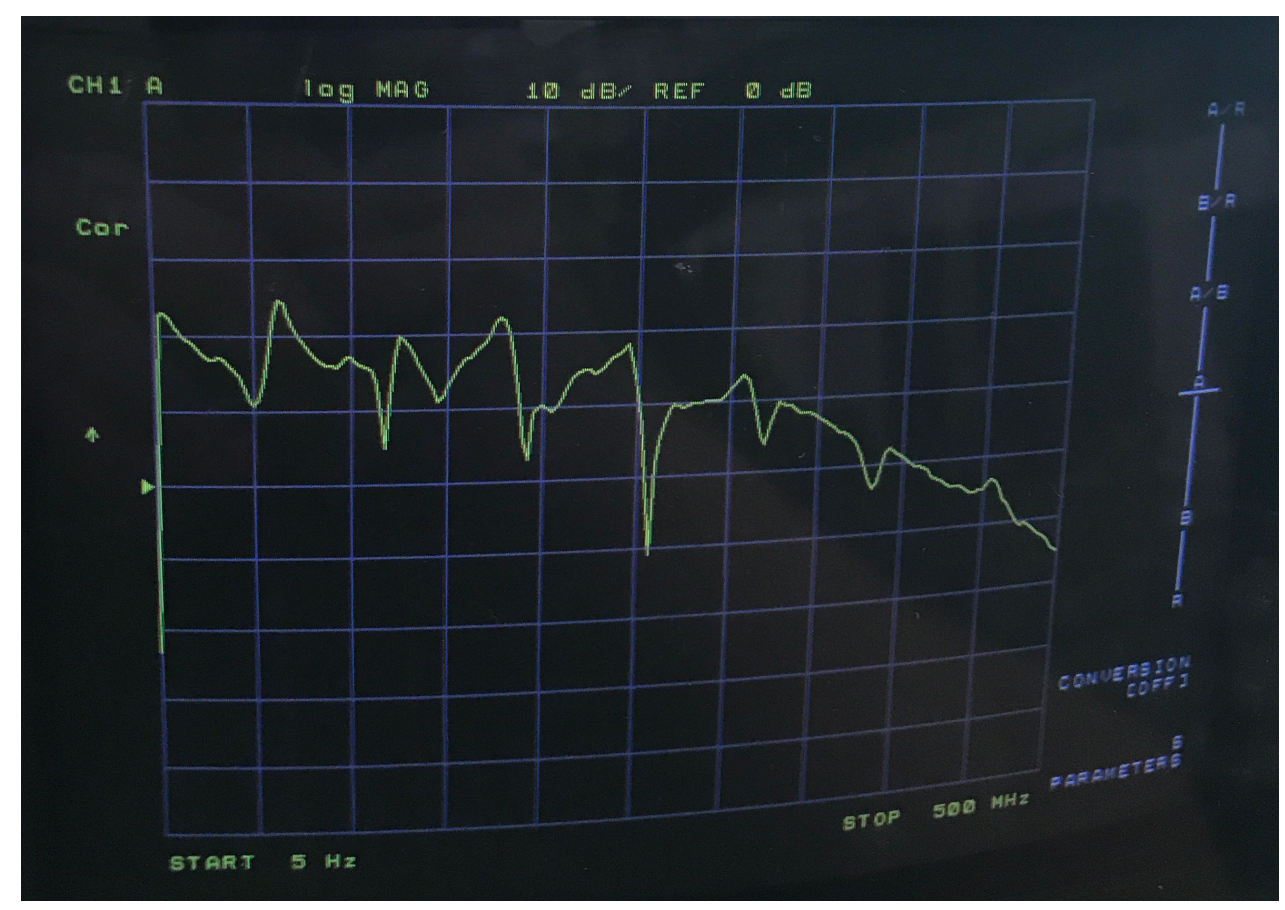

Figure 6.33: Measured frequency response for direct current injection tuning mechanism. DC gain is seen to be above 20dB, which matches the corresponding simulation shown in Figure 6.31. Dips in signal magnitude occur roughly every $50 \mathrm{MHz}$, but this effect is not fully understood. 


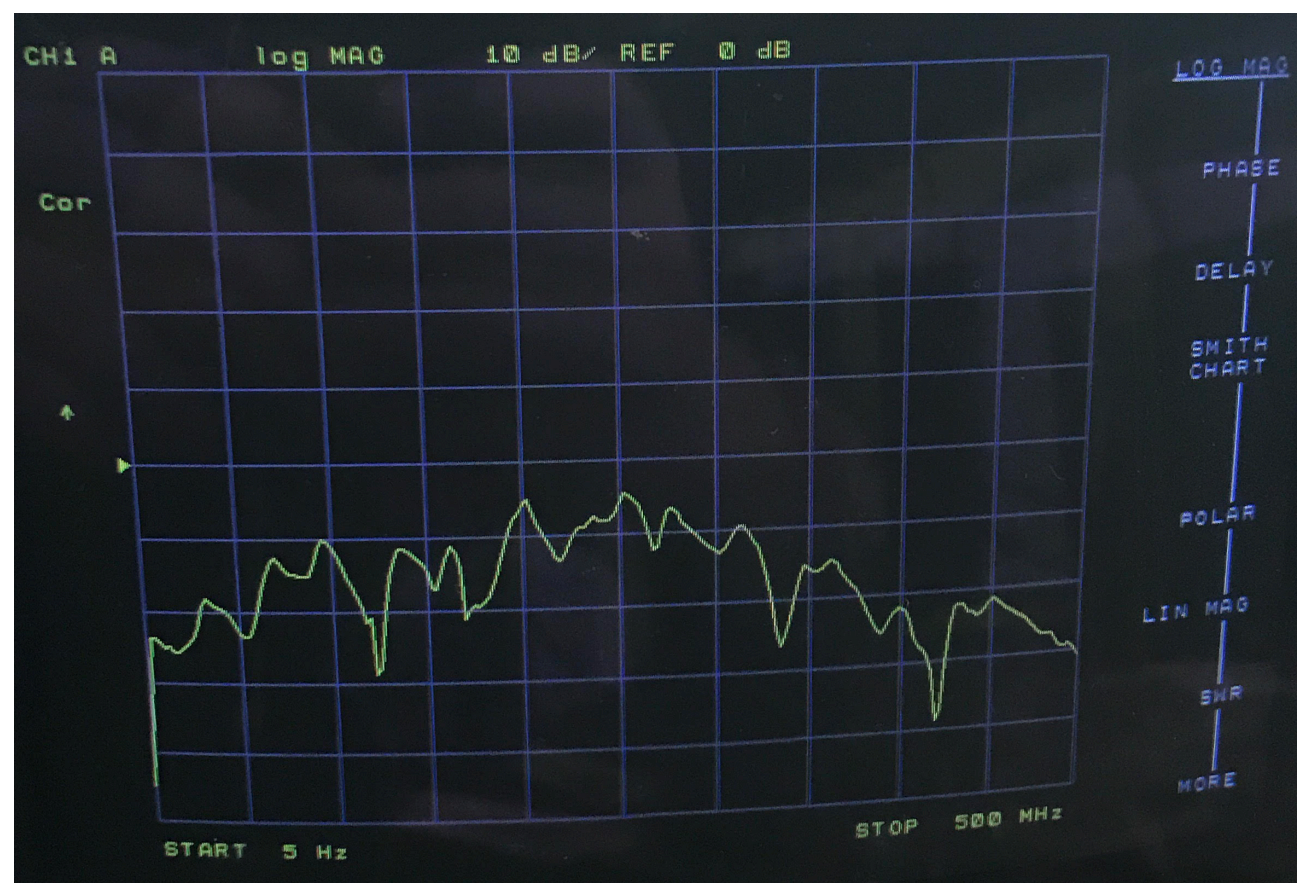

Figure 6.34: Measured frequency response for JFET resistance tuning mechanism. DC gain is seen to be less than -20dB, which matches the corresponding simulation shown in Figure 6.31. The signal magnitude rises more than $10 \mathrm{~dB}$ in the first $250 \mathrm{MHz}$ and dips in signal magnitude occur roughly every $50 \mathrm{MHz}$, but these effects are not fully understood. 


\section{Chapter 7: Reducing Linewidth Using Optoelectronic Feedback}

\subsection{Frequency Locked Loops}

A frequency-locked loop (FLL) is a feedback control system that forces an output oscillator frequency to match that of an input. The basic components of a FLL include a phase comparator, a loop filter, and a voltage-controlled oscillator (VCO). Figure 7.1 shows the basic block diagram of a FLL.

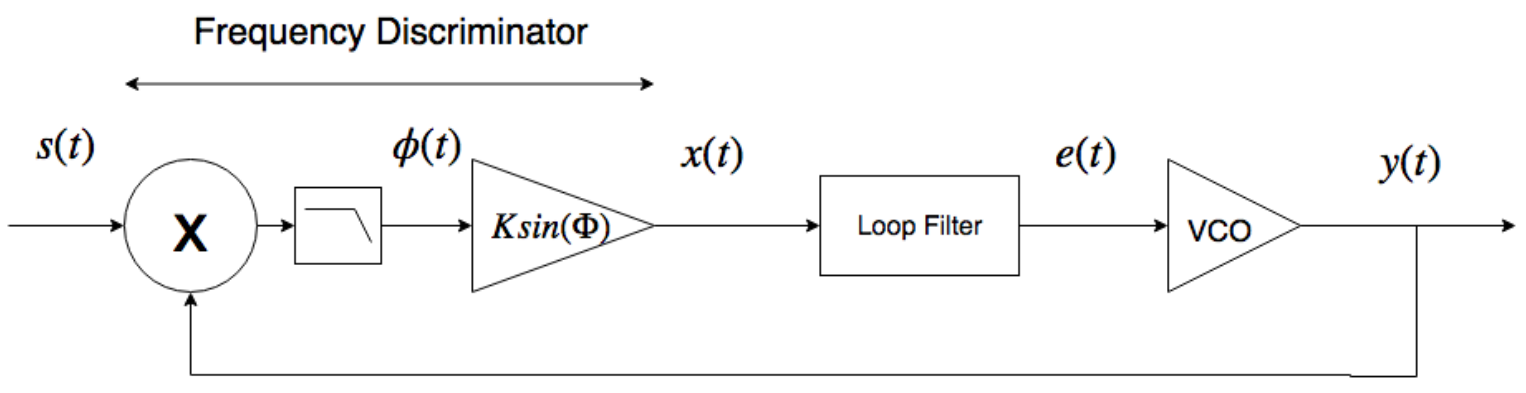

Figure 7.1: Basic block diagram of a FLL showing the main components: a frequency discriminator, a loop filter, and a voltage-controlled oscillator.

The FLL works by comparing the VCO output frequency with the frequency of a reference oscillator $s(t)$.

The signal at $x(t)$ is a sinusoid whose argument contains any phase or frequency difference between the VCO and reference signals. The signal $x(t)$ is sent through a loop filter whose output is the error signal $e(t)$. The error signal is then input into the VCO to close the loop. When this circuit is locked, the output VCO frequency should exactly match that of the reference, while relative phases may have an offset or even drift.

\subsection{Optical FLL to Reduce Laser Phase Noise}

The general FLL design can be adapted for use in optical systems as well. These systems are known as optical frequency-locked loops (OFLL) and are slightly more complex than an electronic FLL. This complexity arises from the need to convert optical signals to electrical signals. A block diagram of a general OFLL is shown in Figure 7.2. Note the similarities between Figures 7.1 and 7.2. 


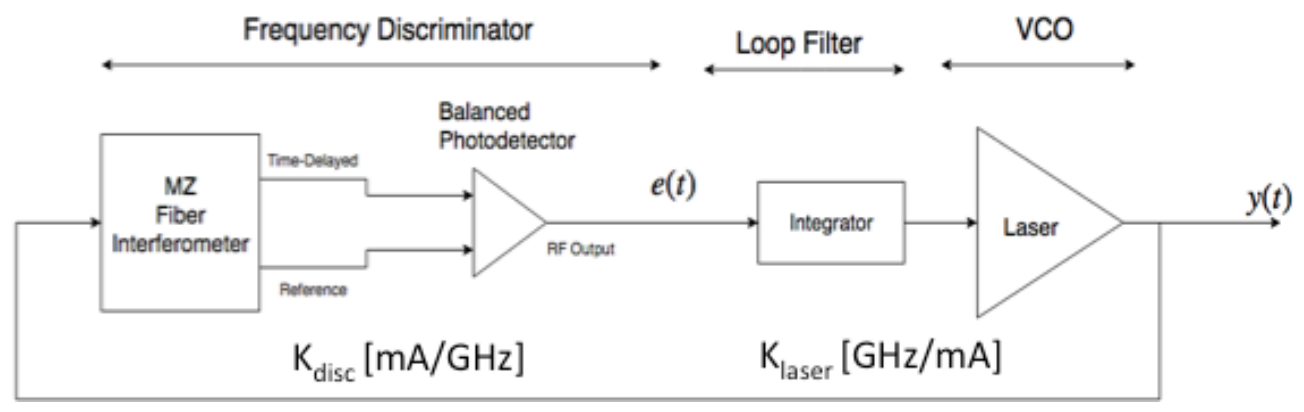

Figure 7.2: Basic block diagram of an OFLL. The frequency discriminator is a fiber optic interferometer and balanced photo-detector, the loop filter is an op-amp integrator, and the VCO is the laser.

The experimental OFLL can be see to be the "feedback section" of Figure 5.11, and a loop block diagram for the system is shown in Figures 7.3 and 7.4. Figure 7.3 shows the OFLL with direct current injection into the phase section as a feedback tuning mechanism, while Figure 7.4 shows OFLL with a voltage-controlled resistance tuning mechanism on the phase section. In both systems, the resistance tuning mechanism discussed in Section 6.4 is used on both mirror sections to adjust the laser frequency to lock while maintaining a narrow linewidth.

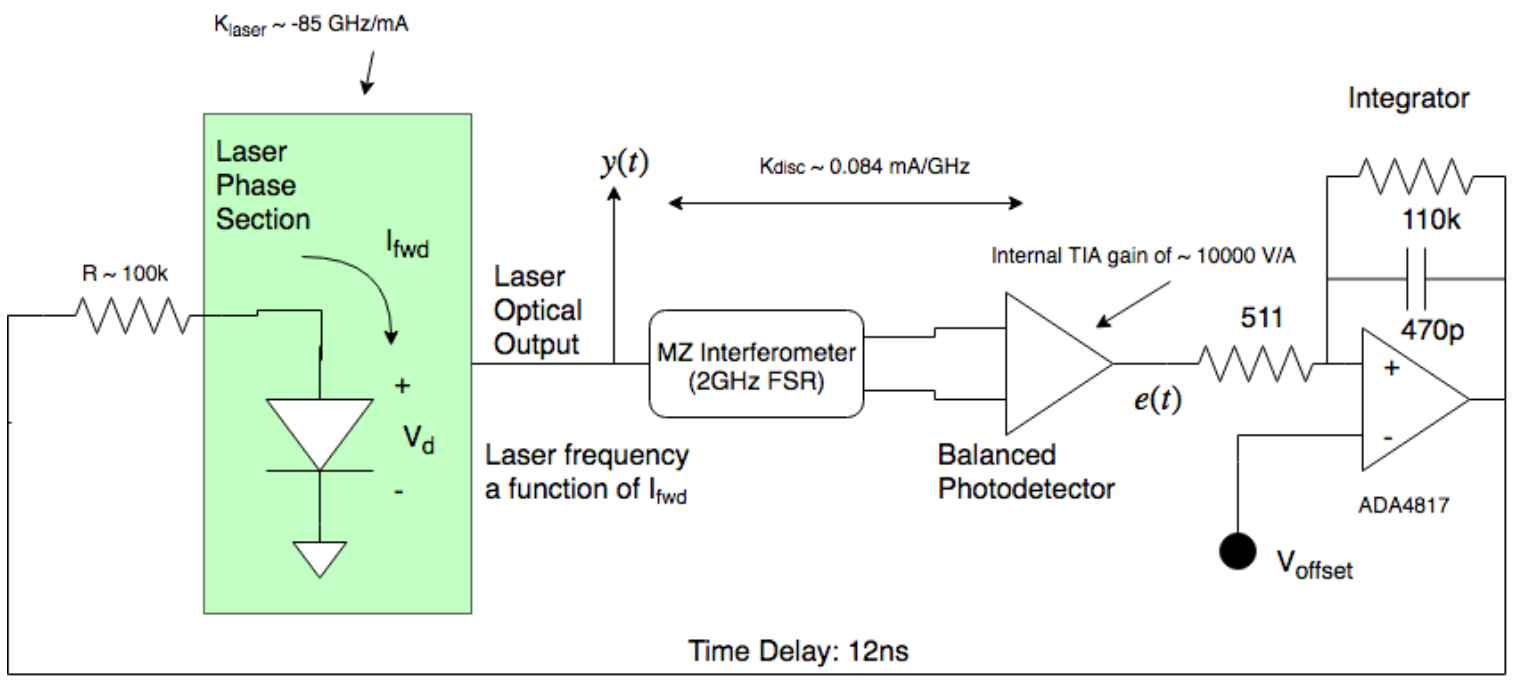

Figure 7.3: Block diagram of experimental OFLL with direct current injection into the phase section through a $100 \mathrm{k} \Omega$ resistor. The integrator was realized using an ADA4817 high-speed op-amp. 


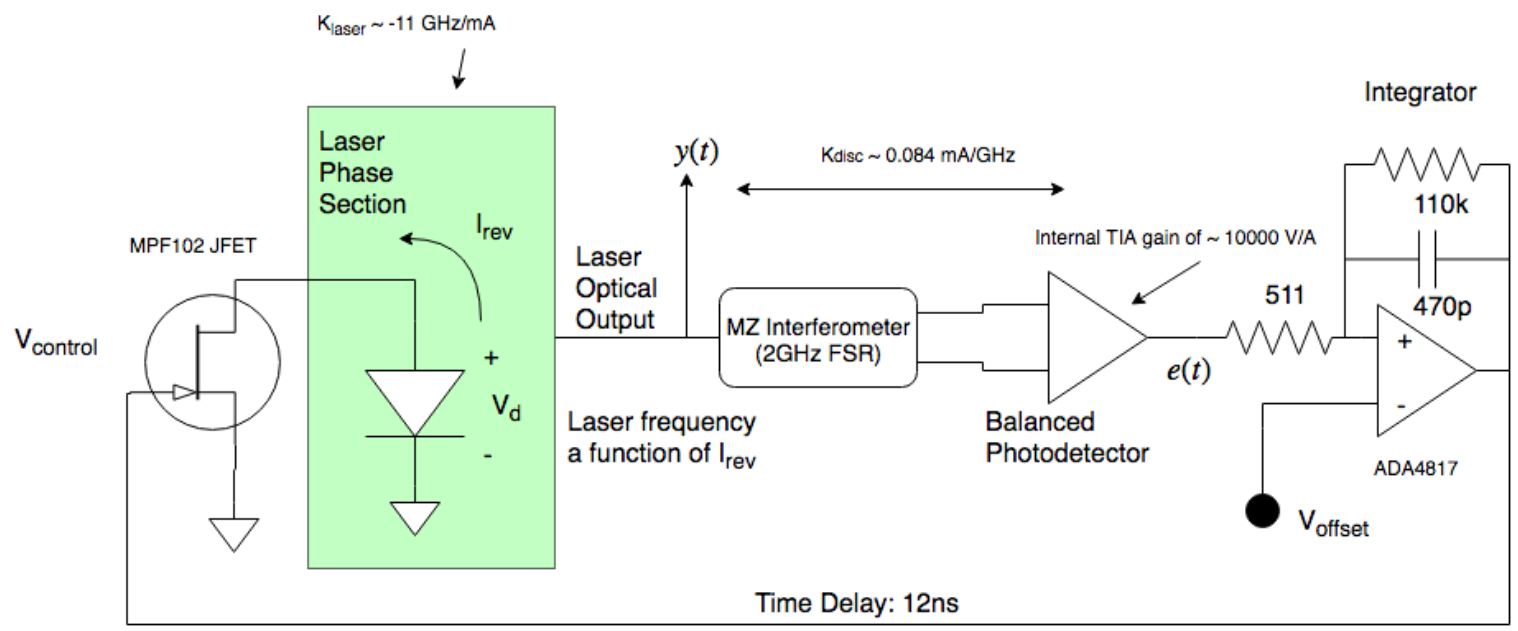

Figure 7.4: Block diagram of experimental OFLL with MPF102 JFET driver acting as a voltagecontrolled resistor performing resistance tuning on the laser phase section.

Two constants are used to convert changes in phase section current to a voltage signal $e(t)$, the phase section tuning constant $\mathrm{K}_{\text {laser }}[\mathrm{GHz} / \mathrm{mA}]$ and the discriminator constant $\mathrm{K}_{\mathrm{disc}}[\mathrm{mA} / \mathrm{GHz}]$. These constants were found in the previous chapter.

The system signal-to-noise ratio is determined by the amount of FM to AM conversion in the optical filter. This value can be found by comparing the spectrum of the RF output of the balanced detector when the slope of the frequency discriminator is zero (AM-only) and when it is a maximum at the quadrature position $(\mathrm{AM}+\mathrm{FM})$. The difference in these two signal levels in $\mathrm{dB}$ is the system SNR. The SNR was found to be more than $20 \mathrm{~dB}$ and can be seen in Figure 7.5. 


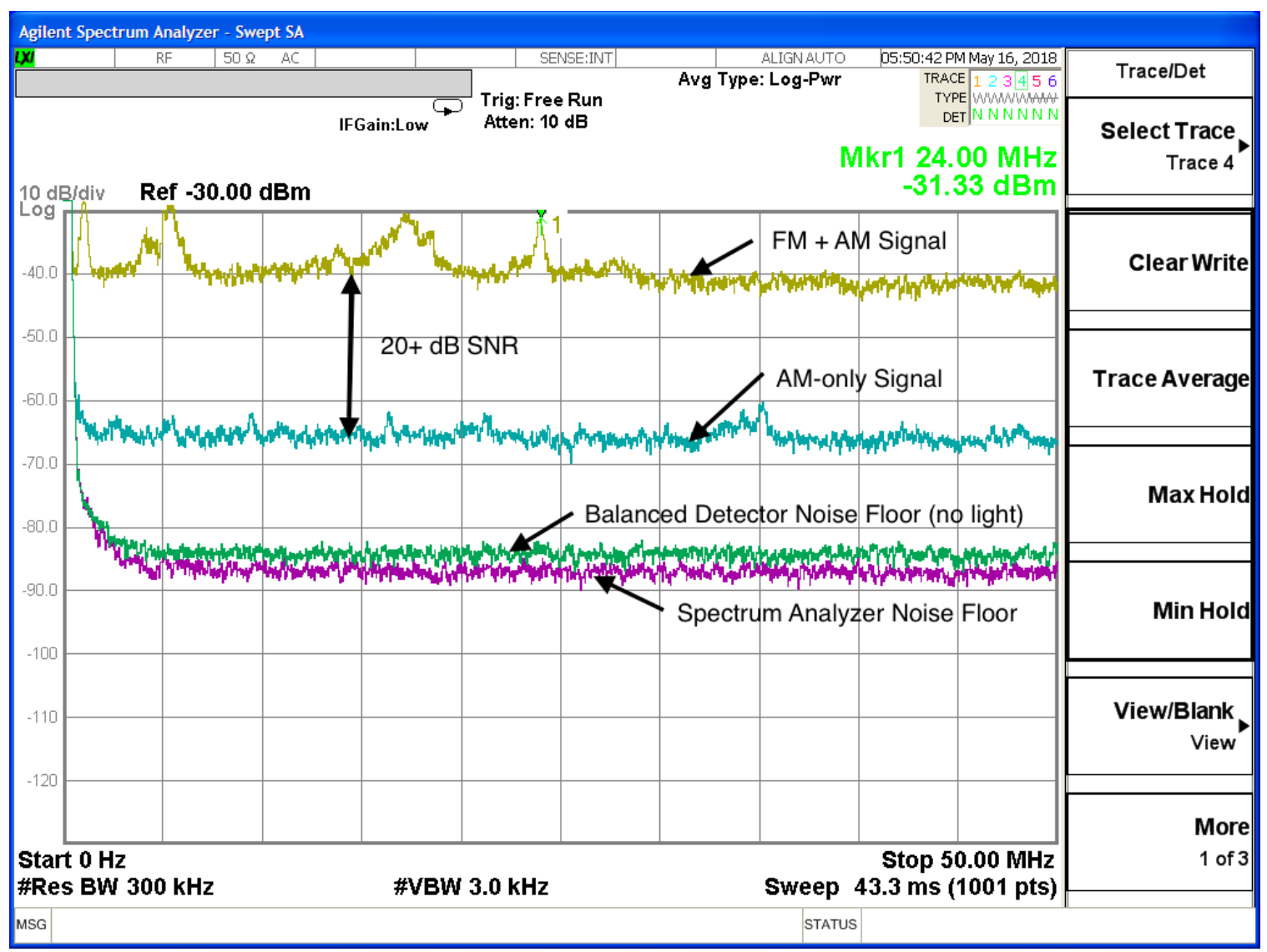

Figure 7.5: Determination of OFLL signal-to-noise ratio, given by the difference in dB of the FM + AM signal and the AM only signal. Both the AM only and FM + AM signals are well above the instrument noise floor.

It should be noted that the structure of the FM signal was not a constant with respect to bias conditions. There was a noticeable pick-up of local FM broadcast radio frequencies in the $80-110 \mathrm{MHz}$ range that was bias dependent. This pickup was likely due to battery connections to the board that inadvertently formed a loop antenna. Figure 7.6 shows two different bias conditions with different FM signal structures. 

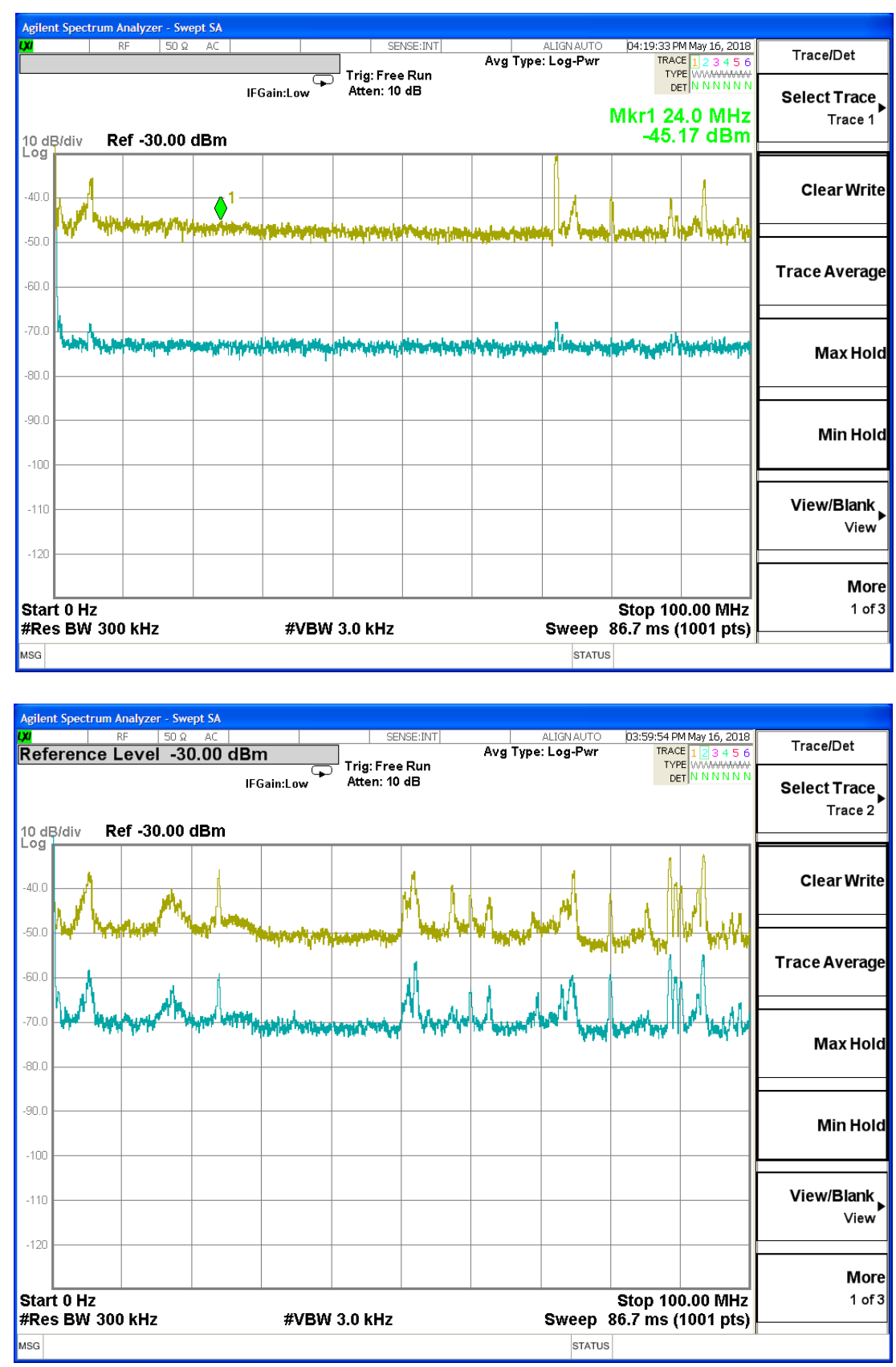

Figure 7.6: FM to AM conversion at two different bias conditions showing variation in FM noise spectrum. Both plots had $I_{\text {gain }} \approx 120 \mathrm{~mA}$ and $I_{\text {SOA }} \approx 30 \mathrm{~mA}$. Top plot was measured with $\mathbf{R}_{\mathrm{FM}}=\mathbf{R}_{\mathrm{BM}}=0 \Omega$ and the bottom plot was measured with $\mathbf{R}_{\mathrm{FM}}=0 \Omega$ and $\mathbf{R}_{\mathrm{BM}} \approx 5 \mathrm{k} \Omega$

\subsection{OFLL Simulation}

An open loop simulation of both the direct current injection and VCR tuned OFLL's was performed using LTspice to estimate each system's open loop DC gain and phase margin. The simulation assumes a linear relationship between both injected current vs. laser frequency as well as with frequency modulation on the signal into the optical filter vs. voltage out of the balanced detector. These linear 
relationships are defined by the constants $\mathrm{K}_{\text {laser }}$ and $\mathrm{K}_{\text {disc }}$. A lossless transmission line with a $50 \Omega$ characteristic impedance models the time delay due to overall loop length in both optical fiber and electrical conductor. Figure 7.7 shows the simulated frequency response of the integrator used in the OFLL.
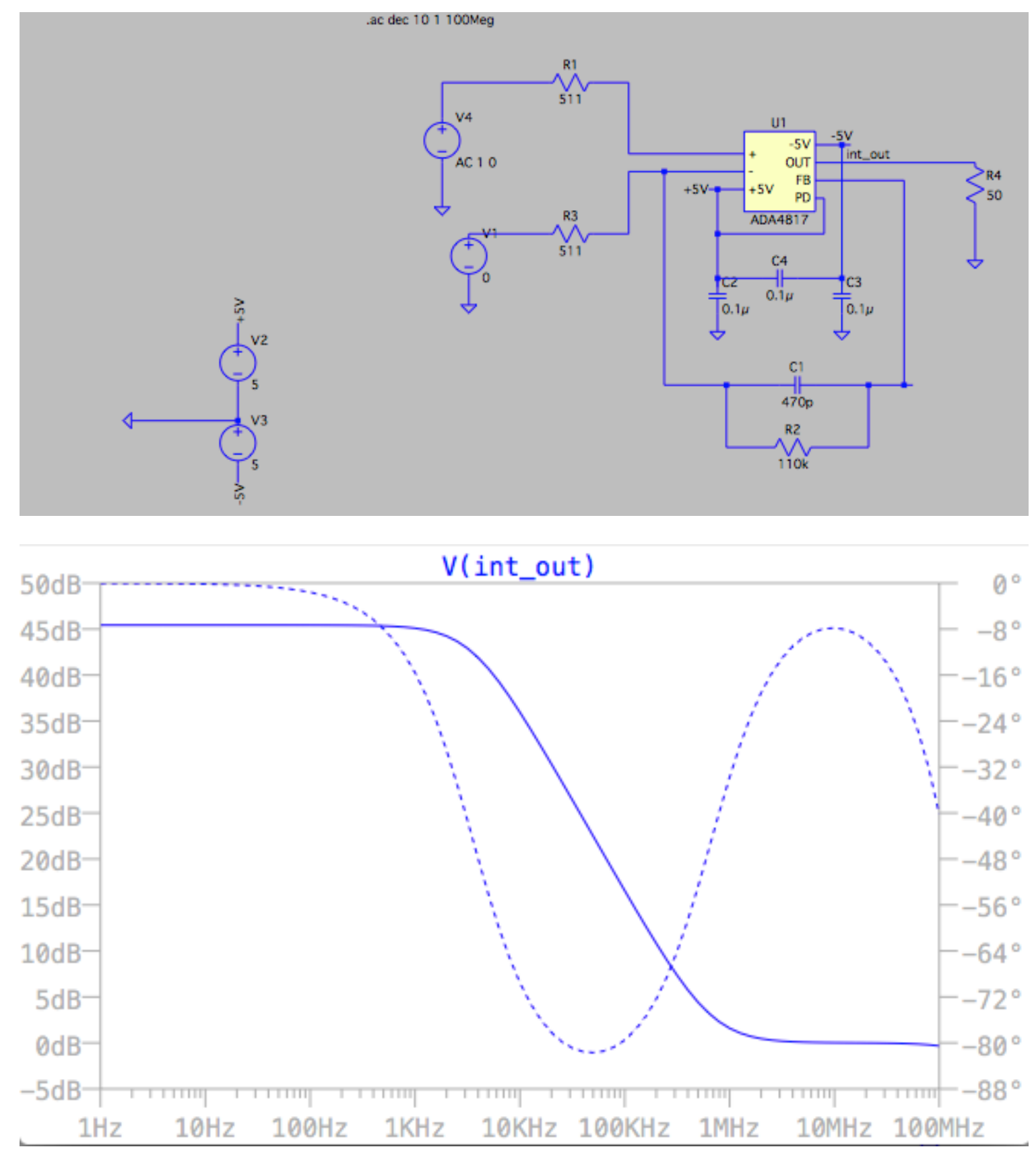

Figure 7.7: Simulated ADA4817 integrator schematic (top) and frequency response (bottom). The integrator input is an AC voltage source $\mathrm{V} 4$ and the output is the voltage across $\mathrm{R} 4$. The solid line shows the signal magnitude and the dashed line shows the phase shift. 
With all parts of the OFLL present, it is possible to do an open-loop simulation of the system. Figure 7.8 shows the simulation schematic for the direct injection case, and Figure 7.9 shows the simulated open-loop response of that system.

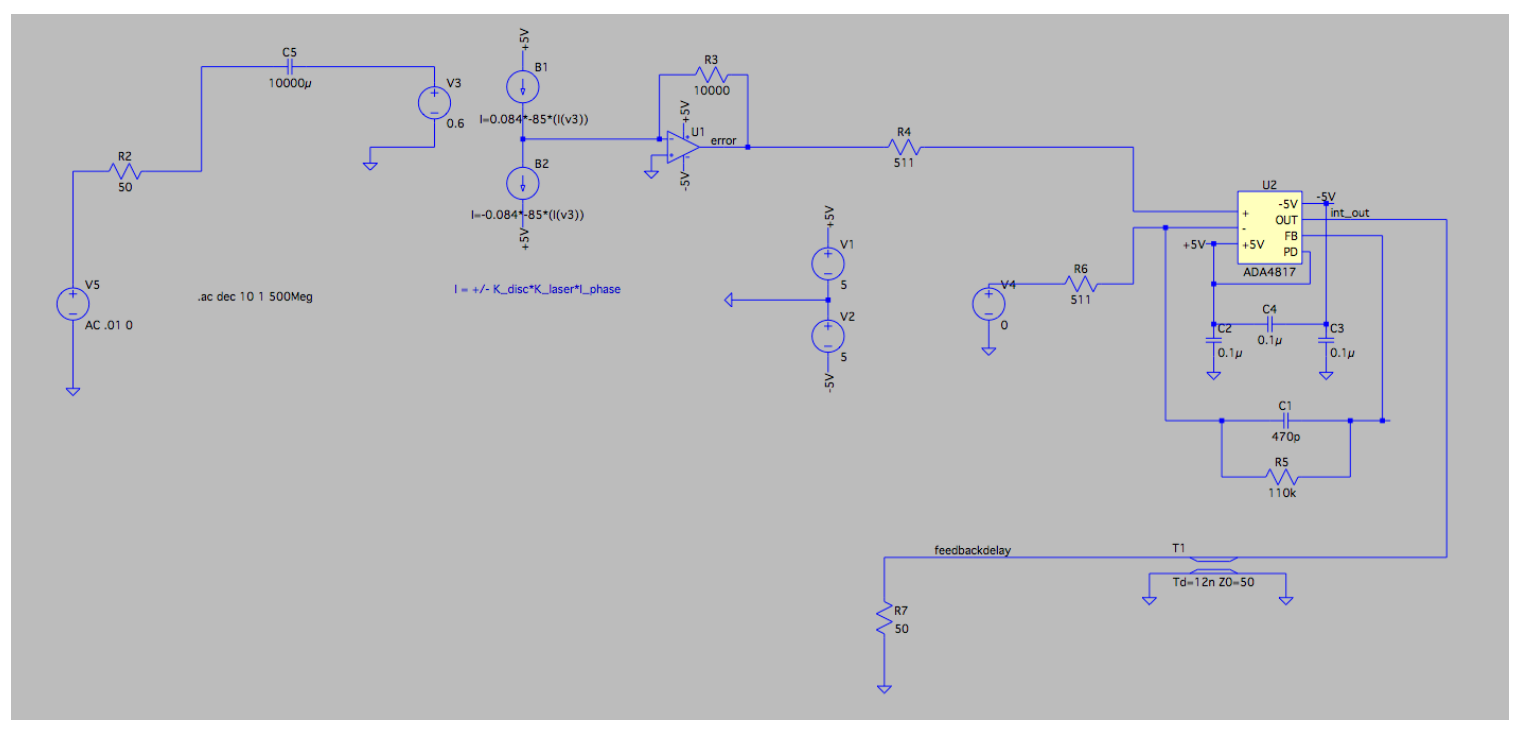

Figure 7.8: Open loop LTspice schematic of direct current injection OFLL. This simulation builds upon the simulation shown in the top plot of Figure 6.30 by routing the error signal out of the transimpedence amplifier through the ADA4817 integrator and a lossless $50 \Omega$ transmission line with a time delay given by the overall delay in the loop.

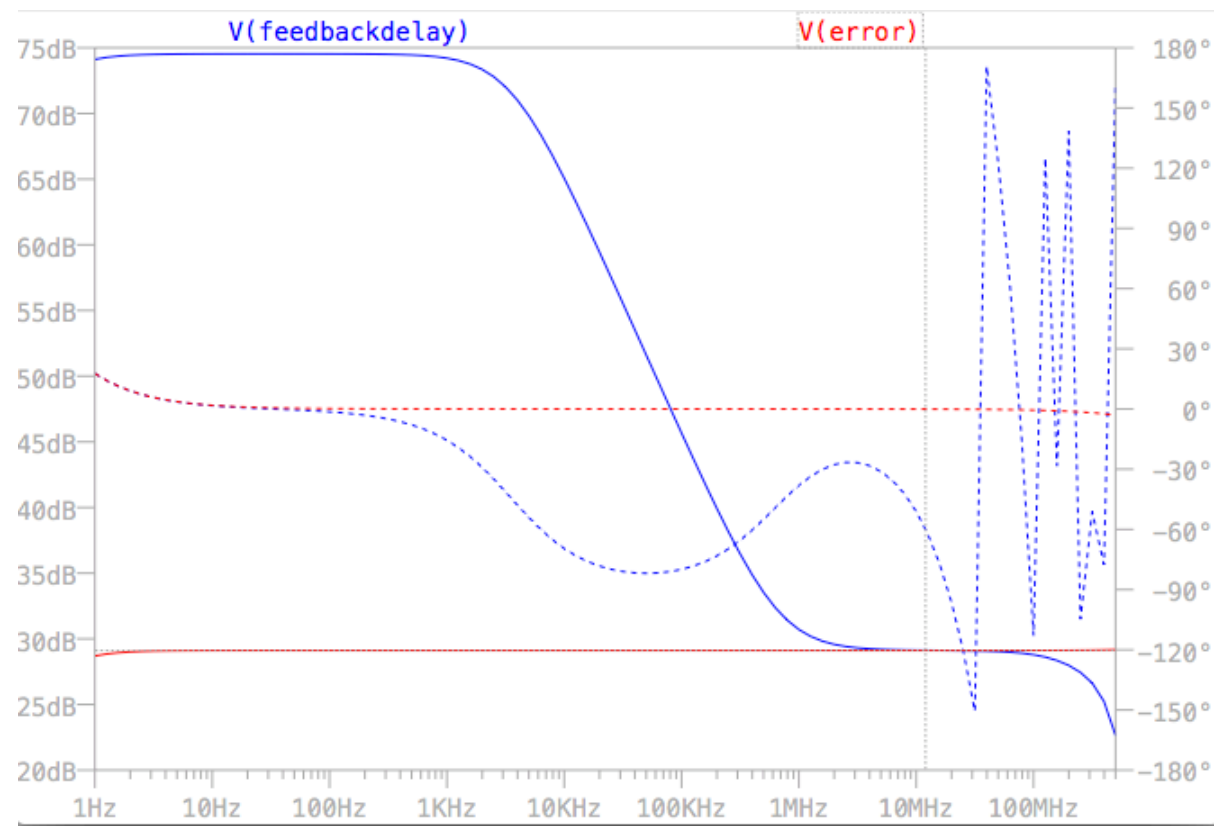

Figure 7.9: Simulated open loop frequency response of OFLL tuned via direct current injection. The solid line shows the signal magnitude and the dashed line shows the phase shift. The signal at the output of the transmission line is given by $\mathrm{V}$ (feedbackdelay) and can be seen to have a $75 \mathrm{~dB}$ gain at

DC and a $-3 \mathrm{~dB}$ bandwidth around $10 \mathrm{kHz}$. Phase margin is around $70^{\circ}$ at roughly $15 \mathrm{MHz}$. 
In Figure 7.8, the error signal is the signal out of the balanced detector, and "feedbackdelay" is the signal out of the integrator including the time delay of the system. The time delay was estimated to be $12 \mathrm{~ns}$ from about 1.5 meters of combined coax cable and optical fiber. The integrator has a DC gain of $45 \mathrm{~dB}$, and when driven by $50 \Omega$ the entire open-loop system with direct injection has a DC gain of $75 \mathrm{~dB}$. The phase margin of this system is around 70 degrees at a frequency $\mathrm{f}_{\mathrm{OdB}} \approx 15 \mathrm{MHz}$.

A similar open loop frequency response simulation was done for the OFLL with a JFET driver.

The LTspice schematic of this system can be seen in Figure 7.10 and the frequency response can be seen in Figure 7.11. The entire open-loop system has a DC gain of $20 \mathrm{~dB}$, with the error signal at $-25 \mathrm{~dB}$. The phase margin of this system is around 75 degrees at a frequency $\mathrm{f}_{0 \mathrm{~dB}} \approx 17 \mathrm{MHz}$.

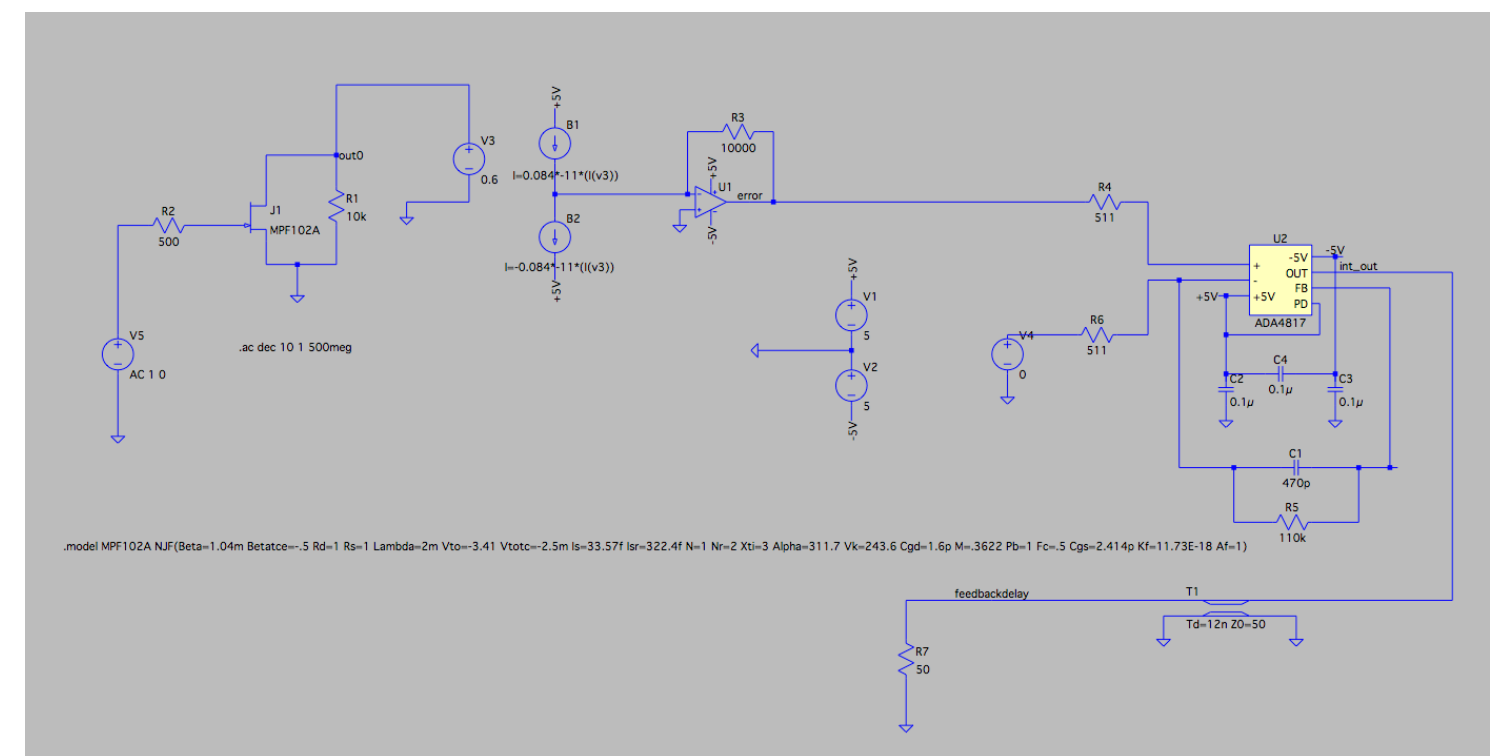

Figure 7.10: Open loop LTspice schematic of JFET driven OFLL. This simulation builds upon the simulation shown in the bottom plot of Figure 6.30 by routing the error signal out of the transimpedence amplifier through the ADA4817 integrator and a lossless $50 \Omega$ transmission line with a time delay given by the overall delay in the loop. 


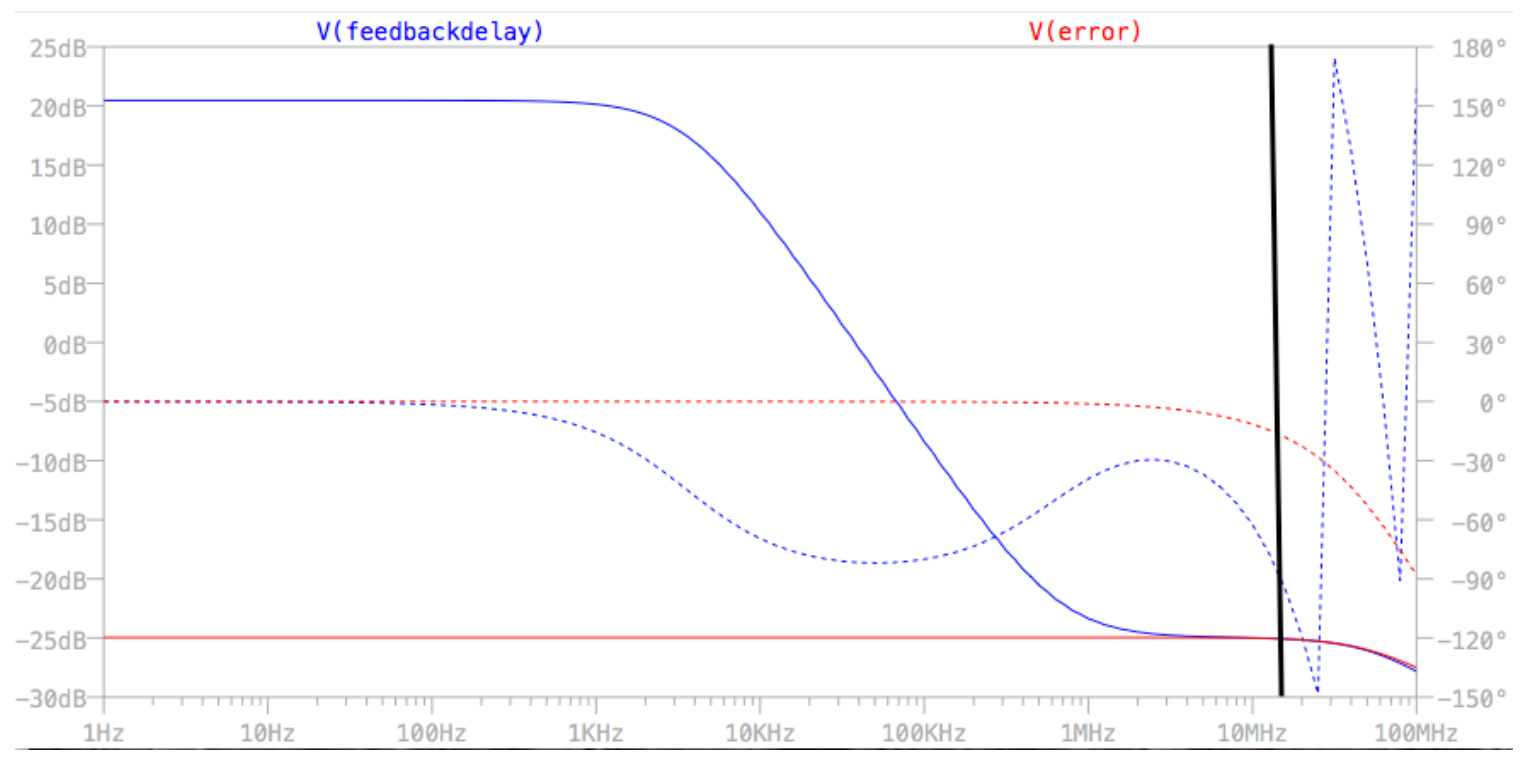

Figure 7.11: Simulated open loop frequency response of JFET driven OFLL. The solid line shows the signal magnitude and the dashed line shows the phase shift. The signal at the output of the transmission line is given by $\mathrm{V}$ (feedbackdelay) and can be seen to have a $20 \mathrm{~dB}$ gain at DC and a $-3 \mathrm{~dB}$ bandwidth around $10 \mathrm{kHz}$. Phase margin is around $75^{\circ}$ at roughly $17 \mathrm{MHz}$

\subsection{Open Loop and Closed Loop Performance}

The laser could be tuned into the locked state via the resistance tuning method of Section 6.4 on the mirror sections. For both the direct current injection and JFET driven OFLL, however, when the feedback loop was closed the system could only achieve DC locking. This means that the loop is able to maintain frequency but unable to reduce the laser phase noise and thus the laser linewidth. Due to time restraints and difficulty in further reducing the physical loop length, linewidth reduction was not yet achieved with the frequency- locked loop. This issue is potentially a result of the overall time delay in the loop or an insufficient amount of closed-loop gain. While this system may or may not be close to working, more work must be done to determine the root of the problem.

While both loop topologies can achieve DC locking, the OFLL with direct current injection showed a more robust locking state that covered a larger range of inputs. As a reference, the wavelength change given by resistance tuning of each mirror section in the unlocked state is shown in Figure 7.12 and the averaged unlocked linewidth is shown in Figure 7.13. 


\section{Back Mirror Tuning with Feedback Disabled}

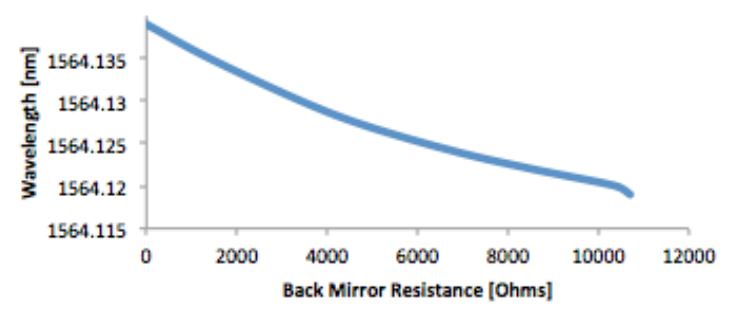

\section{Front Mirror Tuning with Feedback Disabled}

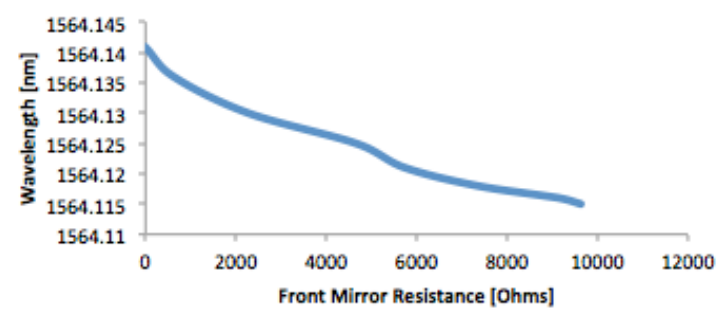

Figure 7.12: Resistance tuning of the mirror sections with feedback disabled. Each section shows a wavelength tuning range of about $30 \mathrm{pm}$. In this measurement $I_{\text {gain }} \approx 120 \mathrm{~mA}, I_{S O A} \approx 30 \mathrm{~mA}$, and the unused passive sections were shorted to ground

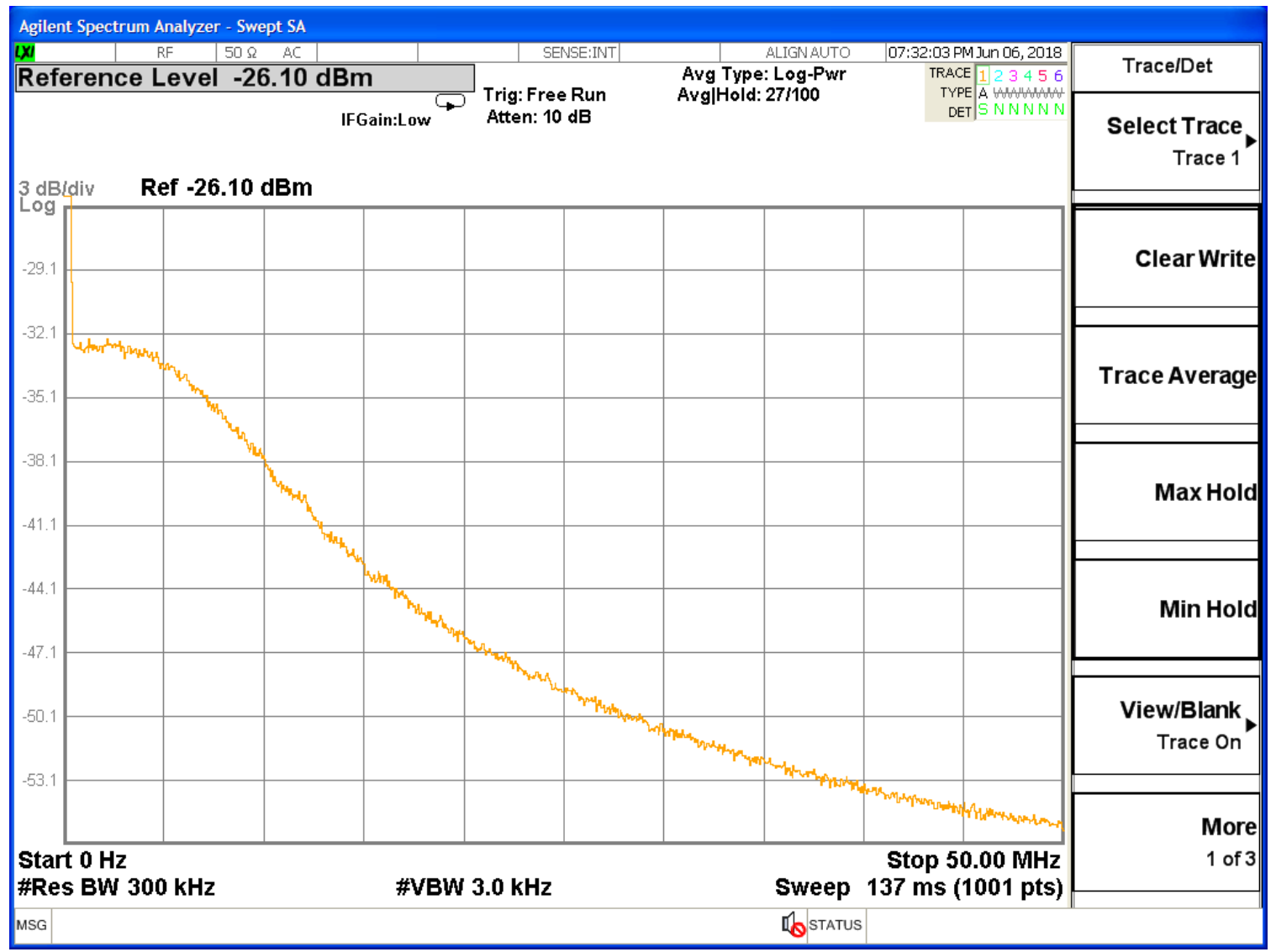

Figure 7.13: Self-Homodyne signal shows laser linewidth with feedback disabled around 7MHz. In this measurement $I_{\text {gain }} \approx 120 \mathrm{~mA}, I_{S O A} \approx 30 \mathrm{~mA}, R_{\mathrm{FM}}=0 \Omega$, and $R_{\mathrm{BM}}=10 \mathrm{k} \Omega$.

When feedback is enabled, the system can be brought into the locked state by adjusting the laser frequency to the optical filter quadrature position. Figures 7.14 and 7.15 show loop does indeed lock the laser frequency in the direct current injection and JFET driven OFLL's respectively. A binary lock state indicator is plotted along with the laser wavelength over the resistance tuning range. When the loop is 
locked, there is no change in laser wavelength for a change in resistance. The averaged self-homodyne signal for each topology is shown in Figures 7.16 and 7.17, showing no linewidth reduction in either case.

Back Mirror Tuning with Feedback Enabled: Direct Drive
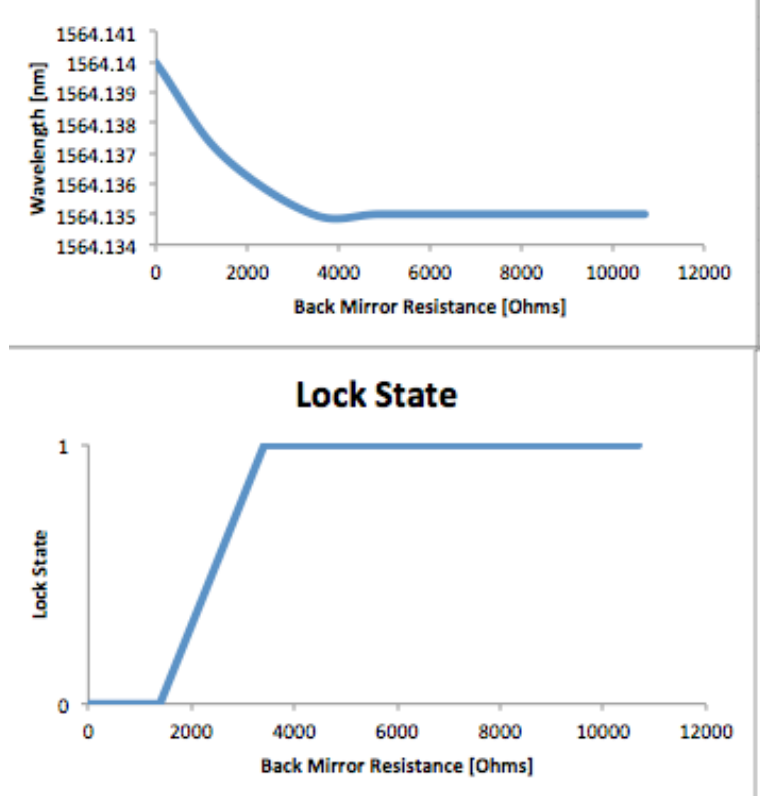

\section{Front Mirror Tuning with Feedback} Enabled: Direct Drive
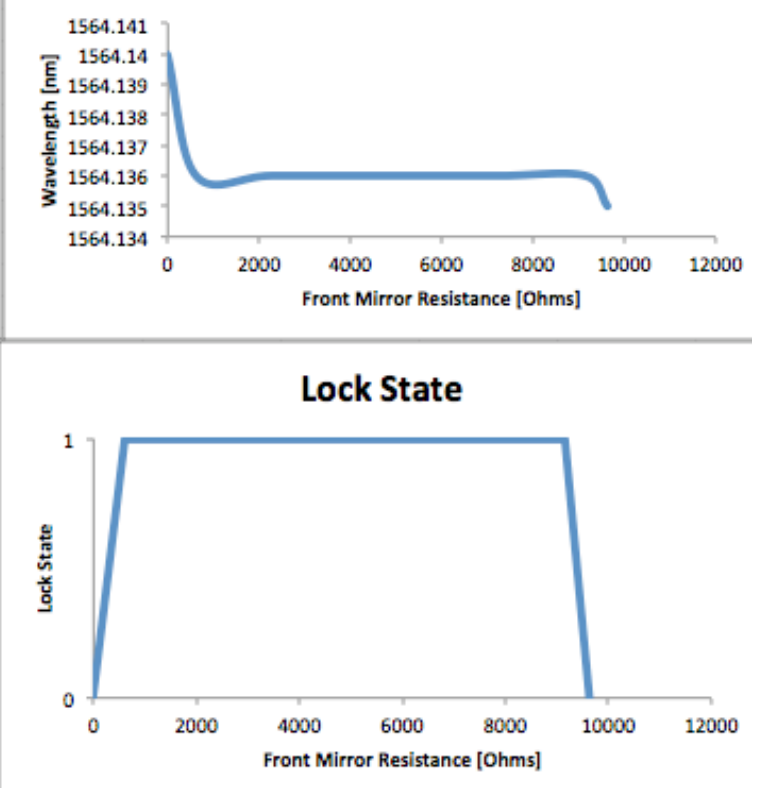

Figure 7.14: Resistance tuning of the mirror sections with feedback enabled in the direct current injection case. In this measurement $I_{\text {gain }} \approx 120 \mathrm{~mA}$ and $I_{S O A} \approx 30 \mathrm{~mA}$. The unused mirror section in each measurement was shorted to ground. When the loop is locked, there is no change in laser wavelength for a change in bias conditions within the locking range of the loop. Direct current injection OFLL is seen to have a more robust locking capability over a wider range of tuning resistance 

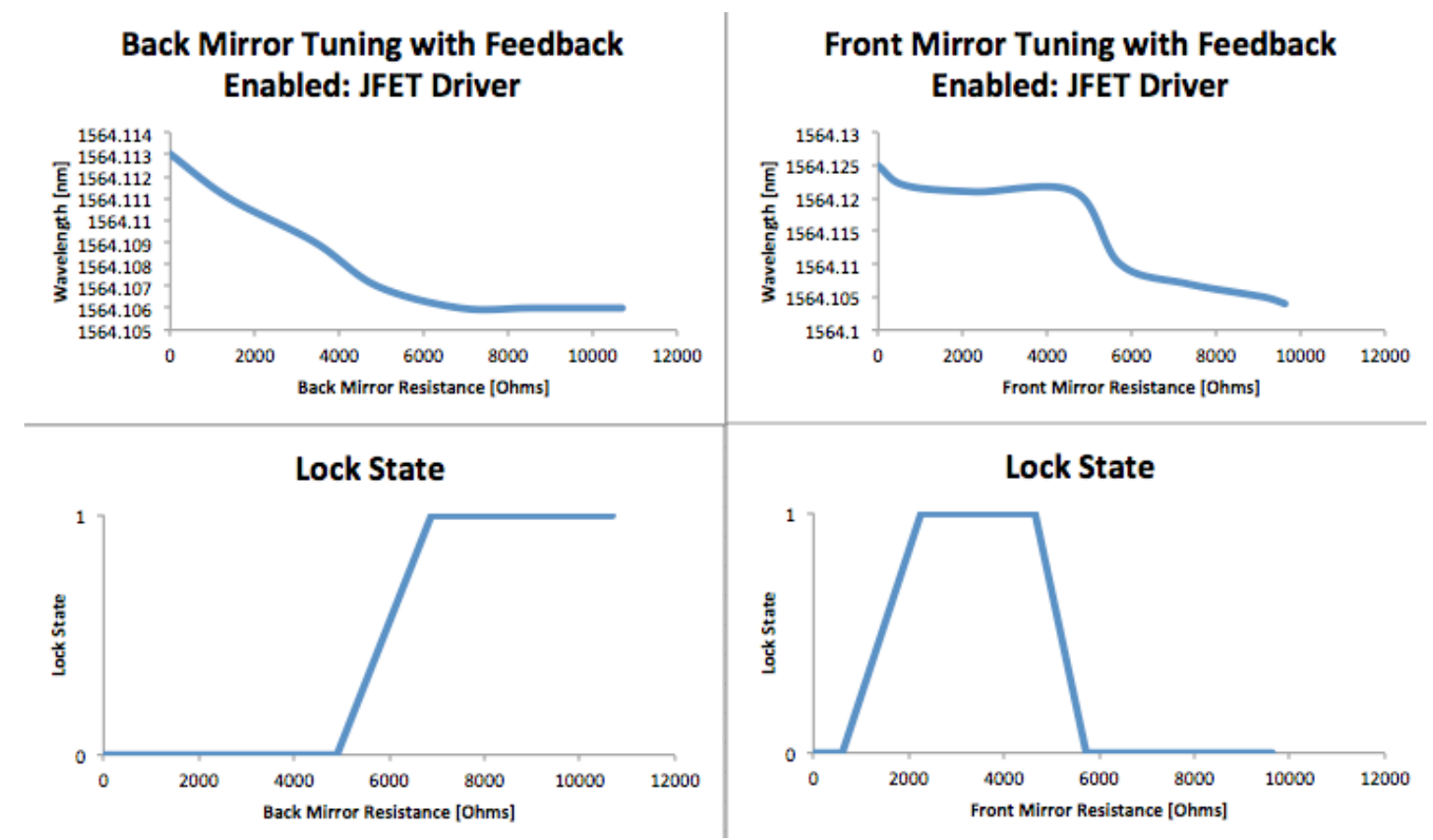

Figure 7.15: Resistance tuning of the mirror sections with feedback enabled in the JFET driven case. In this measurement $I_{\text {gain }} \approx 120 \mathrm{~mA}$ and $I_{S O A} \approx 30 \mathrm{~mA}$. The unused mirror section in each measurement was shorted to ground. When the loop is locked, there is no change in laser wavelength for a change in bias conditions within the locking range of the loop. Smaller locking range in the JFET driven OFLL shows a less robust DC lock compared to the direct current injection case. 


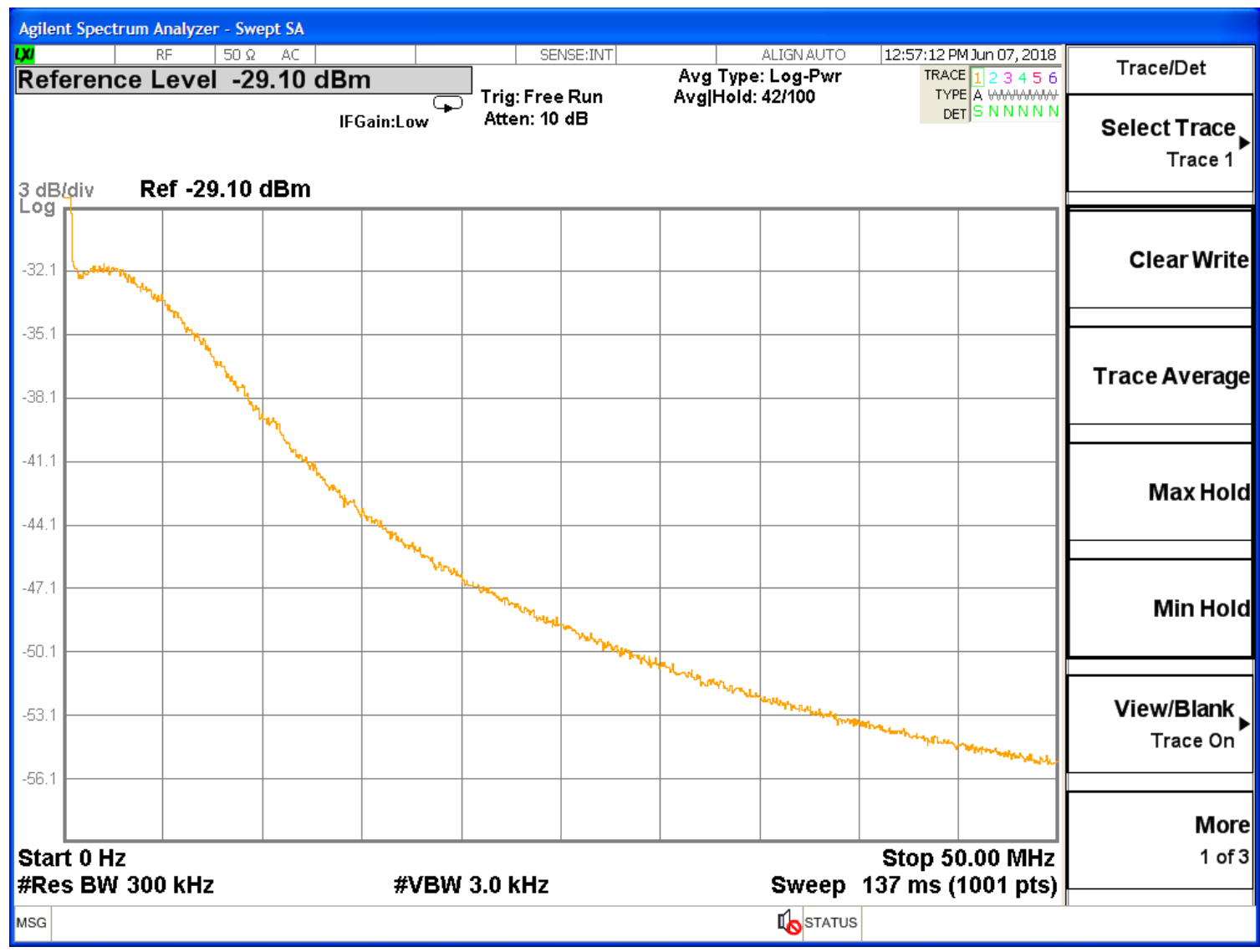

Figure 7.16: Self-Homodyne signal for OFLL with direct current injection with feedback enabled shows laser linewidth around $7 \mathrm{MHz}$. In this measurement $I_{\text {gain }} \approx 120 \mathrm{~mA}, I_{S O A} \approx 30 \mathrm{~mA}, R_{\mathrm{FM}}=0 \Omega$, and $R_{B M}=10 \mathrm{k} \Omega$. In this case, the mirror sections are under reverse bias while the phase section is under forward bias. 


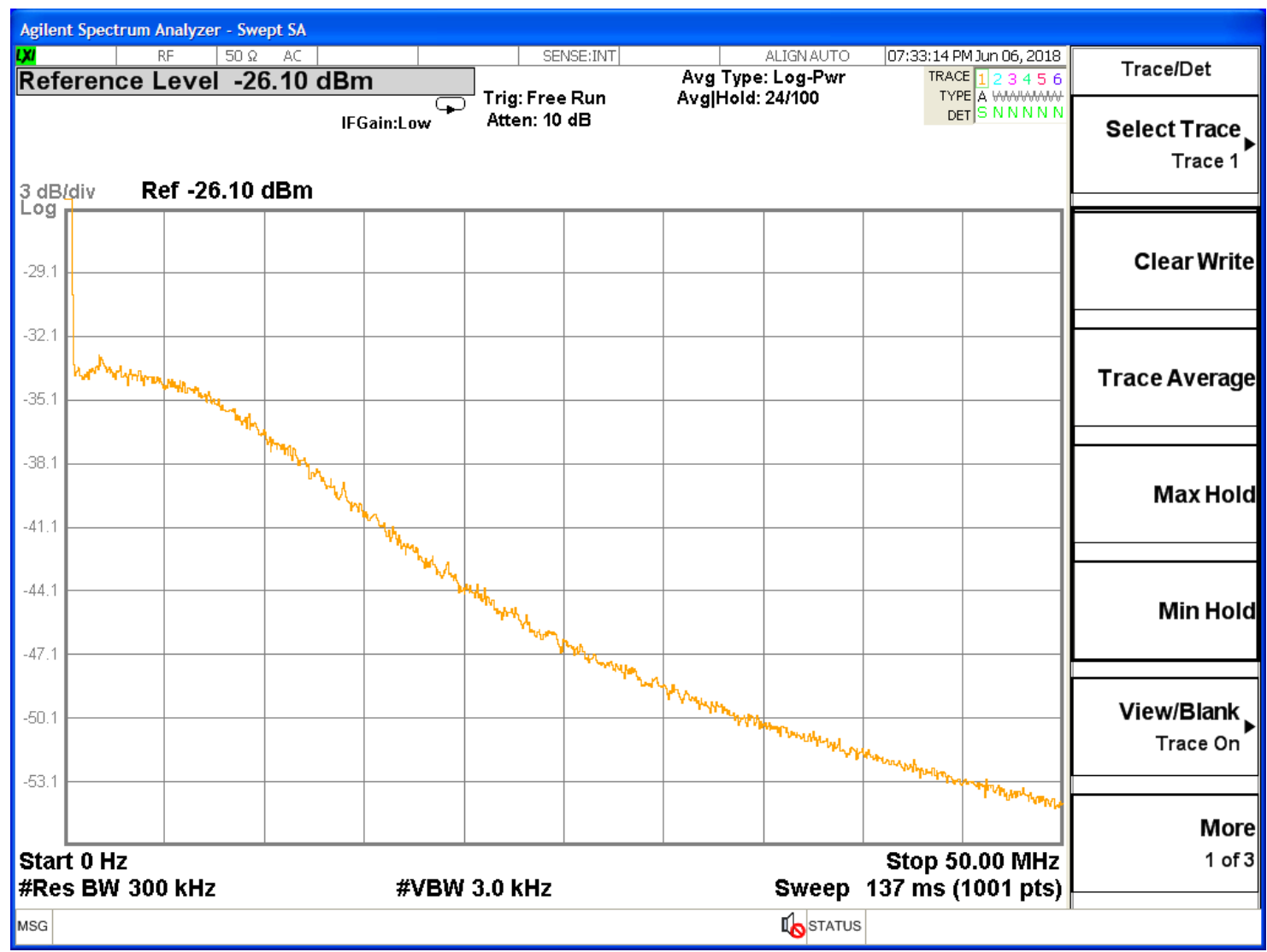

Figure 7.17: Self-Homodyne signal for OFLL with JFET driver with feedback enabled shows laser linewidth around $10 \mathrm{MHz}$. In this measurement $I_{\text {gain }} \approx 120 \mathrm{~mA}, I_{S O A} \approx 30 \mathrm{~mA}, R_{\mathrm{FM}}=0 \Omega$, and $R_{B M}=10 \mathrm{k} \Omega$. In this case, the phase section and mirror sections are all under reverse bias. 


\section{Chapter 8: Conclusions and Future Directions}

In conclusion, the reverse bias mode of operation (Figure 3.10, Region C) was found to be far better suited for LIDAR than the forward bias mode of operation (Figure 3.10, Regions A \& B). Both the external reverse bias tuning and resistance tuning methods can be used to produce FMCW sweeps with a bandwidth greater than $7 \mathrm{GHz}$. A summary of the results found in this work are shown in Table 8.1, which shows the sweep bandwidth, linewidth, range resolution, and coherence length for each mode of VT-DBR operation.

\begin{tabular}{|c|c|c|c|c|c|}
\hline Mode of Operation & $\begin{array}{l}\text { Sweep with narrow } \\
\text { linewidth? (B) }\end{array}$ & Linewidth & $\begin{array}{l}\text { Range Resolution } \\
\mathrm{dR}=\mathrm{C} / 2 \mathrm{~B}\end{array}$ & $\begin{array}{l}\text { Range Estimate } \\
\text { (Coherence } \\
\text { Length) }\end{array}$ & Comments \\
\hline $\begin{array}{l}\text { Full Scale Forward } \\
\text { Bias (Region A) }\end{array}$ & $\begin{array}{l}\text { not found, potentially with } \\
\text { finer mapping of } \\
\text { wavelength and linewidth } \\
\text { vs. tuning currents }\end{array}$ & $\begin{array}{l}>100 \mathrm{MHZ} \\
\text { for most bias } \\
\text { conditions }\end{array}$ & $n / a$ & $<1.3$ meters & $\begin{array}{l}\text { Linewidth too wide for LIDAR } \\
\text { at most bias conditions }\end{array}$ \\
\hline $\begin{array}{l}\text { Small Current } \\
\text { Forward Bias } \\
\text { (Region B) }\end{array}$ & $\begin{array}{l}\text { not found, potentially with } \\
\text { finer mapping of } \\
\text { wavelength and linewidth } \\
\text { vs. tuning currents }\end{array}$ & $\begin{array}{l}>100 \mathrm{MHZ} \\
\text { for most bias } \\
\text { conditions, small } \\
\text { regions of } \\
\text { narrow } \\
\text { linewidth }\end{array}$ & $\mathrm{n} / \mathrm{a}$ & $<1.3$ meters & $\begin{array}{l}\text { Linewidth too wide for LIDAR } \\
\text { at most bias conditions. Small } \\
\text { regions of narrow linewidth } \\
\text { exist, but tuning paths are far } \\
\text { less robust }\end{array}$ \\
\hline $\begin{array}{l}\text { Reverse Current } \\
\text { Bias } \\
\text { (Region C) }\end{array}$ & $\sim 7 \mathrm{GHz}$ & $2 \mathrm{MHz}$ & 2.2 centimeters & 44 meters & $\begin{array}{l}\text { Promising for LIDAR due to } \\
\text { robust tuning paths with } \\
\text { narrow linewidth }\end{array}$ \\
\hline $\begin{array}{l}\text { Resistance Tuning } \\
\text { (Region C) }\end{array}$ & $\sim 10 \mathrm{GHz}$ & $2 \mathrm{MHz}$ & 1.8 centimeters & 44 meters & $\begin{array}{l}\text { Promising for LIDAR due to } \\
\text { robust tuning paths with } \\
\text { narrow linewidth }\end{array}$ \\
\hline
\end{tabular}

Table 8.1: Summary of LIDAR potential for each mode of VT-DBR operation

Table 8.1 shows that the photo-detector mode of operation in Region $\mathrm{C}$ is better suited for LIDAR than the forward current injection modes. The small current forward biasing in Region B could also potentially be used for LIDAR, but it would require a finer mapping of the wavelength and linewidth space in Region B to determine if there are tuning paths with linewidth narrow enough to be useful for LIDAR.

Also promising is the possibility of FMCW sweeps with a linewidth reduced via OFLL. With more work it should be possible to achieve AC locking of the OFLL and significantly reduce laser phase noise. If the OFLL can be successfully implemented over a range of frequencies, this method of linewidth reduction would drastically improve VT-DBR laser performance in LIDAR applications. If the linewidth could be reduced to say $100 \mathrm{kHz}$, the maximum range limit would be well in excess of a kilometer. Paired with a $10 \mathrm{GHz}$ tuning range giving a range resolution of $1.5 \mathrm{~cm}$, this leads to a ratio of range resolution to 
range on order of $10^{-5}$. This high performance LIDAR system would be ideal for imaging in autonomous vehicles such as self-driving cars or military UAVs.

In comparison, using the resistance tuning method without any linewidth reduction would lead to more than $7 \mathrm{GHz}$ of tuning with $2-4 \mathrm{MHz}$ linewidth. A LIDAR system with these characteristics would be limited to around $50 \mathrm{~m}$ with a $2 \mathrm{~cm}$ range resolution. These numbers give a range resolution to range ratio of .0004 , which is still adequate for a number of LIDAR applications such as industrial machine vision, meteorology, and facial recognition.

There are many possible future directions of this project. The first obvious direction is to continue troubleshooting the OFLL to achieve AC locking and linewidth reduction. If linewidth reduction can be achieved, the system must still be adapted to be able to sweep laser frequency with a reduced linewidth. Secondly, the process to measure wavelength and linewidth could be automated to produce maps of each mode of operation with a much finer resolution. This fine resolution would make it possible to search for low-linewidth sweeps in regions A and B. Finally, a third possible direction would be to analyze VT-DBR performance in region $\mathrm{C}$ with a combination of external reverse bias and resistance tuning. Resistance tuning of VT-DBR passive sections biased as photo-detectors could lead to an increased tuning range with narrow linewidth, further increasing the utility of this mode of operation for LIDAR applications. 


\section{Bibliography}

[1] Albert Jelalian, Laser Radar Systems, Norwood, Massachusetts: Artech House, 1992.

[2] Analog Devices, “ADA4817-1/ADA4817-2,” 23 April 2018,

$<$ http://www.analog.com/media/en/technical-documentation/data-sheets/ada4817-

1_4817-2.pdf>

[3] Arseny Vasilyev, "The Optoelectronic Swept-Frequency Laser and Its Applications in Ranging, Three-Dimensional Imaging, and Coherent Beam Combining of Chirped-Seed Amplifiers" California Institute of Technology, Pasadena, California, May 2013.

[4] Brandon George, "Swept-Frequency Sampled Grating Distributed Bragg Reflector Lasers Optimized for Optical Coherence Tomography Applications" Electrical Engineering Department, California Polytechnic State University, San Luis Obispo, Dec 2009.

[5] Brett Browning, Jean-Emmanuel Deschaud, David Prasser, and Peter Rander, "3D Mapping for high-fidelity unmanned ground vehicle lidar simulation"

[6] C. J. Karlsson, F. Å. A. Olsson, D. Letalick, and M. Harris, “All-fiber multifunction continuous-wave coherent laser radar at 1.55 um for range, speed, vibration, and wind measurements," Appl. Opt. 39(21), 3716-3726, (2000).

[7] David Walsh, "Warfighters reap benefits of LIDAR mapping technology" 25 April 2018, $<$ https://defensesystems.com/Articles/2011/07/18/Tech-Watch-GEOINT-

LIDAR.asp $x$ ?Page $=1>$

[8] Dennis Derickson, Fiber Optic Test and Measurement, Upper Saddle River, New Jersey: Prentice Hall, 1998.

[9] Dennis Derickson, Mike Bernacil, Andrew DeKelaita, Ben Maher, Shane O'Connor, Matthew N. Sysak, and Leif Johanssen, "SGDBR single-chip wavelength tunable lasers for swept source OCT," Coherence Domain Optical Methods and Optical Coherence Tomography in Biomedicine XII Proceedings, Volume 6847, (2008).

[10] Diego Pierrottet, Farzin Amzajerdian, Larry Petway, Bruce Barnes, George Lockard, and Manuel Rubio, "Linear FMCW Laser Radar for Precision Range and Vector Velocity Measurements" Material Research Society (MRS) 2008 Spring Meeting; 14-18 Mar. 2008; San Francisco, CA; United States

[11] Eblana Photonics, "Linewidth Measurment," 23 April 2018, $<$ http://www.eblanaphotonics.com/downloads/Linewidth.pdf>

[12] Eric Moore, "Advances in Swept-Wavelength Interferometry for Precision Measurements," Department of Electrical, Computer, and Energy Engineering, University of Colorado, 2011.

[13] Erke Shang, Xiangjing An, Tao Wu, Tingbo Hu, Qiping Yuan, and Hangen He, "LiDAR Based Negative Obstacle Detection for Field Autonomous Land Vehicles" Journal of Field Robotics 33(5), 591-617 (2016)

[14] Farhan Rana, "Basics of Semiconductor Lasers" 12 April 2018, $<$ https://courses.cit.cornell.edu/ece533/Lectures/handout11.pdf $>$ 
[15] Finisar, "Product Specification CW Tunable Laser - Butterfly Package S7500," 23 April 2018, <https://www.finisar.com/sites/default/files/downloads/finisar_s7500_cw_tunable_ laser_butterfly_package_product_specification.pdf>

[16] Frank Famose, "Assessment of the use of spectral domain optical coherence tomography (SD-OCT) for evaluation of the healthy and pathological cornea in dogs and cats," Veterinary Ophthalmology, Vol.17, pp12-22 (2014)

[17] G. Sarlet, G. Morthier and R. Baets, "Control of widely tunable SSG-DBR lasers for dense wavelength division multiplexing, " Journal of Lightwave Technology, vol. 18, no. 8, pp. 1128-1138, Aug. 2000.

[18] Insight Photonic Solutions, 1 April 2018, <http://www.sweptlaser.com>

[19] Joseph C. Palais, Fiber Optic Communications, Fifth Edition, New Jersey: Pearson Prentice Hall, 2005.

[20] Kai Shi, Frank Smyth, Prince M. Anandarajah, Douglas Reid, Yonglin Yu, and Liam P. Barry, "Linewidth of SG-DBR laser and its effect on DPSK transmission" Optics Communications, Vol. 283, pp5040-5045, (2010)

[21] M. Harris, G. N. Pearson, J. M. Vaughan, D. Letalick \& C. Karlsson (1998) The role of laser coherence length in continuous-wave coherent laser radar, Journal of Modern Optics, 45:8, 1567-1581, DOI: 10.1080/09500349808230653

[22] M.C. Amann and Thierry Bosch. "Laser ranging: A critical review of unusual techniques for distance measurement," Optical Engineering, 40(1), 10. (2001).

[23] M.C. Amann, R. Hakimi, B. Borchert, and S. Illek. "Linewidth Broadening by $1 / \mathrm{f}$ Noise in Wavelength-Tunable Laser Diodes," Appl. Phys. Lett. 70, 1512 (1997)

[24] M.C. Amann, "Phase Noise Limited Resolution of Coherent LIDAR Using Widely Tunable Laser Diodes" Electronics Letters, Vol 28, No. 78, Aug. 1992.

[25] M.C. Amann and Jens Buus, Tunable Laser Diodes, Norwood, Massachusetts: Artech House, 1998.

[26] Michael Minneman, Jason Ensher, Michael Crawford, and Dennis Derickson “AllSemiconductor High-Speed Akinetic Swept-Source for OCT” SPIE-OSA-IEEE, Vol. 8311, 2011.

[27] M. Musa and S. Salous, "Ambiguity elimination in HF FMCW radar systems" IEE Proc.Radaa Sonar Navig., Vol. 147, No. 4, August 2000.

[28] NOAA, "LIDAR - Light Detection and Ranging — is a remote sensing method used to examine the surface of the Earth," 24 April 2018, <https://www.webcitation.org/ 6H82i1Gfx?url=http://oceanservice.noaa.gov/facts/lidar.html> 
[29] Oliver Reitebuch, Christian Werner, Ines Leike, Patricia Delville, Pierre Flamant, Alexander Cress, and Dirk Engelbart, "Experimental Validation of Wind Profiling Performed by the Airborne 10-micrometer Heterodyne Doppler Lidar WIND.” Journal of Atmospheric \& Oceanic Technology, August 2001.

[30] ON Semiconductor, "MPF102", 23 April 2018, $<$ http://www.onsemi.com/pub/Collateral/MPF102-D.PDF>

[31] R. Christian Martens Biersach, Dennis Derickson, Jason Ensher, "Frequency Sweep Jitter and Wander of a Vernier-Tuned Distributed Bragg Reflector (VT-DBR) Laser at 1550 $\mathrm{nm}$ in OCT Applications" Optical Coherence Imaging Techniques and Imaging in Scattering Media, SPIE Proceedings (Optical Society of America, 2015)

[32] Roric Christian Martens Biersach, "Sweep Stability Characterization of a Vernier-Tuned Distributed Bragg Reflector (VT-DBR) All-Semiconductor Tunable Swept Laser System at $1550 \mathrm{~nm}$ for Sensing Applications" Electrical Engineering Department, California Polytechnic State University, San Luis Obispo, March 2015

[33] Sebastian Marschall, Birgit Sander, Mette Mogensen, Thomas M. Jørgensen, and Peter E. Andersen, "Optical coherence tomography — current technology and applications in clinical and biomedical research" Anal. Bioanal. Chem. 400:2699-2720, (2011)

[34] Shane O'Connor, "High Speed Wavelength Tuning of SG-DBR Lasers for Light Detection and Ranging and Optical Coherence Tomography," Electrical Engineering Department, California Polytechnic State University, San Luis Obispo, June 2008.

[35] Shaozhen Song, Jingjiang Xu, and Ruikang K. Wang, "Long-range and wide field of view optical coherence tomography for in vivo 3D imaging of large volume object based on akinetic programmable swept source" Biomedical Optics Express, Vol. 7, No. 11, Nov 2016

[36] Thorlabs, "OCT Selection Guide," 23 April 2018, $<$ https://www.thorlabs.com/newgrouppage9.cfm?objectgroup_id=5702>

[37] Thorlabs, "PDB100 Series Balanced Amplified Photodetectors," 23 April 2018, $<$ http://www.hisky-hk.com/images/200712/1198751136PDF56018.pdf> 